Draft version September 30, 2019

Preprint typeset using $\mathrm{LAT}_{\mathrm{E}} \mathrm{X}$ style emulateapj v. 08/22/09

\title{
A SEARCH FOR INTRINSIC HI 21 CM AND OH 18 CM ABSORPTION TOWARD COMPACT RADIO SOURCES
}

\author{
Kathryn Grasha ${ }^{1,2,3}$, Jeremy Darling ${ }^{3}$, Alberto Bolatto ${ }^{4}$, Adam K. Leroy ${ }^{5}$, John T. Stocke ${ }^{3}$ \\ Draft version September 30, 2019
}

\begin{abstract}
We present the results of a large search for intrinsic HI $21 \mathrm{~cm}$ and $\mathrm{OH} 18 \mathrm{~cm}$ absorption in 145 compact radio sources in the redshift range $0.02<z<3.8$ with the Green Bank Telescope. We re-detect HI $21 \mathrm{~cm}$ absorption toward six known absorption systems but detect no new $\mathrm{HI}$ or $\mathrm{OH}$ absorption in 102 interference-free sources. 79 sources have not previously been observed for $\mathrm{HI} 21 \mathrm{~cm}$ absorption. We recover a mean optical depth limit of $\tau_{3 \sigma}<0.023$ for all the non-detections in the survey. Our results do not support the high intrinsic absorption rates found by previous studies in compact radio sources at low redshift. Our results do, however, support the hypothesis proposed by Curran et al. (2008) that high ultraviolet (UV) luminosity active galactic nuclei (AGN) do not show intrinsic HI $21 \mathrm{~cm}$ absorption, confirming a threshold of $L_{\mathrm{UV}}=10^{23} \mathrm{~W} \mathrm{~Hz}^{-1}$, above which our intrinsic absorption fraction is zero (54 sources). The exact nature of the UV luminosity effect on HI absorption systems remains ambiguous. We additionally find no statistical correlation between the $1.4 \mathrm{GHz}$ radio luminosity or the source size and the $21 \mathrm{~cm}$ absorption detection rate. We attribute the lack of intrinsic absorption in our survey to the UV luminosity effect caused by an optical selection bias and a decreased column density sensitivity with increasing redshift due to lower radio continuum flux densities, high radio frequency interference, and higher telescope system temperatures at low frequencies.
\end{abstract}

Subject headings: radio lines: galaxies — quasars: absorption lines — galaxies: nuclei — galaxies: ISM — galaxies: active - galaxies: general

\section{INTRODUCTION}

Many compact radio sources are likely to be young and/or confined radio jets. It is likely that these very young and emerging jets are still contained within their host galaxy, potentially providing favorable sites to observe the interactions between the young, compact jets and the intrinsic gaseous interstellar medium (ISM) near the central active galactic nuclei (AGN) within their galaxies (O'Dea \& Baum 1997, Owsianik \& Conway 1998, Readhead et al. 1996).

One of the best observational tracers of the intrinsic ISM is with observations of the cold neutral medium (CNM) as traced by the hyperfine spin-flip transition of neutral hydrogen, the HI $21 \mathrm{~cm}$ line. When cold neutral hydrogen $(\mathrm{T} \sim 100 \mathrm{~K})$ is present, absorption of the $21 \mathrm{~cm}$ line against these compact radio sources gives the ability to detect neutral hydrogen absorption systems at any redshift. The $21 \mathrm{~cm}$ absorption line could thus be an invaluable probe of the kinematics and morphology of the neutral gas in the nuclear regions of galaxies. Studies of HI quite often employ the use of compact radio

\footnotetext{
${ }^{1}$ Research School of Astronomy and Astrophysics, Australian National University, Canberra, ACT 2611, Australia; kathryn.grasha@anu.edu.au

2 ARC Centre of Excellence for All Sky Astrophysics in $3 \mathrm{Di}-$ mensions (ASTRO 3D), Australia

${ }^{3}$ Center for Astrophysics and Space Astronomy, Department of Astrophysical and Planetary Sciences, University of Colorado, 389 UCB, Boulder, CO 80309-0389, USA

${ }^{4}$ Department of Astronomy, University of Maryland, College Park, MD 20742-2421, USA

${ }^{5}$ Department of Astronomy, The Ohio State University McPherson Laboratory, 140 West 18th Avenue, Columbus, OH 43210-1173, USA
}

sources as the best targets to detect $21 \mathrm{~cm}$ absorbers as the jets are still contained within their host galaxies, providing insight to the possible feedback interactions of these young jets with the neutral ambient medium.

Since the seminal work of Roberts (1970) that identified extragalactic associated $\mathrm{HI} 21 \mathrm{~cm}$ absorption in NGC 5128, a large number of HI $21 \mathrm{~cm}$ absorption studies have been carried out in an attempt to provide a better understanding of the properties of neutral gas, especially within compact radio source host galaxies. The discovery of blueshifted HI absorption systems at large velocities $\left(\gtrsim-1000 \mathrm{~km} \mathrm{~s}^{-1}\right.$; Vermeulen et al. 2003) with respect to the systemic optical redshift of the galaxy hosting the compact radio source is indicative of jet-driven outflows of neutral gas. This signifies a unique avenue in which to study the fueling of the AGN as well as the effects of AGN feedback and its interaction with the environment and gas kinematics of the host galaxy.

The majority of HI $21 \mathrm{~cm}$ absorption systems have high column density and are damped Ly $\alpha\left(\mathrm{DLA} ; N_{\mathrm{HI}} \geq\right.$ $2 \times 10^{20} \mathrm{~cm}^{-2}$ ) systems. The vast majority of $21 \mathrm{~cm}$ absorption systems have low redshifts, $z \lesssim 1$ (for a compiled literature list, see Curran et al. 2016) but some of intrinsic absorbers do lie above $z \gtrsim 1$ (Uson et al. 1991, Moore et al. 1999; Ishwara-Chandra et al.||2003; Curran et al. 2013; Aditya et al. 2017; Aditya \& Kanekar 2018a). Previous surveys have identified a few commonalities exhibited in intrinsic HI $21 \mathrm{~cm}$ absorbers:

(1) High detection rates, of order $30-50 \%$, are found in surveys of compact radio sources at redshift $z \lesssim 1$ (e.g., Vermeulen et al. 2003; Pihlström et al. 2003; Gupta et al. 2006; Geréb et al. 2015; Maccagni et al. 2017) whereas the detection rates in higher redshift surveys are signif- 
icantly lower (e.g., Kanekar \& Chengalur 2003; Curran et al. 2008, 2011; Aditya et al. 2016, 2017; Curran et al. 2017 Moss et al. 2017). The deficiency of HI $21 \mathrm{~cm}$ absorbers at high redshitt could arise from detection biases in sample selection or could signify physical changes in the reservoirs of cold neutral gas, AGN activity, or radio emission at earlier cosmic time (although neutral gas fractions are generally expected to increase with redshift; Rhee et al. 2018, and references therein).

(2) No intrinsic $21 \mathrm{~cm}$ absorption system has ever been detected toward objects with an ultraviolet (UV) luminosity $L_{\mathrm{UV}}>10^{23} \mathrm{~W} \mathrm{~Hz}^{-1}$ (Curran et al. 2008, 2011, 2017) at any redshift. This luminosity is indicative of a UV bright, unobscured quasar with minimal extinction along the line of sight. While the exact nature of the UV luminosity's effect on neutral gas remains somewhat ambiguous, the observed threshold likely represents an analogue to the AGN "proximity effect" seen in UV absorption line studies (Bajtlik et al. 1988). The effect on the HI $21 \mathrm{~cm}$ absorption line could be the result of ionization of gas from the UV radiation (Curran et al. 2019), an increase in the spin temperature of the cold neutral medium (e.g., Kanekar \& Chengalur 2003), effectively decreasing the observed line optical depth for a given column density, or simply identifying systems that are unextincted and have little gas and dust along the line of sight.

(3) High redshift, optically bright AGN are most likely to have spectroscopic redshifts. Most radio sources with spectroscopic redshifts beyond $z \sim 1.2$ have UV luminosities in excess of $L_{\mathrm{UV}}>10^{23} \mathrm{~W} \mathrm{~Hz}^{-1}$. The UV luminosity threshold effect is therefore especially problematic for high redshift $21 \mathrm{~cm}$ absorption line detection because optical spectroscopic redshifts of AGN rely on rest-frame UV emission lines, biasing target selection toward the brightest UV sources that have never been detected in $21 \mathrm{~cm}$ absorption.

Another gas tracer that may be associated with neutral hydrogen absorption is the hydroxyl molecule $(\mathrm{OH})$. $\mathrm{OH}$ shows absorption, emission, and maser activity in four hyperfine and lambda-doubled transitions at $18 \mathrm{~cm}$. Cospatial $\mathrm{HI}$ and $\mathrm{OH}$ lines allow one to constrain the cosmic evolution of several physical constants: the electronproton mass ratio $\mu \equiv m_{p} / m_{e}$, the fine structure constant $\alpha=e^{2} / \hbar c$, and the proton g-factor $g_{p}$ (Chengalur \& Kanekar 2003; Darling 2003, 2004; Kanekar \& Chengalur 2004; Kanekar et al. 2005; Tzanavaris et al. 2005). The fine structure constant $\alpha$ parametrizes the strength of electromagnetic interactions, and some quasar studies suggest a decrease in $\alpha$ in the early universe (e.g., Cowie \& Songaila 1995, Murphy et al. 2001; Darling 2004, Tzanavaris et al.|2007; Kanekar et al. |2006; Srianand et al. 2010; Rahmani et al. 2012). Changes in the physical constants over cosmic time will cause spectral lines to shift from their relative measured values at present-day and have implications for physics and cosmology.

Redshifted $\mathrm{OH}$ systems are rarer than $\mathrm{HI} 21 \mathrm{~cm}$ absorption systems and are always found in systems that are detected in $21 \mathrm{~cm}$ absorption as well. Five $\mathrm{OH} 18 \mathrm{~cm}$ absorption line systems are known to date at $z>0.2$ Chengalur et al. 1999, Kanekar et al. 2003, Darling 2004, Kanekar et al. 2005, 2012), and none lie beyond $z=1$. Compact radio sources still confined within the host galaxy are the perfect environment to search for molecular systems, producing optimal conditions to observed $\mathrm{OH}$ absorption lines, especially the conjugate ${ }^{6} \mathrm{OH}$ lines, systems in which $\mathrm{OH}$ gas complexes become sources of coupled weak maser emission and non-thermal absorption. Large surveys over a broad redshift range are required to detect the rare coincidence of $\mathrm{HI} 21 \mathrm{~cm}$ and $\mathrm{OH} 18 \mathrm{~cm}$ absorption lines.

In this paper, we perform a large survey for intrinsic HI $21 \mathrm{~cm}$ and $\mathrm{OH} 18 \mathrm{~cm}$ absorption in the host galaxies of 145 compact radio sources, including GHz-Peaked Spectrum (GPS), Compact Steep-Spectrum (CSS), Compact Symmetric Objects (CSOs), and Flat-Spectrum Radio Sources (FSRSs; see Section 2 for further discussion of these classifications), using the Green Bank Telescope ${ }^{7}$ (GBT) selected across the redshift range $(0.02<z<3.8)$ in an attempt to increase the number of known absorbers, especially at high redshift. In addition to the results of our search for intrinsic $\mathrm{HI}$ and $\mathrm{OH}$ absorption, we examine several biases that influence the detection of intrinsic absorption, including redshift selection, UV luminosity, radio luminosity, radio source size, covering factor, and spin temperature. We explore the possibility that the nature of our high redshift selection of radio sources biases the results against detection of intrinsic $21 \mathrm{~cm}$ absorption. Previous $21 \mathrm{~cm}$ absorption studies of the same biases we investigate here were performed on a heterogeneous compilation of previous known intrinsic HI absorption with a wide range of source sizes that were typically located at low redshift (e.g., Vermeulen et al. 2003: Gupta et al. 2006; Geréb et al. 2014). 'These results are arguably not unbiased because non-detections have generally not been uniformly published, which will skew the detection rates. We use this large survey to test the UV luminosity threshold hypothesis and the high rate of intrinsic HI $21 \mathrm{~cm}$ absorption toward compact radio sources.

The paper is outlined as follows. The compact radio source sample selection is described in Section 2 and their observations are described in Section 3 . The results and comparison to previous $21 \mathrm{~cm}$ absorption searches are presented in Section 4. Section 5 presents the analysis of the HI detection rate with the radio and UV luminosities. We discuss the findings in Section 6 and we summarize the results and conclusions in Section 7 .

Throughout this paper, we adopt a flat $\Lambda \mathrm{CDM}$ cosmology with $\Omega_{\mathrm{m}}=0.27, \Omega_{\Lambda}=0.73$, and $H_{\circ}=$ $71 \mathrm{~km} \mathrm{~s}^{-1} \mathrm{Mpc}^{-1}$ (Komatsu et al. 2011). All quantities obtained from the literature are recalculated (if necessary) for this cosmology.

\section{SAMPLE SELECTION}

Compact radio sources are often classified by their morphology and radio spectral index, providing insight

6 The satellite $\mathrm{OH}$ conjugate lines (1612 and $1720 \mathrm{MHz}$ ) are a rare astrophysical phenomenon where the two lines have the same shape but opposite signs, arising due a population antiinversion mechanism resulting from quantum mechanical selection rules (Elitzur 1992, van Langevelde et al. 1995 Darling 2004 Kanekar et al. 2018).

The National Radio Astronomy Observatory is a facility of the National Science Foundation operated under cooperative agreement by Associated Universities, Inc. 
into the properties and nature of these sources. GHzPeaked Spectrum (GPS) sources are intrinsically small (i.e., not foreshortened by projection effects; Fanti et al. 1990 and show a low frequency turn-over in their spectra, mainly attributed to synchrotron self-absorption and free-free (thermal bremsstrahlung) absorption (Jones et al. 1974, Menon 1983, O’Dea \& Baum 1997). Compact Steep-Spectrum (CSS) sources show steeper powerlaw spectra than is typically seen in radio galaxies (Peacock \& Wall 1982). Compact Symmetric Objects (CSOs) show a compact double-lobed structure with advance speeds, indicative of young jets (Wilkinson et al. 1994 Owsianik \& Conway 1998). Flat-Spectrum Radio Sources (FSRSs) are characterized with a double-peaked synchrotron/Compton spectral energy distribution, possibly associated with a blazar or with the compact core of a radio galaxy (Fugmann 1988, Molina et al. 2012). These classifications are not disjoint in the literature; for example, most CSOs are also classified as GPS sources (Xiang et al. 2005).

Candidate intrinsic HI $21 \mathrm{~cm}$ absorbers in this survey include nearly all known GPS sources, CSS sources, and CSOs (ca. 2005) with $\delta>-35^{\circ}$ (Spencer et al. 1989, Fanti et al. 1990, de Vries, Barthel, \& O'Dea 1997; Morganti et al. 1997; O'Dea \& Baum 1997; Peck \& Taylor 2000 Xiang et al. 2005) for a total of 60 objects (34 GPS sources, 29 CSS sources, and 7 CSOs, with some overlap between the classifications). The FSRSs were selected from White \& Becker (1992) with $S>0.3$ Jy at $780 \mathrm{MHz}$, adding an additional 85 sources to the sample selection.

In total, the sample includes 145 objects with known optical redshifts $0<z<4$, with the vast majority of the sample at redshifts less than $z \sim 1$. Figure 1 shows the redshift and the continuum flux density at the expected redshifted line frequency of the two samples (compact and flat-spectrum sources; Section 4.2). Table 1 lists the sources, their radio classifications (GPS, CSS, CSO, and FSRS), total integration time for each reduced spectrum.

\section{OBSERVATIONS AND DATA REDUCTION}

From September 2004 to August 2005, we observed 85 FSRSs for intrinsic absorption in the HI $21 \mathrm{~cm}$ line $(1420.405752 \mathrm{MHz})$, and 81 in the four $18 \mathrm{~cm} \mathrm{OH}$ lines at $1612.231,1665.4018,1667.35903$, and $1720.5300 \mathrm{MHz}$ simultaneously (GBT program 04C-018). We additionally observed 60 GPS, CSS, and CSO radio sources for intrinsic absorption from December 2005 to May 2006 (GBT program 06A-044): 59 sources were observed in the $\mathrm{HI}$ $21 \mathrm{~cm}$ line, and 44 sources were observed in the four $\mathrm{OH}$ $18 \mathrm{~cm}$ lines. One source, PKS 2135-209, was only observed in the $\mathrm{OH}$ lines. All observations were conducted using a 5-minute position-switched mode in two linear polarizations with 9-level sampling, 3- to 15-sec records, and a $50 \mathrm{MHz}$ bandwidth centered at the redshift of the radio source host galaxy. Each bandwidth contains 4096 spectral channels with a width of $12.2 \mathrm{kHz}$ per channel (a channel width of $3.86 \mathrm{~km} \mathrm{~s}^{-1}$ at $z=0.5$ ). A calibration diode signal was injected for half of each record. Total on-source integration times were typically 30 minutes for the GPS, CSS, and CSO sources and 5 minutes for the FSRSs, listed in Table 1

Each off-source-flattened and diode-calibrated spectral record was inspected and flagged for radio frequency interference (RFI). All records were then averaged in time and polarization, Hanning smoothed, and baselineflattened using a polynomial fit, typically of third order with a final post-smoothing spectral resolution of $24.4 \mathrm{kHz}$ per channel. All data reduction was performed using GBTIDL $^{8}$. Final mean spectra were inspected for absorption lines within a few thousand $\mathrm{km} \mathrm{s}^{-1}$ of the optical redshift of the host galaxy. The observable velocity span was typically determined by the RFI conditions rather than the spectral bandwidth.

\section{RESULTS}

The nature of 36 sources (25\% of the total sample) remains ambiguous due to unrecoverable contamination of RFI, a pernicious impediment to the detection of new absorption systems. The remaining 108 sources have the majority of their spectral range free from RFI and are able to be searched for $\mathrm{HI}$ or $\mathrm{OH}$ absorption.

Table 2 lists the RFI-free velocity range searched for HI $21 \mathrm{~cm}$ absorption toward each radio source, the measured rms noise (away from RFI and spectral lines) in the mean spectra, the continuum flux density at the expected frequency of the redshifted spectral line obtained from power-law fits to extant continuum measurements in the literature (see Section 4.2), HI column densities (or upper limits toward radio sources not detected in $21 \mathrm{~cm}$ absorption), and the projected size of each radio source as obtained from literature searches (see Section 5.3).

Table 3 lists the properties of the $\mathrm{OH} 18 \mathrm{~cm}$ line observations toward a subset of the sample. The 1665 and $1667 \mathrm{MHz}$ lines are both observed, but we only list the properties of the $1667 \mathrm{MHz}$ line because it places the strongest upper limit on the $\mathrm{OH}$ column density among the $18 \mathrm{~cm}$ lines. The continuum flux density is interpolated at the expected frequency of the redshifted $1667 \mathrm{MHz}$ line from continuum measurements in the literature following the same method used for the $21 \mathrm{~cm}$ spectral observations (Section 4.2). When the $1667 \mathrm{MHz}$ line could not be observed due to RFI, the 1612 or $1720 \mathrm{MHz}$ transitions are used as the reference instead.

\subsection{Line Detections and Limits}

We detect intrinsic HI $21 \mathrm{~cm}$ absorption in 6 of the 108 RFI-free compact radio sources in our sample. The detection rate is $5.6 \%$, substantially lower than in previous intrinsic HI absorption line surveys (Section 6). All of these absorption lines are detected in previous surveys (see Table 2 for references). The detected line spectra are shown in Figure 2, where velocities are referenced to the optical heliocentric redshift. Table 4 lists the measured and derived properties of the detected HI $21 \mathrm{~cm}$ absorption lines.

Table 2 lists the measured upper limits to the column densities, calculated according to Section 4.3.1 and further discussed in light of previous studies in Section 4.4.1. We show the spectra of the 102 RFI-free sources that show no significant HI $21 \mathrm{~cm}$ absorption lines and are predominately free from RFI in the Appendix.

8 GBTIDL (http://gbtidl.nrao.edu/) is the data reduction package produced by NRAU and written in the IDL language for the reduction of GBT data. 
PKS $0742+10$, at $z=2.64$, shows a redshifted negative spectral feature with a signal to noise of $3.4 \sigma$. We compare our spectrum to that of Curran et al. (2013), also obtained at the GBT. The Curran et al. (2013) spectrum shows narrow RFI at the frequency of the potential absorption line. This suggests that the feature in our spectrum is likely to be an incompletely flagged or low-level RFI feature. We therefore treat PKS $0742+10$ as a nondetection in all subsequent figures and statistics, with the rms noise measured away from the spectral feature.

None of the four $18 \mathrm{~cm} \mathrm{OH}$ lines are detected in absorption or emission in 102 RFI-free spectra out of the 125 objects observed. Table 3 lists the properties of the $\mathrm{OH}$ sample, and Figure 15 in the Appendix shows the $\mathrm{OH} 1667 \mathrm{MHz}$ non-detection spectra of the four $21 \mathrm{~cm}$ absorbers in our sample with RFI-free $\mathrm{OH}$ spectral observations.

\subsection{Flux Densities}

The nature of our observations with the GBT maximizes the on-time observation of targets and as a result does not give the necessary observations to flux calibrate the data and directly measure the continuum flux density. Moreover, for position-switched observations, RFI and instrumental effects frequently result in negative continuum values, artificial continuum slopes, and bandpass structure. The continuum is however paramount to obtain the optical depth and measure the column density or place a limit in the cases of non-detections (see eqn. 1). We therefore obtain the continuum flux density at the frequency of the redshifted $\mathrm{HI}$ and $\mathrm{OH}$ line of each source from an interpolation between extant continuum measurements in the NASA Extragalactic Database (NED) using a single spectral index power-law fit (a linear fit in $\log S_{\nu}-\log \nu$ space). In order to cope with the heterogeneous literature continuum measurements, we try whenever possible to select values published from the same catalogs and pass bands, most often observations at $1410,1340,750,635,408$, and $365 \mathrm{MHz}$ (Laing \& Peacock 1980; Large et al. 1981; Ficarra, Grueff, \& Tomassetti 1985, White \& Becker 1992; Douglas et al. 1996; Rengelink et al.|1997; Stanghellini et al.|1998, Condon et al. |1998; Stanghellini et al. |2005; Orienti et al. |2007; Petrov et al. 2008). The uncertainties in the interpolated continuum Hux densities listed in Tables 2 and 3 are obtained from estimates of the $1 \sigma$ confidence intervals of the power law continuum fits for each object. This treatment neglects potentially significant time variation in radio source fluxes.

\subsection{Column Densities}

\subsubsection{HI Column Density}

Column densities are derived from the integrated optical depths of spectral lines. The column density associated with a given 21-cm line optical depth is calculated according to Wolfe \& Burbidge (1975) as

$$
N_{\mathrm{HI}}=\left(1.8 \times 10^{18} \mathrm{~cm}^{2}\right) \frac{T_{s}}{f} \int \tau d v,
$$

where $T_{s}$ is the spin temperature of the $21 \mathrm{~cm}$ line in Kelvin, $f$ is the fraction of the area of the continuum source covered by the absorber, and $\int \tau d v$ is the optical depth $\tau$ integrated across the velocity span of the line, in $\mathrm{km} \mathrm{s}^{-1}$, calculated from the continuum fluxes acquired in Section 4.2 and the strength of the absorption line. A Gaussian absorption profile can be integrated: $\int \tau d v=\sqrt{\pi / \ln 2} \tau_{\max } \Delta v / 2$, where $\tau_{\max }$ is the peak optical depth of a line with a full width half $\max$ (FWHM) of $\Delta v$. The column densities of the six re-detections of 21 $\mathrm{cm}$ absorption are calculated according to Eq. 11 and are listed in Table 4. We use both Gaussian fits and direct integration for the total HI column density of the $21 \mathrm{~cm}$ detections.

For the non-detections, we assume optically thin lines, and the $3 \sigma$ upper limit on the column density can be approximated by

$$
N_{\mathrm{HI}, 3 \sigma}<\left(1.9 \times 10^{18} \mathrm{~cm}^{-2}\right) \frac{T_{s}}{f} \tau_{3 \sigma} \Delta v,
$$

where $\tau_{3 \sigma} \approx 3 \sigma / S, \sigma$ is the rms noise, and $S$ is the continuum flux density of the radio source at the redshifted line frequency.

All HI column densities are computed assuming $T_{s}=$ $100 \mathrm{~K}$ and a uniform covering factor of $f=1$, resulting in an HI column density limit in the CNM phase of the ISM. We note that this is not the total HI column density as the $21 \mathrm{~cm}$ transition is not a reliable tracer of the gas in WNM due to the optical depth inversely related to the spin temperature.

Non-detection column density limits assume $\Delta v=$ $30 \mathrm{~km} \mathrm{~s}^{-1}$. Table 2 lists the column densities and limits for the non-detections and all upper limit spectra are shown in the Appendix. Figure 3 shows the HI column density distribution as a function of redshift for the 108 RFI-free sources.

\subsubsection{OH Column Density}

For the redshifted $\mathrm{OH} 18 \mathrm{~cm}$ line non-detections, we use the $1667 \mathrm{MHz} \mathrm{OH}$ transition to place the strongest upper limit on the $\mathrm{OH}$ column densities via

$$
N_{\mathrm{OH}, 3 \sigma}<X \times 1.0645 \frac{T_{x}}{f} \tau_{3 \sigma} \Delta v,
$$

where $X=2.38 \times 10^{14} \mathrm{~cm}^{-2}$ Curran et al. 2008) for the $1667 \mathrm{MHz}$ transition, and $T_{x}$ is the excitation temperature of the $\mathrm{OH} 1667 \mathrm{MHz}$ line. When the $1667 \mathrm{MHz}$ line could not be observed due to RFI, the $\mathrm{OH}$ column density limit is calculated using the $1612 \mathrm{MHz}$ or $1720 \mathrm{MHz}$ transition, which have $X=2.14 \times 10^{15} \mathrm{~cm}^{-2}$ $\left(X_{1612 \mathrm{MHz}}=9 \times X_{1667 \mathrm{MHz}}\right.$, following the $18 \mathrm{~cm} \mathrm{OH}$ line ratios in thermodynamic equilibrium). We make no assumption about the $\mathrm{OH}$ line excitation temperatures and leave $T_{x}$ as a free parameter in our tabulation and analysis. As with the $\mathrm{HI}$ lines, for the $\mathrm{OH}$ lines we assume a uniform covering factor of $f=1$, and a line width of $\Delta v=30 \mathrm{~km} \mathrm{~s}^{-1}$. Figure 4 shows the $\mathrm{OH}$ column density limits as a function of redshift for the 102 RFI-free sources in the sample.

Because we find no $\mathrm{OH}$ detections, as well as no new $\mathrm{HI}$ detections, we are unable to place any meaningful constraint on possible redshift variations in the fundamental physical constants, discussed in Section 1

\subsection{Comparison to Previous Work} 4.4.1. HI $21 \mathrm{~cm}$ Absorption Studies 
This intrinsic HI $21 \mathrm{~cm}$ absorption search toward 144 compact radio sources (Table 1 one additional source is only observed for the four $\mathrm{OH}$ lines, for a total sample size of 145) obtained 108 spectra including six redetections, with 36 sources remaining ambiguous due to RFI. 21 objects in the survey sample are previously observed for intrinsic HI absorption including nine known $21 \mathrm{~cm}$ line absorbers in the sample.

We made no new $21 \mathrm{~cm}$ absorption detections, however, we do re-detect six of the nine known HI absorption systems (Table 4). We are not able to re-detect the three absorption line systems toward 3C49, B3 1355+441 (Vermeulen et al. 2003) or 3C190 (Ishwara-Chandra et al. $2003)$ due to RFI.

Our spectra of 3C138, 3C147, 0239+108, OE+131, TXS $0902+490, \quad$ HB89 $0954+658, \quad 1004+141$, FBQS J1159+2914, 1418+546, 1642+690, 3C380, $4 \mathrm{C}+29.56,2007+222$, and PKS 2149+056 improves the previous upper limit measurements (Vermeulen et al. 2003, Carilli et al. 1998; Curran et al. 2017, 2019; Aditya \& Kanekar 2018a b). Three objects, B3 0248+430, SBS $0804+499$, and 3C147, were impacted by RFI in our observations, preventing independent upper limits on previous measurements. Five sources, 3C138, HB89 0754+100, COINSJ 1546+0026, PKS 2121-01, and HB89 2342+821 have column density limits comparable to previous surveys. Only two limits on our sources, B2 0738+31 and COINS J2022+6136, did not improve on previously published column density limits. The remaining 79 sources have (to our knowledge) never been searched for intrinsic HI absorption before. Table 2 lists the column densities or column density limits from our survey and previous surveys along with references, if applicable. All non-detections quoted at the $3 \sigma$ level use the assumptions listed in Section 4.3 .

The six detected absorbers all have column densities near or greater than the DLA limit of $\mathrm{N}_{\mathrm{HI}}>$ $2 \times 10^{20} \mathrm{~cm}^{-2}$ and we reached adequate sensitivity to have detected most known redshifted $21 \mathrm{~cm}$ absorbers if they had been present in our sample. $70 \%$ of our nondetections have $3 \sigma$ upper limits below the DLA column density threshold for the detected absorbers. This suggests that while we have the sensitivity to detect new systems of cold gas (T 100 K) at sub-DLA column densities, there must be physical reasons for the dearth of absorption lines in these redshifts radio sources in this survey, which we investigate further in Section 5.2 .

\subsubsection{OH $18 \mathrm{~cm}$ Absorption Studies}

We searched 125 radio sources for the four $\mathrm{OH} 18 \mathrm{~cm}$ lines, which can appear in either emission or absorption. The spectra of 102 objects are unaffected by RFI for at least one of the four $\mathrm{OH}$ transitions. No $\mathrm{OH}$ emission or absorption lines are detected in any of the RFI-free spectra and the $\mathrm{OH}$ column density upper limits are reported in Table 3. Only one source in our sample, B3 1355+441, has prior $\mathrm{OH}$ observations (Curran et al. 2006). We report a $3 \sigma$ upper limit on the $\mathrm{OH}$ column density of $N_{\mathrm{OH}} / T_{x}<2.1 \times 10^{13} \mathrm{~cm}^{-2} \mathrm{~K}^{-1}$, an improvement over of the $3 \sigma$ measurement of $N_{\mathrm{OH}} / T_{x}<11 \times 10^{13} \mathrm{~cm}^{-2} \mathrm{~K}^{-1}$ from Curran et al. (2006).

Four RFI-free $\mathrm{OH}$ observations toward known $21 \mathrm{~cm}$ absorbers (Figure 15 do not detect any $\mathrm{OH}$ lines despite reaching very low optical depths, $0.002<\tau_{3 \sigma}<0.01$
(Table 3). OH column density upper limits for the full sample span the range $10^{12} \mathrm{~cm}^{-2} \mathrm{~K}^{-1}<N_{\mathrm{OH}} / T_{x}<$ $10^{16} \mathrm{~cm}^{-2} \mathrm{~K}^{-1}$, consistent with upper limits quoted in previous surveys for non-detections of $\mathrm{OH} 18 \mathrm{~cm}$ absorption (Gupta et al. 2006, Curran et al. 2008, 2011).

\section{ANALYSIS}

We detect no new $21 \mathrm{~cm}$ absorption systems in our survey. We do, however, re-detect six known absorbers, for a detection rate of $5.6 \%$. The following sections discuss factors that may negatively impact the ability to detect neutral HI gas, especially at high-redshift.

\subsection{Radio Continuum Luminosity}

One possible explanation of our low detection rate, especially at high redshift, is a high spin temperature. As shown in Equation 2, the measured HI column density is proportional to the (unknown) spin temperature $T_{s}$ of the absorbing gas. Previous studies investigated the impact of the local radio radiation field in driving the spin temperature up in associated absorption systems (e.g., Gupta et al. 2006: Curran \& Whiting 2010), finding little to no significance dependence of the radio luminosity on the detection rate or $N_{\mathrm{HI}}$. If the spin temperature is coupled to the local radio radiation temperature, an increase would cause weaker line absorption for a given column density. However, recent work by Aditya \& Kanekar $(2018 \mathrm{a}$ b) suggest that gas excitation from the radio continuum may be responsible for the dearth of 21-cm absorbers detected at high redshift. This suggests that the spin temperature of the gas, a measure of the excitation from the lower hyperfine level (Field 1959), can be raised by excitation to the upper hyperfine level by rest-frame $1420 \mathrm{MHz}$ photons. Curran et al. (2019) has shown that this is unlikely and that the photoionization from the UV luminosity is dominant factor over the radio luminosity in negatively impacting the detection rate of $21 \mathrm{~cm}$ absorbers (see Section 5.2).

We test this possibility of the impact of the incident $21 \mathrm{~cm}$ luminosity on the absorption detection rate by calculating the specific radio continuum luminosity in the vicinity of the $21 \mathrm{~cm}$ line in the rest frame of each host galaxy (van Gorkom et al. 1989):

$$
L_{\nu}=\frac{4 \pi D_{\mathrm{L}}^{2} S_{\nu}}{1+z} \mathrm{~W} \mathrm{~Hz}^{-1},
$$

where $D_{\mathrm{L}}$ is the luminosity distance of the host galaxy, $S_{\nu}$ is the observed $21 \mathrm{~cm}$ flux density at the frequency of the redshifted $21 \mathrm{~cm}$ observations, as calculated in Section 4 and listed in Table 2 , and $z$ is the redshift.

Table 5 lists the radio continuum luminosities for the sample. The median radio continuum luminosity of the detections is $\log \left(L_{\text {Radio }} / \mathrm{W} \mathrm{Hz}^{-1}\right)=27.5 \pm 0.4$, consistent with the median luminosity for the non-detections at $27.5 \pm 0.7$. A Kolmogorov-Smirnov Test (KS-test) shows that the $21 \mathrm{~cm}$ rest-frame luminosity differs between the detections and non-detections at less than $1 \sigma$ confidence level; the $21 \mathrm{~cm}$ luminosity does not appear to be a major factor in $21 \mathrm{~cm}$ absorption and cannot explain our low detection rate, also found in Curran \& Whiting (2010).

With the exception of a few low-luminosity radio sources, the radio luminosity-redshift distribution shows 
the Malmquist bias for flux-limited surveys: the most luminous objects lie at the largest distances (Figure 5). As expected, the detections show an inverse correlation of radio luminosity with column density (Figure 6). This, however, is an observational bias because the highest flux density sources typically have the highest luminosities (Figure 5), and high flux densities provide greater column density sensitivity.

\subsection{Ultraviolet Luminosity}

Another possible explanation for this survey's lack of intrinsic $21 \mathrm{~cm}$ absorption at high redshift is the hypothesis proposed by Curran et al. (2008) that AGN with a high ultraviolet $\left(\lambda_{\mathrm{UV}}=1216 \AA\right.$ A $)$ luminosity $\left(L_{\mathrm{UV}}>10^{23} \mathrm{~W} \mathrm{~Hz}^{-1}\right)$ may inhibit $21 \mathrm{~cm}$ absorption. If the UV flux of the central AGN is sufficient to ionize (or simply heat) the neutral gas within the host galaxy, UV-bright galaxies will be biased against the detection of $21 \mathrm{~cm}$ absorbers. These UV bright sources are also likely to be unextincted and may simply have less gas and dust along the line of sight compared to extincted sources, lowering the ability to detect absorption systems as sensitivity depends on both the covering fraction of the gas against the background source and the spin temperature of the gas. The UV luminosity bias will be compounded with increasing redshift because flux-limited surveys will naturally select UV-luminous objects at all redshifts.

We have searched the literature for optical and near-IR photometry of each source in our sample to estimate the rest-frame UV luminosity following the method in Curran et al. (2008). Table 5 lists the optical and near-IR magnitudes for the sources searched for $21 \mathrm{~cm}$ absorption. Of our detected absorbers, $4 \mathrm{C}+76.03$ does not have adequate available optical or near-IR observations. For this source, along with six sources with non-detections, we are unable to estimate the UV-optical slope in order to measure the expected UV luminosity. We therefore do not calculate a UV luminosity for these sources and they have been excluded from all plots and analysis related to the UV luminosity. In conclusion, we have five detections in $21 \mathrm{~cm}$ absorption and 96 sources with upper limits that we are able to calculate a UV luminosity for in order to test the hypothesis proposed by Curran et al. (2008) for the ionization of the atomic gas in UV-bright radio sources.

Using visible band magnitudes and attempting to maintain consistency in the heterogeneous sample and sources of magnitudes, the extrapolation or interpolation of the rest-frame UV luminosity at $1216 \AA$ is performed using a power-law fit to the $B$ and $R$ bands as these two bands are readily available for most for the sample. For sources where either of those bands are not available, observations at $V$ or $K$ bands are substituted in that order. We require a minimum of two bands to perform the power-law fit, for galaxies with more than two available magnitudes, we use all available optical and near-IR bands. Objects with Sloan Digital Sky Survey (SDSS) $u^{\prime} g^{\prime} r^{\prime} i^{\prime} z^{\prime}$ magnitudes are converted to Johnson magnitudes using the methods described in Smith et al. (2002) and are not corrected for intrinsic source extinction.

The fitting is done by converting the visible band magnitudes to fluxes using Vega as a zero-point reference, with values for Vega in each band taken from
Bessell (1979), Campins et al. (1985), and Schneider et al. (1983). Under the assumption that a single power law applies from the observed optical wavelengths down to $1216 \AA$, the UV flux density in the rest-frame of the host galaxy is obtained from the observed flux $F_{1}$ at wavelength $\lambda_{1}$ from Curran et al. (2008) as,

$$
F_{\lambda_{\mathrm{UV}}(1+z)}=F_{1}\left[\frac{\lambda_{\mathrm{UV}}(1+z)}{\lambda_{1}}\right]^{\alpha},
$$

where the spectral index $\alpha$ is estimated as $\alpha=$ $\log \left(F_{2} / F_{1}\right) / \log \left(\lambda_{2} / \lambda_{1}\right)$ for two observations at bands $\lambda_{1}$ and $\lambda_{2}$, with fluxes $F_{1}$ and $F_{2}$. This allows us to extrapolate from the observed visible-band photometry to obtain a UV flux at the observed wavelength $\lambda_{\mathrm{UV}}(1+z)$. The specific UV luminosity in the rest-frame of the host galaxy is then calculated as,

$$
L_{\lambda_{\mathrm{UV}}}=\frac{4 \pi D_{\mathrm{L}}^{2} F_{\lambda_{\mathrm{UV}}(1+z)}}{1+z} \mathrm{WHz}^{-1}
$$

where $D_{\mathrm{L}}$ is the luminosity distance to the host galaxy and $F_{\lambda_{\mathrm{UV}}(1+z)}$ is the rest-frame UV flux calculated from Eq. 5 .

The median and $1 \sigma$ values of the UV luminosity of the full sample of 101 sources (5 detections, 96 non-detections, 7 sources with no $\mathrm{L}_{\mathrm{UV}}$ measurement) is $\log \left(L_{\mathrm{UV}} / \mathrm{W} \mathrm{Hz}^{-1}\right)=23.1 \pm 1.4$, consistent with the UV luminosity threshold of $10^{23} \mathrm{~W} \mathrm{~Hz}^{-1}$. The median luminosity of the $\mathrm{HI}$ absorption detections is $\log \left(L_{\mathrm{UV}} / \mathrm{W} \mathrm{Hz}^{-1}\right)=20.5 \pm 1.1$, and the median luminosity of the non-detections is $\log \left(L_{\mathrm{UV}} / \mathrm{W} \mathrm{Hz}^{-1}\right)=$ $23.2 \pm 1.4$. If we only consider systems below the $L_{\mathrm{UV}}<$ $10^{23} \mathrm{~W} \mathrm{~Hz}^{-1}$ threshold, this reduces the number of RFIfree sources searched for $21 \mathrm{~cm}$ absorption from 101 to 47 targets. The detection rate for this subset of UV-faint sources increases to $9.8 \%$.

Figure 7 shows the distribution of the HI column density as a function UV luminosity. There are no HI $21 \mathrm{~cm}$ line absorbers associated with sources with UV luminosities above $\log \left(L_{\mathrm{UV}} / \mathrm{W} \mathrm{Hz}^{-1}\right)>23$, further supporting the critical UV threshold above which $21 \mathrm{~cm}$ absorbers cannot be detected (Curran \& Whiting 2010, Curran et al. 2017). Figure 8 shows the UV luminosity as a function of redshift, demonstrating how the radio sources in this survey above a redshift of $z \sim 1$ predominately have UV luminosities that are above the threshold. The requirement of UV/optical spectroscopic redshifts for radio spectral line surveys typically cause the high redshift sources to be the most luminous and are not ideal targets to show $21 \mathrm{~cm}$ or molecular absorption.

A KS-test comparing UV luminosities of the $21 \mathrm{~cm}$ detections to the non-detections, however, is not statistically significant, showing a $2.2 \sigma$ difference between the samples. While the detections and non-detections do not appear to be drawn from distinct populations in UV luminosity, our sample of detections may simply be too small for statistical significance.

Figure 9 shows the radio versus UV luminosities of the sample with a Pearson correlation coefficient of $r=0.35$, significant at the $3.7 \sigma$ level. As with all luminosityluminosity plots of flux-limited samples, some degree of correlation can arise from the correlation of both luminosities with distance. Figure 10 shows the measured 
column density (or $3 \sigma$ upper limits) versus the ratio of the radio-to-UV luminosity. The five HI $21 \mathrm{~cm}$ absorbers have a weak preference be located in objects that have a higher than median ratio, indicating their preference to reside in sources with low UV luminosity and/or higher radio luminosity compared to the non-detected sources. A KS test results in a D-value of 0.677 , significant at $3.2 \sigma$. Future work with a larger number of detections will be able to further investigate the effect of the UV and radio luminosities on cold gas, especially investigating preference for $21 \mathrm{~cm}$ absorption in UV-faint, radio-loud sources.

\subsection{Physical Size}

The size of the radio source may also influence the detection of $\mathrm{HI} 21 \mathrm{~cm}$ absorption. If the radio lobes predominately reside outside of the host galaxy, then the HI covering fraction will be small, and the apparent $21 \mathrm{~cm}$ optical depth will be correspondingly diminished.

The sizes of the radio sources in our survey are compiled from existing literature (Table 2). Physical and projected sizes are calculated using the angular size distance for each redshift. Projection (and presumably relativistic beaming) can confuse analysis and interpretation because not all sources with small projected linear sizes are physically small and vice versa (see, e.g., Fanti et al. 1989).

Figure 11 shows the HI column densities (upper limits in the case of non-detections) as a function of the projected linear size of the radio source. The detection of absorption systems does not seem to depend on source size and we find no correlation between the column density and the size of the source (Pearson correlation coefficient of -0.31$)$. The detection rates among the larger sources ( $>1 \mathrm{kpc} ; 2$ out of 21 sources) and the smaller sources ( $<1 \mathrm{kpc} ; 4$ out of 87 sources) are consistent with each other.

Figure 12 shows that UV luminosity does not correlate with source size (correlation coefficient of $r=$ 0.14 , non-significant at $1.4 \sigma)$. Among the detections, the larger sources do exhibit a higher UV luminosity $\left(L_{\mathrm{UV}}>10^{22.5} \mathrm{~W} \mathrm{~Hz}^{-1}\right)$ than the smaller sources $\left(L_{\mathrm{UV}}<10^{21} \mathrm{~W} \mathrm{~Hz}^{-1}\right)$, but the correlation is not statistically significant for a sample of five detections. Among non-detections, smaller sources $(\leq 1 \mathrm{kpc})$ show a median UV luminosity of $\log L_{\mathrm{UV}}=23.0 \pm 1.3 \mathrm{~W} \mathrm{~Hz}^{-1}$, consistent with that of the larger sources $(>1 \mathrm{kpc})$ with a median of $\log L_{\mathrm{UV}}=22.6 \pm 1.5 \mathrm{~W} \mathrm{~Hz}^{-1}$. The median UV luminosities likewise do not differ among radio source classification types: the median UV luminosity of the GPS, CSS, and CSO sources is $\log L_{\mathrm{UV}}=$ $22.8 \pm 1.6 \mathrm{~W} \mathrm{~Hz}^{-1}$, while for FSRSs, the median value is $\log L_{\mathrm{UV}}=23.1 \pm 1.2 \mathrm{~W} \mathrm{~Hz}^{-1}$.

Figure 13 shows the relation between the radio continuum flux density and linear size of the radio jets, where the smallest radio sources tend to also be the faintest. These properties are countervailing in terms of the HI $21 \mathrm{~cm}$ absorption line detectability. Column density sensitivity increases toward brighter objects, which tend to occur in the largest radio sources. However, the largest radio sources are the most evolved (thus possibly residing in galaxies with less neutral gas) and most likely have the smallest covering fraction if the radio lobes are located outside the galaxy, reducing sensitivity for systems with lower column densities. We do not find that smaller sources have a higher rate of detection in $21 \mathrm{~cm}$ absorption, instead, we find that the detected absorbers cover the full range of jet sizes available in the survey, concluding that the size of the radio source is not a main contributor against the detection of $\mathrm{HI} 21 \mathrm{~cm}$ absorption.

\section{DISCUSSION}

Some of the first surveys toward compact radio sources revealed intrinsic HI $21 \mathrm{~cm}$ detection rates of 30-60\% in low redshift radio galaxies (Morganti et al. 2001; Pihlström et al. 2003; Vermeulen et al. 2003; Gupta et al. 2006). These previous surveys measure typical optical depth limits of $\tau_{3 \sigma} \lesssim 0.001-0.2$ for non-detections. We recover a mean optical depth limit of $\tau_{3 \sigma}<0.023$ for our non-detections with a broad range from $10^{-6}<\tau_{3 \sigma}<$ 0.15 , where $70 \%$ of the non-detections lie below the DLA column density threshold. For sources in our survey that were also present in previous studies, we typically achieve greater sensitivity and are able to improve prior upper limits.

The high success rate of $21 \mathrm{~cm}$ absorbers in these low-z studies have triggered follow up surveys to target compact radio sources in attempt to increase the detection rate of $21 \mathrm{~cm}$ absorption systems. The high success rates of these low-redshift surveys are in stark contrast, however, with surveys aimed toward high redshift $z \gtrsim 1$ targets, where the detection rate plummets to single digits (Curran et al. 2008, Aditya et al. 2016; Curran et al. 2017), hinting at a connection between the redshift of the source and the success of detecting $21 \mathrm{~cm}$ absorption lines. While our sources span the redshift range $0.2<z<3.8,75 \%$ of our RFI-free sample lie at redshifts $z<1$ and we recover a detection rate of less than $6 \%$, with all absorbers identified in prior surveys. Lack of new detections, especially at low redshift, while reaching the sensitivity to re-detect known absorbers, demonstrates that there have to be external physical factors at play that negatively impact the detection rate of neutral gas absorbers in our sample.

It is becoming clear that absorption of cold, neutral gas within galaxies that host radio sources is a multifaceted problem, with many factors impacting the detection rate of $\mathrm{HI} 21 \mathrm{~cm}$ absorption, especially at high redshift. Different effects may influence each other and work together, such as the observed anti-correlation of the HI column densities with size of the radio source (Curran et al. 2013: Geréb et al. 2015). We do not recover the same correlation, although our data are not inconsistent with a decrease in the HI column density with increase in source size (Figure 11). Our results are limited by an extremely small number of detections. Surveys such as this one, even with such a large non-detection rate, across a range of parameters (e.g., redshift, column density, UV/radio luminosity, radio source size) marks an attempt to better test and constrain the multitude of factors that impact the gas reservoirs and will allow for a more comprehensive understanding of the physical, kinematic, and chemical properties of the neutral gas in the AGN environment.

Our low detection rate does not allow us to disentangle high (or even low) redshift biases against detection of $21 \mathrm{~cm}$ absorption. It is now clear that targeting UV- 
bright quasars, a condition imposed to reliably constrain the redshift of the source using the rest-frame UV continuum, are not identifying targets with large enough reservoirs of cold, neutral gas detectable with large radio surveys such as this study. Future surveys should be designed to target highly extincted, obscured sources that are not bright in the UV. Such surveys can leverage the infrared capabilities from WFIRST or even JWST to serendipitously detect potential HI targets that are UV/optical faint but bright in the mid-IR (e.g., Yan et al. 2012 ,2016). The SKA and its pathfinders will undoubtedly inaugurate a new era in HI absorption surveys, owing to large instantaneous bandwidth coverage and field of view. The improved sensitivity and spectral coverage achievable with the SKA will overcome observational biases introduced by the conventional requirement of an optical redshift, ushering in a future of true "blind" HI absorption surveys.

\section{CONCLUSIONS}

We present the results of the largest survey to date to search for intrinsic $\mathrm{HI} 21 \mathrm{~cm}$ and $\mathrm{OH} 18 \mathrm{~cm}$ absorption at redshifts between $0.02<z<3.8$ in 145 radio sources with the Green Bank Telescope. Below we summarize the major findings of this study.

1. Despite having the sensitivity to re-detect six previous detections of associated HI $21 \mathrm{~cm}$ absorption in our sample, we make no new detections of $21 \mathrm{~cm}$ absorption in the 108 sources for which we have RFI-free spectra. The overall HI $21 \mathrm{~cm}$ absorption detection rate is $5.6 \%$, but no detections are made for $z>0.7$. This is significantly lower than similar low redshift surveys.

2. We place an upper limit on the column density for all non-detections not affected by RFI, reaching a mean optical depth of $\tau_{3 \sigma}<0.023$. 79 of the radio sources have never been observed for $\mathrm{HI}$ $21 \mathrm{~cm}$ before. Despite low flux density values at the frequency of our redshifted $21 \mathrm{~cm}$ spectral observations, coupled with the significant presence of RFI in our low-frequency spectral observations, we reached adequate sensitivity to detect nearly any damped Ly $\alpha$ system.

3. We have searched 125 of our sources for intrinsic molecular $\mathrm{OH} 18 \mathrm{~cm}$ absorption. We do not detect any of the four $\mathrm{OH}$ lines in the $102 \mathrm{RFI}$-free sources for which we have usable $\mathrm{OH}$ data, obtaining exclusively upper limits for all measured $\mathrm{OH}$ column densities. We do not detect $\mathrm{OH}$ toward five of the known $21 \mathrm{~cm}$ absorption systems for which we have spectra coverage. Lack of $\mathrm{OH}$ absorption in these systems is not a result of insensitive measurements and we place upper limits on the $\mathrm{OH}$ column that are consistent with previously detected $\mathrm{OH}$ absorption systems.

4. We find no HI $21 \mathrm{~cm}$ absorption above an ultraviolet luminosity of $L_{\mathrm{UV}} \gtrsim 10^{23} \mathrm{~W} \mathrm{~Hz}^{-1}$. This supports the hypothesis that intrinsic $21 \mathrm{~cm} \mathrm{ab-}$ sorption cannot be detected in galaxies when the UV luminosity exceeds this UV luminosity threshold (Curran et al. 2008). Lack of $21 \mathrm{~cm}$ absorption
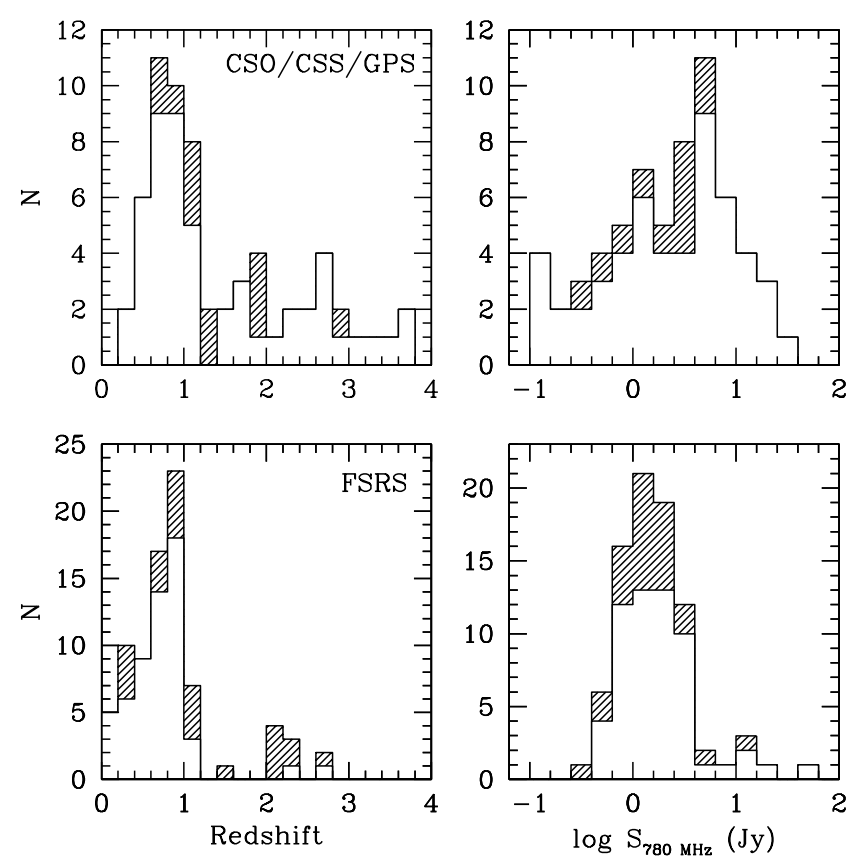

Figure 1. Redshift (left) and continuum flux density at the expected redshifted $21 \mathrm{~cm}$ line frequency (right) for the CSO, CSS, and GPS sources (top row) and the FSRSs (bottom row) in our sample. The hatched cells show the objects that could not be observed for spectral lines due to RFI.

in UV bright sources is an effect due to either the ionization of the neutral gas, higher spin temperatures in the environment around these luminous AGN, or sources that simply do not have a sufficient column of cold, neutral HI along the line of sight.

5. We do not find statistically significant evidence that the HI column density the of $21 \mathrm{~cm}$ systems in our sample depends on the radio jet size, UV luminosity, or radio $(1.4 \mathrm{GHz})$ luminosity. We are limited, however, by small numbers.

6. With the exception of a single source, all of our RFI-free sources beyond $z \sim 1.2$ arise in UVluminous sources above the UV luminosity threshold of $L_{\mathrm{UV}}=10^{23} \mathrm{~W} \mathrm{~Hz}^{-1}$. This suggests that a possible explanation that the dearth of intrinsic $21 \mathrm{~cm}$ absorption systems at high redshift is an selection bias favoring UV-luminous AGN. Requiring optical redshifts (rest-frame UV for $z \gtrsim 1.6$ ) imposes an optical bias and low-dust column, selecting the brightest, least obscured, and UV-bright radio sources at all redshifts. Such sources create an environment where neutral gas cannot survive and as such, are not detectable in $21 \mathrm{~cm}$ absorption. 

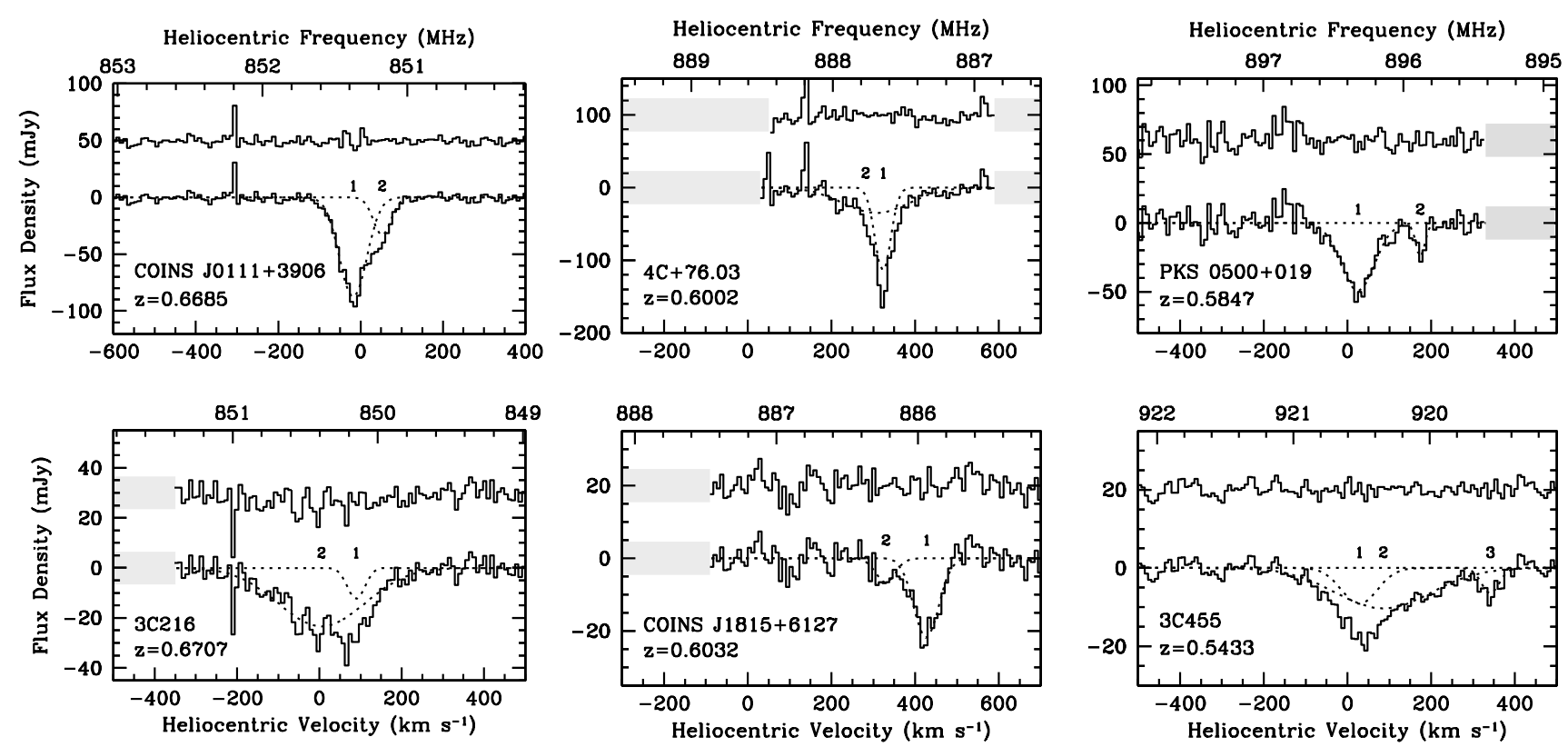

Figure 2. Intrinsic HI $21 \mathrm{~cm}$ absorption line systems. Dotted lines indicate Gaussian fits, numbered by component listed in Table 4 Upper spectra show the residual spectrum, offset for clarity. The velocity scale is in the rest frame of each object, defined by the optical heliocentric redshift of each radio source (Table 1). Spectral regions lost to radio frequency interference are greyed out.

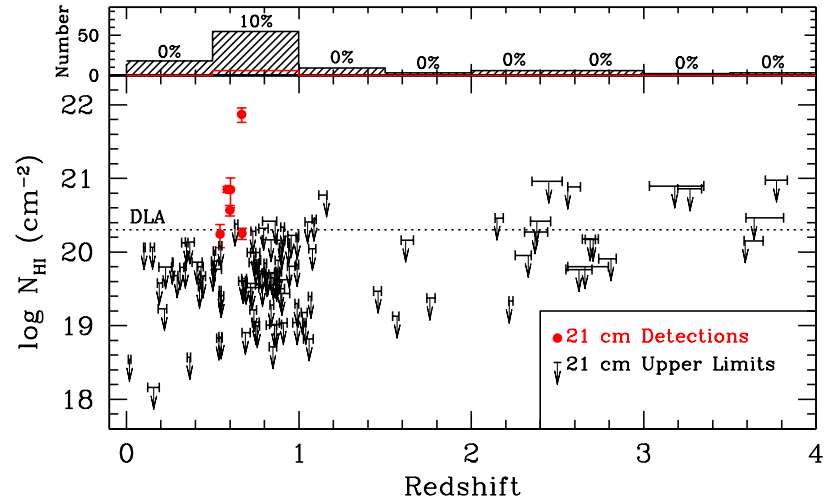

Figure 3. HI column density versus redshift. The lower panel shows either the detected HI column density at the redshift of the detected $21 \mathrm{~cm}$ absorption line or its $3 \sigma$ upper limit in the case of non-detections. The horizontal bar on the $21 \mathrm{~cm}$ non-detections indicates the redshift search region, and the downward-pointing arrow is centered on the systemic redshift of each object; the $21 \mathrm{~cm}$ line search regions are generally determined by RFI conditions. The solid red points represent sources detected in $21 \mathrm{~cm}$ absorption with the $1 \sigma$ error in the measured column density shown with the vertical error bars. The horizontal dotted line indicates the fiducial threshold for damped Ly $\alpha$ systems (DLAs; $N_{\mathrm{HI}} \geq 2 \times 10^{20} \mathrm{~cm}^{-2}$ ). The upper panel indicates the number of objects surveyed in each $\Delta z=0.5$ redshift bin (black, hatched histogram), the number of intrinsic HI $21 \mathrm{~cm}$ absorption lines detected (red histogram), and the $21 \mathrm{~cm}$ absorption fraction (text percentages above each bin).

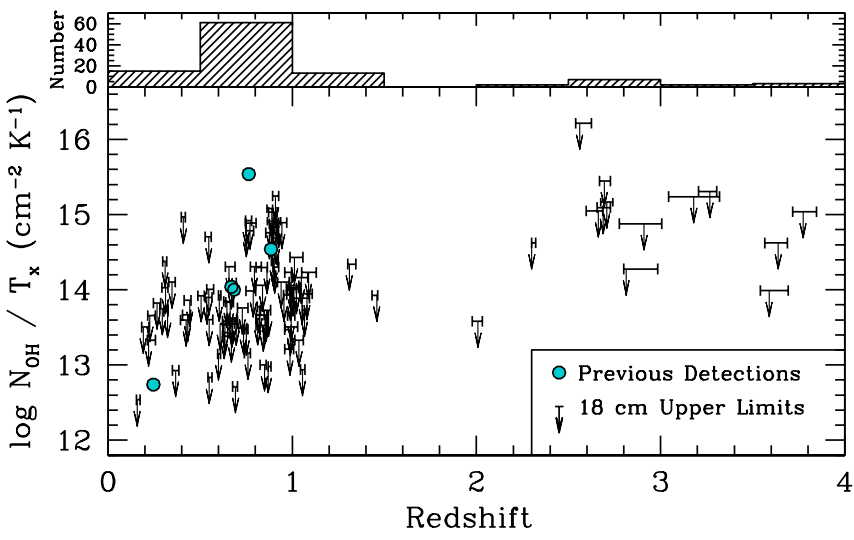

Figure 4. The lower panel shows the $3 \sigma$ upper limit $\mathrm{OH}$ column density versus the optical redshift. The horizontal bar indicates the redshift search region, typically determined by RFI conditions, and the downward-pointing arrow is centered on the systemic redshift of each object. The upper panel indicates the number of objects surveyed in each $\Delta z=0.5$ redshift bin (black, hatched). We show previous $\mathrm{OH} 1665 \mathrm{MHz}$ absorption detections as teal symbols. 


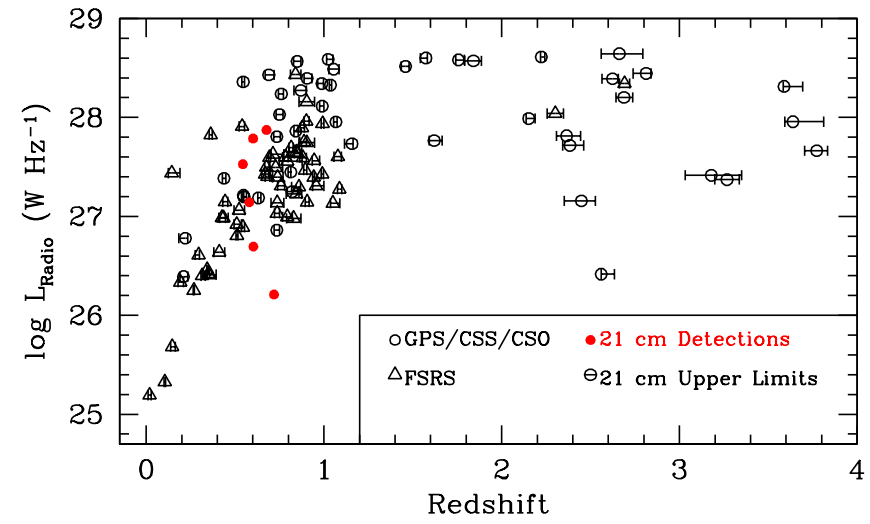

Figure 5. Radio luminosity versus redshift for RFI-free sources. We use the optical redshift for sources not detected in $21 \mathrm{~cm}$ and the absorption redshift for the six $21 \mathrm{~cm}$ detections. The horizontal bar on the $21 \mathrm{~cm}$ non-detections indicates the redshift search region for each source, generally determined by the RFI conditions. The solid red symbols represent $21 \mathrm{~cm}$ detections and unfilled black symbols represent the non-detections. The shape of the symbols represent the radio source identification: circles represent GPS, CSS, and CSO sources, and triangles represent FSRSs.

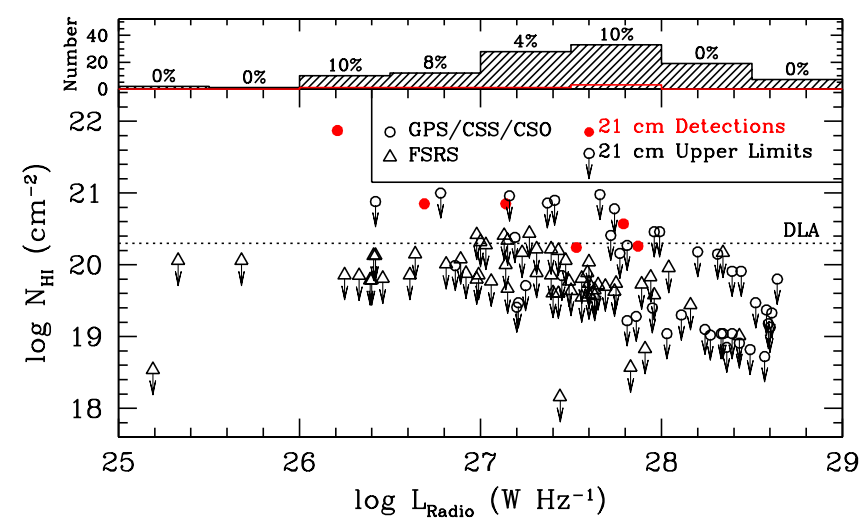

Figure 6. The lower panel shows the HI column density (upper limit in the case of non-detections) as a function of the specific radio luminosity. The horizontal dotted line indicates the canonical threshold for DLA systems. The solid red symbols represent $21 \mathrm{~cm}$ detections and unfilled symbols represent the non-detections. The shape of the symbols represent the radio source identification: circles represent GPS, CSS, and CSO sources, and triangles represent FSRSs. The upper panel indicates the total number of objects surveyed in each $\Delta L_{\text {Radio }}=0.5$ dex bin (black, hatched histogram), the number of intrinsic HI $21 \mathrm{~cm}$ absorption lines detected (red histogram), and the $21 \mathrm{~cm}$ absorption fraction (text percentages above each bin).

We are grateful for the valuable comments on this work by an anonymous referee that improved the scientific outcome and quality of the paper. The authors thank T. Yan and K. Willett for data reduction scripts. The authors thank the staff members at the Green Bank Telescope for their assistance and support. This research has made use of the NASA/IPAC Extragalactic Database (NED) which is operated by the Jet Propulsion Laboratory, California Institute of Technology, under contract with NASA. This publication makes use of data products from the Two Micron All Sky Survey, which is a joint project of the University of Massachusetts and the Infrared Processing and Analysis Center/California Institute of Technology, funded by the National Aeronautics and Space Administration and the National Science

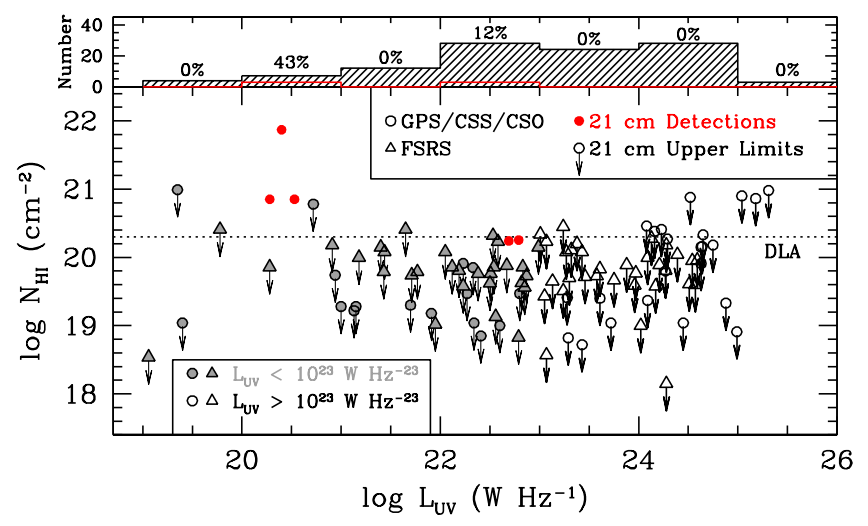

Figure 7. HI column density $\mathrm{N}_{\mathrm{HI}}$ (upper limit for sources not detected in $21 \mathrm{~cm}$ absorption) as a function of the ultraviolet luminosity (Section 5.2). The horizontal dotted line indicates the threshold for DLAs. The solid red symbols represent $21 \mathrm{~cm}$ detections and downward pointing arrows are the upper limits for non-detections. Filled gray symbols represent the non-detections that are below the UV threshold of $10^{23} \mathrm{~W} \mathrm{~Hz}^{-1}$ and open symbols represent the non-detections that are above the UV threshold. The shape of the symbols represent the radio source identification: circles represent GPS, CSS and CSO sources and triangles represent FSRSs. The upper panel indicates the total number of objects surveyed in each $\Delta L_{\mathrm{UV}}=1$ dex luminosity bin (black, hatched histogram), the number of intrinsic HI $21 \mathrm{~cm}$ absorption lines detected (red histogram), and the $21 \mathrm{~cm}$ absorption fraction (text percentages above each bin). We confirm the findings of Curran et al. (2008) that $21 \mathrm{~cm}$ absorption is not detected in sources with a UV luminosity above the threshold of $10^{23} \mathrm{~W} \mathrm{~Hz}^{-1}$. However, we detect no new absorbers in the sample below this UV luminosity threshold.

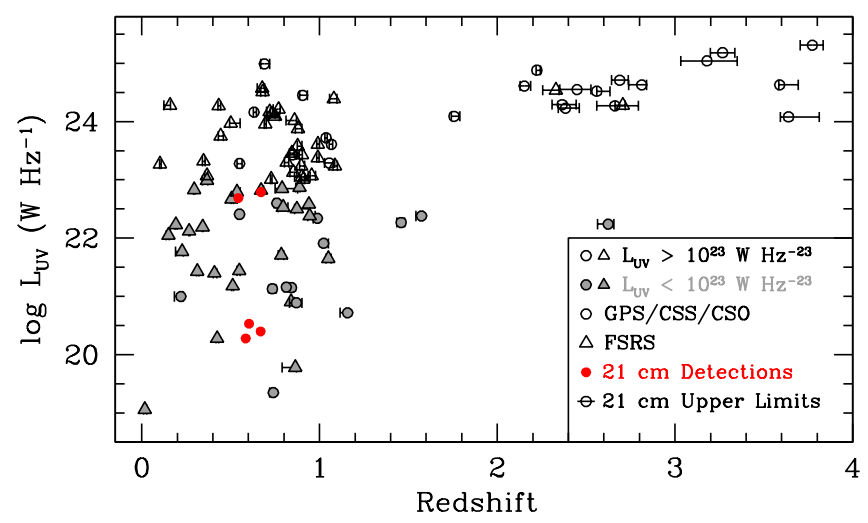

Figure 8. UV luminosity versus redshift for our RFI-free sources. We use the optical redshift for sources not detected in $21 \mathrm{~cm}$ and the absorption redshift for the five $21 \mathrm{~cm}$ detections with a measured UV luminosity. The horizontal bar on the $21 \mathrm{~cm}$ nondetections indicates the redshift search region for each source, generally determined by the RFI conditions. The solid red symbols represent $21 \mathrm{~cm}$ detections and unfilled black symbols represent the non-detections. The shape of the symbols represent the radio source identification: circles represent GPS, CSS, and CSO sources and triangles represent FSRSs. $21 \mathrm{~cm}$ absorption systems have never been detected in sources with a UV luminosity above a threshold of $10^{23} \mathrm{~W} \mathrm{~Hz}^{-1}$. This figure demonstrates the difficulty in selecting sources below the UV luminosity threshold (filled gray symbols) at redshifts above $z \sim 1$ that may possibly host $21 \mathrm{~cm}$ absorbers. 


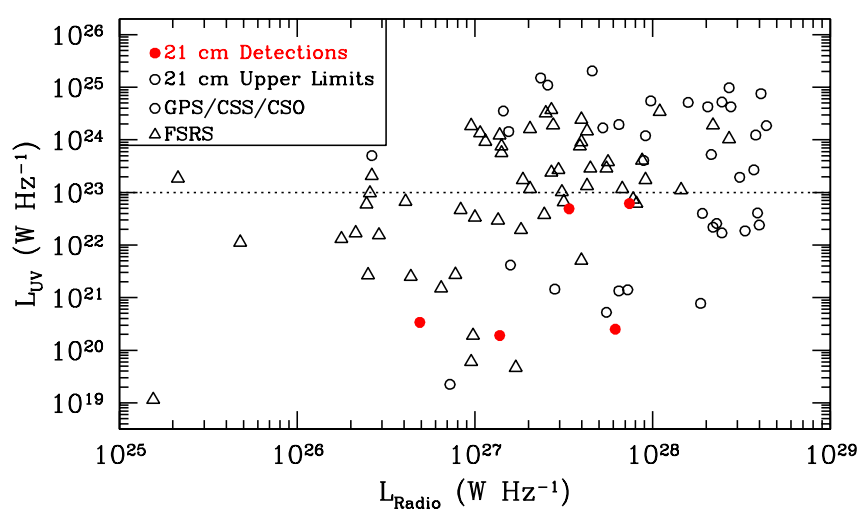

Figure 9. Ultraviolet luminosity versus radio continuum luminosity at the frequency of the redshifted $21 \mathrm{~cm}$ line. The solid red symbols represent $21 \mathrm{~cm}$ detections and unfilled black symbols represent non-detections. The shape of the symbols represent the radio source identification: circles represent GPS, CSS, and CSO sources and triangles represent FSRSs. The horizontal dotted line indicates the UV luminosity threshold $\left(L_{\mathrm{UV}}=10^{23} \mathrm{~W} \mathrm{~Hz}^{-1}\right)$ above which $21 \mathrm{~cm}$ absorption has not been detected.

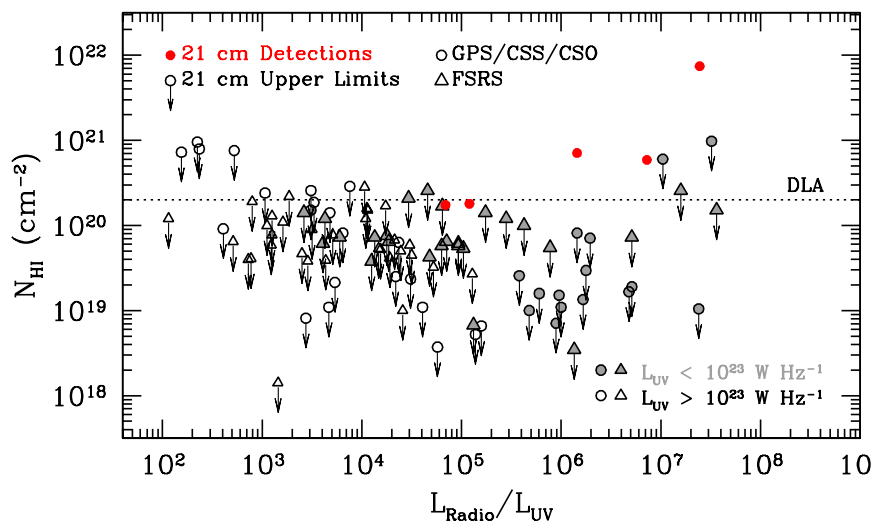

Figure 10. HI column density (upper limit for sources not detected in $21 \mathrm{~cm}$ absorption) versus the ratio of the radio to the UV luminosity (or flux density). The filled gray points represent HI $21 \mathrm{~cm}$ non-detections below the intrinsic UV luminosity threshold of $10^{23} \mathrm{~W} \mathrm{~Hz}^{-1}$ and black open symbols represent sources with an UV luminosity above the threshold. The solid red symbols represent $21 \mathrm{~cm}$ detections. The shape of the symbols represent the radio source identification: circles represent GPS, CSS, and CSO sources and triangles represent FSRSs. The horizontal dotted line indicates the threshold for damped Ly $\alpha$ systems

Foundation. Parts of this research were supported by the Australian Research Council Centre of Excellence for All Sky Astrophysics in 3 Dimensions (ASTRO 3D), through project number CE170100013. The authors acknowledge the invaluable labor of the maintenance and clerical staff at their institutions, whose contributions make scientific discoveries a reality. KG acknowledges the staff at the University House for the supportive and collaborative writing environment.

Facilities: GBT

Software: GBTIDL (Marganian et al. 2006)

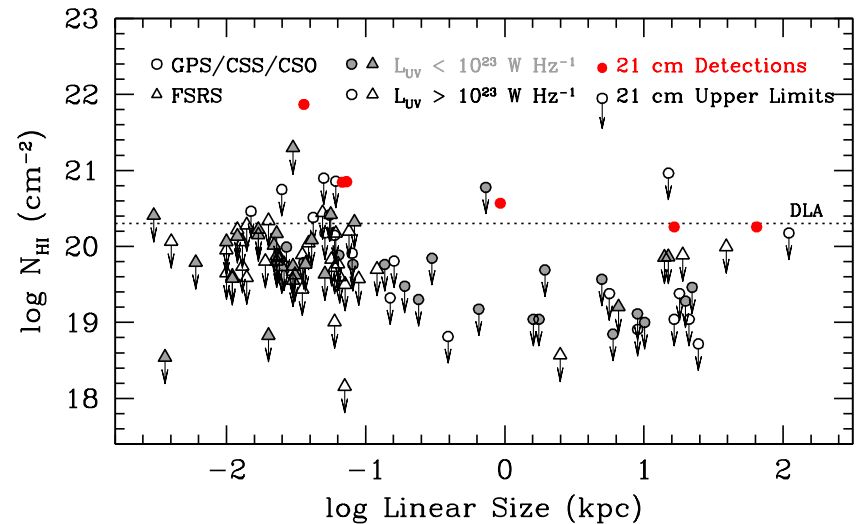

Figure 11. HI column density (upper limit for sources not detected in HI) versus linear size of the radio source. The solid red symbols represent $21 \mathrm{~cm}$ detections, the filled gray data points represent non-detections with an intrinsic UV luminosity below the UV threshold, and black open symbols represent non-detections with a UV luminosity above the $10^{23} \mathrm{~W} \mathrm{~Hz}^{-1}$ threshold. The shape of the symbols represent the radio source identification: circles represent GPS, CSS, and CSO sources and triangles represent FSRSs. The horizontal dotted line indicates the threshold for damped Ly $\alpha$ systems.

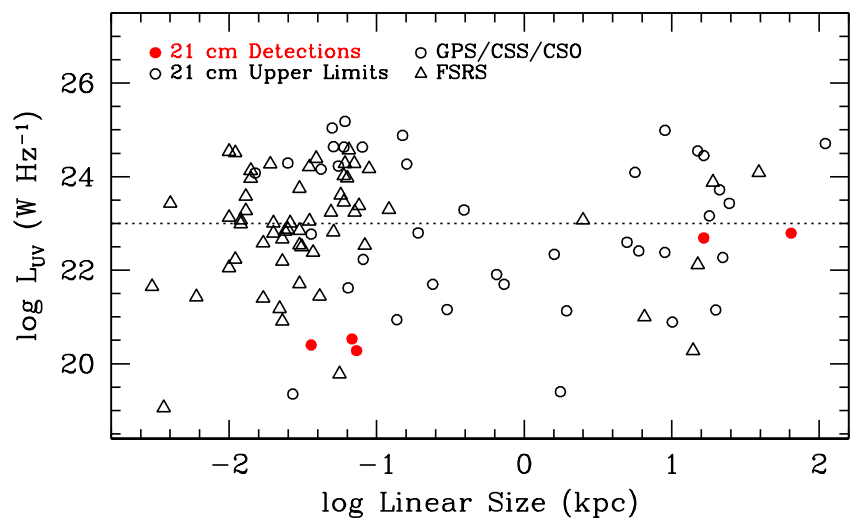

Figure 12. Ultraviolet luminosity versus linear size of the radio sources. The solid red symbols represent $21 \mathrm{~cm}$ detections and black symbols represent the upper limits to the column density for non-detections. The shape of the symbols represent the radio source identification: circles representing GPS, CSS, and CSO sources and triangles represent FSRSs. The horizontal dotted line indicates the UV luminosity threshold of $\left(L_{\mathrm{UV}}>10^{23} \mathrm{~W} \mathrm{~Hz}^{-1}\right)$.

\section{REFERENCES}

Adelman-McCarthy, J. K., Agüeros, M. A., Allam, S. S. et al. 2008, ApJS, 175, 297

Aditya, J. N .H. S., Kanekar, N., \& Kurapati, S. 2016, MNRAS, 455,5000

Aditya, J. N .H. S., Kanekar, N., Prochaska, J. X., et al. 2017, MNRAS, 465, 5011

Aditya, J. N. H. S. \& Kanekar, N. 2018a, MNRAS, 473, 59

Aditya, J. N. H. S. \& Kanekar, N. 2018b, MNRAS, 481, 1578

Allen, D. A., Ward, M. J., \& Hyland, A. R. 1982, MNRAS, 199, 969

Antonucci, R. R. J., \& Ulvestad, J. S. 1985, ApJ, 294, 158

Atlee, D. W. \& Gould, A. 2007, ApJ, 664, 53

Augusto, P., Gonzalez-Serrano, J. I., Perez-Fournon, I., \& Wilkinson, P. N. 2006, MNRAS, 368, 1411

Bajtlik, S., Duncan, R. C., Ostriker, J. P., 1988, ApJ, 327, 570

Bessell, M. S. 1979, PASP, 91, 589

Campins, H., Rieke, G. H., \& Lebofsky, M. J. 1985, AJ, 90, 896

Carballo, R., González-Serrano, J. I, Benn, C. R., Sánchez, S. F., \& Vigotti, M. 1999, MNRAS, 306, 137 


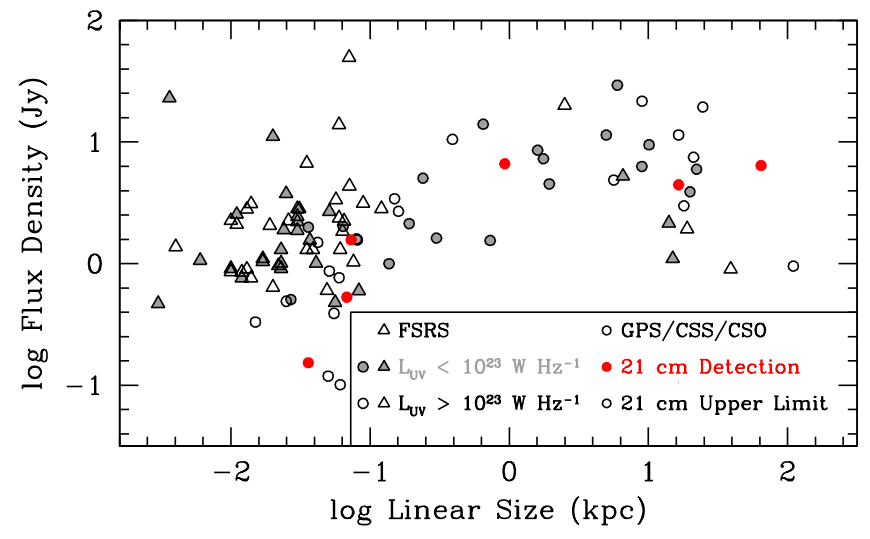

Figure 13. Continuum flux density (Jy) as a function of the linear size of the radio emission structures in the sample. The solid red symbols represent $21 \mathrm{~cm}$ detections and unfilled black symbols represent non-detections. The shape of the symbols indicates the radio source identification: circles represent GPS, CSS, and CSO sources and triangles represent FSRSs.

Carilli, C. L., Menten, K. M., Reid, M. J., Rupen, M. P., \& Yun, M. S. 1998, ApJ, 498, 175

Chen, P. S., Fu, H. W., \& Gao, Y. F. 2005, NewA, 11, 27

Chengalur, J. N., de Bruyn, A. G., \& Narasimha, D. 1999, ASPC, 156,228

Chengalur, J. N. \& Kanekar, N. 2003, Phys. Rev. Lett., 91, 241302

Chun, M. R., Gharanfoli, S., Kulkarni, V. P., \& Takamiya, M. 2006, AJ, 131, 686

Cody, A. M. \& Braun, R. 2009, A\&A, 400, 871

Condon, J. J., Hicks, P. D, \& Jauncey, D. L. 1977, AJ, 82, 692

Condon, J. J., Cotton, W. D., Greisen, E. W., \& Yin, Q. F. 1998, AJ, 115,1693

Cowie, L. L. \& Songaila, A. 1995, ApJ, 453, 596

Curran, S. J., Whiting, M. T., Murphy, M. T. 2006, MNRAS, 371,431

Curran, S. J., Whiting, M. T., Wiklind, T., Webb, J. K, Murphy, M. T., \& Purcell, C. R. 2008, MNRAS, 391, 765

Curran, S. J. \& Whiting, M. T. 2010, ApJ, 712, 303

Curran, S. J. 2010, MNRAS, 402, 2647

Curran, S. J., Whiting, M. T., Murphy, M. T., et al. 2011, MNRAS, 413, 1165

Curran, S. J., Whiting, M. T., Sadler, E. M., \& Bignell, C. 2013, MNRAS, 428, 2053

Curran, S. J., Duchesne, S. W., Divoli, A., \& Allison, J. R. 2016, MNRAS, 462, 4197

Curran, S. J., Hunstead, R. W., Johnston, H. M., et al. 2017, MNRAS, 470,4600

Curran, S. J. 2018, PASA, 35, 36

Curran, S. J., Hunstead, R. W., Johnston, H. M., et al. 2019, MNRAS, 484, 1182

Darling, J. 2003, Phys. Rev. Lett., 91, 011301

Darling, J. 2004, ApJ, 612, 58

de Vries, W. H., Barthel, P. D., \& O'Dea, C. P. 1997, A\&A, 321, 105

de Vries, W. H., O'Dea, C. P., Baum, S. A., et al. 1997b, ApJS, 110, 191

de Vries, W. H., O'Dea, C. P., Perlman, E., Baum, S. A., Lehnert, M. D., Stocke, J., Rector, T., \& Elston, R. 1998, ApJ, 503, 138 de Vries, W. H., O'Dea, C. P., Barthel, P. D., et al. 2000, ApJ, 120,2300

Douglas, J. N., Bash, F. N., \& Bozyan, F. A. 1996, AJ, 111, 1954

Drake, C. L., McGregor, P. J., \& Dopita, M. A. 2004, AJ, 128, 955

Drinkwater, M. J., Webster, R. L., Francis, P. J., et al. 1997, MNRAS, 284, 85

Elitzur, M. 1992, Astronomical Masers (Dordrecht: Kluwer); http://adsabs.harvard.edu/abs/1992ASSL..170.....E

Ellison, S. L., Hall, P. B., \& Lira, P. 2005, AJ, 130, 1345

Elvis, M., Wilkes, B. J., McDowell, J. C., et al. 1994, ApJS, 95, 1

Fanti, C., Fanti, R., Parma, P., et al. 1989, A\&A, 217, 44

Fanti, R., Fanti, C., Schilizzi, R. T., et al. 1990, A\&A, 231, 333

Fanti, C., Pozzi, F., Dallacasa, D., et al. 2001, A\&A, 369, 380
Fey, A. L. \& Charlot, P. 1997, ApJS, 111, 95

Fey, A. L. \& Charlot, P. 2000, ApJS, 128, 17

Ficarra, A., Grueff, G., \& Tomassetti, G. 1985, A\&AS, 59, 255

Field, G. B. 1959, ApJ, 129, 536

Fiorucci, M., Ciprini, S., \& Tosti, G. 2004, A\&A, 419, 25

Fomalont, E. B., Frey, S., Paragi, Z., et al. H. 2000, ApJS, 131, 95

Francis, P. J., Whiting, M. T., \& Webster, R. L. 2000, PASP, 17, 56

Fugmann, W. 1988, A\&A, 205, 86

Gardner, F. F. \& Whiteoak, J. B. 1978, MNRAS, 183, 711

Gelderman, R. \& Whittle, M., 1994, ApJS, 91, 491

Geréb, K., Morganti, R., \& Oosterloo, T. A. 2014, A\&A, 569, A35

Geréb, K., Maccagni, F. M., Morganti, R., \& Oosterloo, T. A. 2015, A\&A, 575, 44

Glass, I. S. 1981, MNRAS, 194, 795

Godwin, J. G. Bucknell, M. J., Dixon, K. L., Green, M. R., Peach, J. V., \& Wallis, R. E. 1977, Obs, 97, 238

Gupta, N., Salter, C. J., Saikia, D. J., Ghosh, T., \& Jeyakumar, S. 2006, MNRAS, 373, 972

Gurvits, L. I., Kellermann, K. I., \& Frey, S. 1999, A\&A, 342, 378

Hambly, N., MacGillivray, H. T., Read, M. A., et al. 2001, MNRAS, 326, 1279

Healey, S. E., Romani, R. W., Taylor, G. B., et al. 2007, ApJS, 171,61

Healey, S. E., Romani, R. W., Cotter, G., et al. 2008, ApJS, 175, 97

Hyland, A. R. \& Allen, D. A. 1982, MNRAS, 199, 943

Ishwara-Chandra, C. H., Dwarakanath, K. S. \& Anantharamaiah, K. R. 2003, JA\&A, 24, 37

Jackson, C. A., Wall, J. V., Shaver, P. A., Kellermann, K. I., Hook, I. M., \& Hawkins, M. R. S. 2002, A\&A, 386, 97

Jones, T. W., O'Dell, S. L., \& Stein, W. A. 1974, ApJ, 192, 261

Jorgenson, R. A., Wolfe, A. M., Prochaska, J. X., et al. 2006, ApJ, 646, 730

Kanekar, N., Chengalur, J. N., de Bruyn, A. G., \& Narasimha, D. 2003, MNRAS, 345, 7

Kanekar, N. \& Chengalur, J. N. 2003, A\&A, 399, 857

Kanekar, N. \& Chengalur, J. N. 2004, MNRAS, 350, 17

Kanekar, N., Carilli, C. L., Langston, G. I., et al. 2005, Phys.

Rev. Lett., 95, 261301

Kanekar, N., Subrahmanyan, R., Ellison, S. L., et al. 2006, MNRAS, 370, L46

Kanekar, N., Langston, G. I., Stocke, J. T., Carilli, C. L. \& Menten, K. M. 2012, ApJ, 746, L16

Kanekar, N., Prochaska, J. X., Smette, A., et al. 2014, MNRAS, 438,2131

Kanekar, N., Ghosh, T., \& Chengalur, J. N. 2018, Phys. Rev. Lett., 120, 061302

Kellermann, K. I., Vermeulen, R. C., Zensus, J. A., \& Cohen, M. H. 1998, AJ, 115, 1295

Komatsu, E., Smith, K. M., Dunkley, J., et al. 2011, ApJS, 192, 18

Kotilainen, J. K., Hyvönen, T., \& Falomo, R. 2005, A\&A, 440, 831

Kuhn, O. P. 2004, MNRAS, 348, 647

Labiano, A., Barthel, P. D., O’Dea, C. P., de Vries, W. H., Pérez, I., \& Baum, S. A. 2007, A\&A, 463, 97

Laing, R. A. \& Peacock, J. A. 1980, MNRAS, 190, 903

Large, M. I., Mills, B. Y., Little, A. G., Crawford, D. F., \& Sutton, J. M. 1981, MNRAS, 194, 693

Lister, M. L. \& Homan, D. C. 2005, AJ, 130, 1389

Maccagni, F. M., Morganti, R., Oosterloo, T. A., Geréb, K., \& Maddox, N. 2017, A\&A, 604, A43

Marganian, P., Garwood, R. W., Braatz, J. A., Radziwill, N. M., \& Maddalena, R. J. 2006, ASPC, 351, 512

Matthews, T. A. \& Sandage, A. R. 1963, ApJ, 138, 30

McAlary, C. W., McLaren, R. A., McGonegal, R. J. \& Maza, J.

1983, ApJS, 52, 341

Menon, T. K. 1983, AJ, 88, 598

Molina, M., Landi, R., Bassini, et al. 2012, A\&A, 548, 32

Moore, C. B., Carilli, C. L., \& Menten, K. M. 1999, ApJ, 510, L87

Morganti, R., Killeen, N. E. B., \& Tadhunter, C. N., 1993,

MNRAS, 263, 1023

Morganti, R., Oosterloo, T. A., Reynolds, J. E., \& Tadhunter, C. N. 1997, MNRAS, 284, 541

Morganti, R., Oosterloo, T. A., Tadhunter, C. N., van Moorsel, G., Killeen, N., \& Wills, K. A. 2001, MNRAS, 323, 331 
Moss, V. A., Allison, J. R., Sadler, E. M., et al. 2017, MNRAS, 471,2952

Murphy, D. W., Browne, I. W. A., \& Perley, R. A. 1993, MNRAS, 264, 298

Murphy, M. T., Webb, J. K., Flambaum, V. V., et al.2001, MNRAS, 327, 1208

O'Dea, C. P., Baum, S. A., \& Stanghellini, C. 1991, ApJ, 380, 660

O'Dea, C. P. \& Baum, S. A. 1997, ApJ, 113, 148

O'Dell, S. L., Puschell, J. J., Stein, W. A., \& Warner, J. W. 1978, ApJS, 38, 267

Odell, S. L., Puschell, J. J., Stein, W. A., et al. 1978a, ApJ, 224, 22

Ojha, R., Zacharias, N., Hennessy, G. S., Gaume, R. A., \& Johnston, K. J. 2009, AJ, 138, 845

Orienti, M., Dallacasa, D., \& Stanghellini, C. 2007, A\&A, 475, 813

Owsianki, I. \& Conway, J. E. 1998, A\&A, 337, 69

Peacock, J. A. \& Wall, J. V. 1982, MNRAS, 198, 843

Peck, A. B. \& Taylor, G. B. 2000, ApJ, 534, 90

Petrov, L., Kovalev, Y. Y., Fomalont, E. B., \& Gordon, D. 2008, AJ, 136, 580

Pihlström, Y. M., Conway, J. E., \& Vermeulen, R. C. 2003, A\&A, 404, 871

Rahmani, H., Srianand, R., Gupta, N., et al. 2012, MNRAS, 425, 556

Ramírez, A., de Diego, J. A, Dultzin-Hacyan, D., \& González-Pérez, J. N. 2004, A\&A, 421, 83

Rao, S. A., Turnshek, D. A, \& Nestor, D. B. 2006, ApJ, 636, 610

Readhead, A. C. S, Taylor, G. B., Xu, W., Pearson, T. J.,

Wilkinson, P. N., \& Polatidis, A. G. 1996, ApJ, 460, 612

Rengelink, R .B, Tang, Y., de Bruyn, A. G., et al. 1997, A\&A, 124, 259

Rhee, J., Lah, P., Briggs, F. H., et al. 2018, MNRAS, 473, 1879

Roberts, M. S, 1970, ApJ, 161, L9

Sandage, A. 1965, ApJ, 141, 1560

Sandage, A., Philippe, V., \& Wyndham, J. D. 1965a, ApJ, 142, 1307

Sandage, A. \& Wyndham, J. D. 1965b, ApJ, 141, 328

Sbarufatti, B., Treves, A., Falomo, R., Heidt, J., Kotilainen, J., \& Scarpa, R. 2005, AJ, 129, 559

Sbarufatti, B.. Ciprini, S., Kotilainen, J., et al. 2009, AJ, 137, 337

Schmitt, H. R. \& Kinney, A. L. 1996, ApJ, 463, 498

Schneider, D. P., Gunn, J. E., \& Hoessel, J. G. 1983, ApJ, 264, 337

Shen, Z. -Q., Wan, T. -S., Moran, J. M., Jauncey, D. L., Reynolds, J. E., \& Tzioumis, A. K. 1997, AJ, 114, 1999

Shen, Z. -Q., Wan, T. -S., Moran, J. M., et al. 1998, AJ, 115, 1357

Simpson, C. \& Rawlings, S. 2000, MNRAS, 317, 1023

Skrutskie, M. F., Cutri, R. M., Stiening, R., et al. 2006, AJ, 131, 1163
Smith, E. P. \& Heckman, T. M. 1989, ApJS, 69, 365

Smith, J. A., Tucker, D. L., Kent, S., et al. 2002, AJ, 123, 2121

Snellen, A. G., Lehnert, M. D., Bremer, M .N., \& Schilizzi, R. T. 2002, MNRAS, 337, 981

Spencer, R. E., McDowell, J. C., Charlesworth, M., Fanti, C., Parma, P., \& Peacock, J. A. 1989, MNRAS, 240, 657

Srianand, R., Gupta, N., Petitjean, P., Noterdaeme, P., \& Ledoux, C. 2010, MNRAS, 405, 1888

Stanghellini, C., Baum, S. A., O'Dea, C. P., \& Morris, G. B. 1990, A\&A, 233, 379

Stanghellini, C., O'Dea, C. P., Baum, S. A., \& Laurikainen, E. 1993, ApJS, 88, 1

Stanghellini, C., O'Dea, C. P., Dallacasa, D., Baum, S. A., Fanti, R., \& Fanti, C. 1998, A\&AS, 131, 303

Stanghellini, C., O'Dea, C. P., Dallacasa, D., et al. 2005, A\&A, 443,891

Stickel, M. \& Kuehr, H. 1996, A\&AS, 115, 11

Tapia, S., Craine, E. R., \& Johnson, K. 1976, ApJ, 203, 291

Tzanavaris, P, Webb, J. T., Murphy, M. T., Flambaum, V. V., \& Curran, S. J. 2005, Phys. Rev. Lett, 95, 041301

Tzanavaris, P, Murphy, M. T., Webb, J. T., Flambaum, V. V., \& Curran, S. J. 2007, MNRAS, 374, 634

Uson, J. M., Bagri, D. S., \& Cornwell, T. J. 1991, Phys. Rev. Lett., 67, 3328

van Gorkom, J. H., Knapp, G. R., Ekers, R. D., Ekers, D. D., Laing, R. A., \& Polk, K. S. 1989, AJ, 97, 708

van Langevelde, H. J., van Dishoeck, E. F., Sevenster, M. N., \& Israel, F. P. 1995, ApJ, 448, L123

Vermeulen, R. C., Pihlström, Y. M., Tschager, W., et al. 2003, A\&A, 404, 861

White, R. L. \& Becker, R. H. 1992, ApJS, 79, 331

Wilkinson, P. N., Polatidis, A. G., Readhead, A. C. S., Xu, W., \& Pearson, T. J. 1994, ApJ, 432, L87

Wills, D. \& Lynds, R. 1978, ApJS, 36, 317

Wolfe, A. M. \& Burbidge, G. R. 1975, ApJ, 200, 548

Wright, A. E., Ables, J. G., \& Allen, D. A. 1983, MNRAS, 205, 793

Wright, E. L. 2006, PASP, 118, 117

Xiang, L, Dallacasa, D., Cassaro, P., Jiang, D., \& Reynolds, C. 2005, A\&A, 434, 123

Xu, W., Readhead, A. C. S., Pearson, T. J., Polatidis, A. G., \& Wilkinson, P. N. 1995, ApJS, 99, 297

Yan, T., Stocke, J. T., Darling, J., \& Hearty, F. 2012, AJ, 144, 124

Yan, T., Stocke, J. T., Darling, J., Momjian, E., Sharma, S., \& Kanekar, N. 2016, AJ, 151, 74

Observations of Intrinsic HI and OH Lines Toward Compact Radio Sources

\begin{tabular}{|c|c|c|c|c|c|c|c|c|c|}
\hline \multirow{2}{*}{ Source } & \multirow{2}{*}{$\begin{array}{c}\alpha \\
(\mathrm{J} 2000)\end{array}$} & \multirow{2}{*}{$\begin{array}{c}\delta \\
(\mathrm{J} 2000)\end{array}$} & \multirow[t]{2}{*}{$z$} & \multirow{2}{*}{$\begin{array}{c}\nu_{\mathrm{HI}} \\
(\mathrm{MHz})\end{array}$} & \multirow{2}{*}{$\begin{array}{c}\text { HI Time } \\
(\mathrm{sec})\end{array}$} & \multirow{2}{*}{$\begin{array}{c}\text { OH Time } \\
(\mathrm{sec})\end{array}$} & \multirow{2}{*}{ Class. } & \multicolumn{2}{|c|}{ References } \\
\hline & & & & & & & & Class. & Spec \\
\hline $0018+729$ & 002127.4 & +731241.9 & $0.821(2)$ & $780.0(9)$ & 1493 & 896 & GPS & 1 & \\
\hline LBQS $0106+0119$ & 010838.8 & +013500.3 & $2.099(5)$ & $472.1(8)$ & 564 & 541 & FSRS & 2 & \\
\hline COINS J0111+3906 & 011137.3 & +3906 28.1 & $0.66847(3)$ & $851.322(15)$ & 1800 & $\ldots$ & GPS,CSO & $1,3,4$ & 1 \\
\hline UM321 & 012528.8 & -000555.9 & $1.07481(15)$ & $684.60(5)$ & 298 & 265 & FSRS & 2 & \\
\hline $3 \mathrm{C} 43$ & 012959.8 & +233820.3 & 1.459 & 577.6 & 1773 & 1725 & CSS & $3,5,6,7$ & \\
\hline $0133+476$ & 013658.6 & +475129.1 & 0.859 & 764.1 & 293 & 290 & FSRS & 2,8 & \\
\hline $3 \mathrm{C} 48$ & 013741.3 & +330935.1 & 0.367 & 1038 & 378 & 366 & CSS & $3,5,6$ & \\
\hline $3 \mathrm{C} 49$ & 014109.2 & +135328.1 & 0.62 & 876.8 & 893 & $\ldots$ & CSS & $3,5,6,7$ & 2 \\
\hline $0138-097$ & 014125.8 & -092843.7 & 0.733 & 818.6 & 298 & 271 & FSRS & 2 & \\
\hline PKS 0201+113 & 020346.7 & +113445.4 & $3.6390(10)$ & $306.19(7)$ & 2377 & 2377 & GPS & 1 & \\
\hline $0212+735$ & 021730.8 & +734932.6 & 2.367 & 421.9 & 528 & 502 & FSRS & 2,8 & \\
\hline B2 $0218+357$ & 022105.5 & +355613.7 & $0.944(2)$ & $730.7(8)$ & 270 & 264 & FSRS & 2 & \\
\hline $3 \mathrm{C} 066 \mathrm{~A}$ & 022239.6 & +430207.8 & 0.444 & 982.7 & 299 & 286 & FSRS & 8 & \\
\hline $0221+067$ & 022428.4 & +06 5923.3 & 0.511 & 940.0 & 296 & 272 & FSRS & 2,8 & \\
\hline $4 C+34.07$ & 022610.3 & +342130.3 & $2.910(2)$ & $363.28(19)$ & 2388 & 1763 & CSS & $3,5,6$ & \\
\hline HB89 $0235+164$ & 023838.9 & +163659.3 & 0.940 & 732.2 & 281 & 273 & FSRS & 2,8 & \\
\hline PKS 0237-23 & $0240 \quad 08.2$ & -230915.8 & 2.223 & 440.71 & 1493 & 3284 & GPS & 1,3 & \\
\hline PKS $0239+108$ & $0242 \quad 29.2$ & +110100.7 & 2.694 & 384.5 & 322 & 309 & FSRS & 2 & \\
\hline B3 $0248+430$ & 025134.5 & +431515.8 & 1.31 & 614.9 & 1493 & $\cdots$ & GPS & 1,3 & 3 \\
\hline $0306+102$ & 030903.6 & +102916.3 & 0.863 & 761.4 & 297 & 256 & FSRS & 2,8 & \\
\hline NGC 1275 & 031948.2 & +413042.1 & $0.01765(4)$ & $1395.77(5)$ & 278 & $\ldots$ & FSRS & 2,8 & \\
\hline
\end{tabular}


Table 1 - Continued

\begin{tabular}{|c|c|c|c|c|c|c|c|c|c|}
\hline \multirow[t]{2}{*}{ Source } & \multirow{2}{*}{$\begin{array}{c}\alpha \\
(\mathrm{J} 2000)\end{array}$} & \multirow{2}{*}{$\begin{array}{c}\delta \\
(\mathrm{J} 2000)\end{array}$} & \multirow[t]{2}{*}{$z$} & \multirow{2}{*}{$\begin{array}{c}\nu_{\mathrm{HI}} \\
(\mathrm{MHz})\end{array}$} & \multirow{2}{*}{$\begin{array}{c}\text { HI Time } \\
(\mathrm{sec})\end{array}$} & OH Time & Class. & Refer & nces \\
\hline & & & & & & $(\mathrm{sec})$ & & Class. & Spec. \\
\hline $\mathrm{OE}+131$ & 032152.1 & +122114.0 & 2.662 & 387.88 & 1472 & 1790 & GPS,CSS & $3,5,6,7$ & \\
\hline PKS $0327-241$ & 032954.1 & -235708.8 & 0.895 & 748.6 & 278 & 285 & FSRS & 2 & \\
\hline $0336-019$ & 033930.9 & -014635.8 & 0.852 & 767.0 & 290 & 288 & FSRS & 2 & \\
\hline $0346-279$ & 034838.1 & -274913.6 & 0.991 & 712.4 & 297 & 263 & FSRS & 2 & \\
\hline $4 C+76.03$ & 041045.6 & +765645.3 & 0.5985 & 888.6 & 818 & 896 & GPS,CSS & $3,5,6,7$ & 2 \\
\hline $0420-014$ & 042315.8 & -012033.1 & $0.91609(18)$ & $741.30(7)$ & 278 & 297 & FSRS & 2 & \\
\hline $0422+004$ & 042446.8 & +003606.3 & 0.310 & 1084.3 & 266 & 288 & FSRS & 2,8 & \\
\hline $3 \mathrm{C} 119$ & 043236.5 & +413828.4 & 1.023 & 702.1 & 1790 & 1791 & CSS & $3,5,6,7$ & \\
\hline $0440-003$ & 044238.7 & -001743.4 & 0.844 & 769.3 & 274 & 289 & FSRS & 2 & \\
\hline PKS 0454-234 & 045703.2 & -232452.0 & 1.003 & 708.1 & 298 & 266 & FSRS & 2 & \\
\hline PKS $0457+024$ & 045952.1 & +022931.2 & 2.384 & 419.7 & 1791 & 1721 & GPS & 1,3 & \\
\hline $0458-020$ & $\begin{array}{lll}05 & 01 & 12.8\end{array}$ & -015914.3 & 2.286 & 432.3 & 680 & 621 & FSRS & 2,8 & \\
\hline PKS $0500+019$ & $\begin{array}{lll}05 & 0321.2\end{array}$ & +020304.7 & $0.58457(2)$ & $896.398(11)$ & 164 & & GPS & 1,3 & 1 \\
\hline $3 \mathrm{C} 138$ & 052109.9 & +163822.1 & 0.76 & 807.1 & 550 & 1790 & CSS & $3,5,6$ & 2 \\
\hline PKS $0528-250$ & 053008.0 & -250329.9 & 2.813 & 372.5 & 2651 & 1493 & GPS & 1 & \\
\hline PKS 0539-057 & 054138.1 & -054149.4 & $0.839(3)$ & $772.4(1.3)$ & 298 & 250 & FSRS & 2 & \\
\hline $3 \mathrm{C} 147$ & 054236.1 & +495107.2 & 0.55 & 916.39 & 250 & 1791 & CSS & $3,5,6$ & 2 \\
\hline B3 $0552+398$ & 055530.8 & +394849.2 & $2.365(5)$ & $422.1(6)$ & 1767 & 288 & GPS & 1 & \\
\hline $0605-085$ & 060759.7 & -083450.0 & 0.872 & 757.8 & 516 & 489 & FSRS & 2 & \\
\hline $0607-157$ & 060941.0 & -154240.7 & $0.3240(10)$ & $1072.8(8)$ & 263 & 149 & FSRS & 2 & \\
\hline HB89 0636+680 & 064204.3 & +675835.6 & $3.1800(10)$ & $339.81(8)$ & 1941 & 896 & GPS & 1 & \\
\hline B2 $0711+35$ & 071442.8 & +353439.8 & 1.62 & 542.1 & 2302 & 2293 & GPS & 1 & \\
\hline $0735+178$ & 073807.4 & +174219.0 & 0.424 & 996.5 & 298 & 381 & FSRS & 2,8 & \\
\hline $0736+017$ & 073918.0 & +013704.6 & $0.189410(9)$ & 1192 & 349 & 321 & FSRS & 2,8 & \\
\hline B2 $0738+31$ & $07 \quad 41 \quad 10.7$ & +311200.2 & $0.6314(16)$ & $870.7(9)$ & 1194 & 1742 & GPS & 1,3 & 2 \\
\hline PKS $0742+10$ & 074533.1 & +101112.7 & $2.624(3)$ & $391.9(3)$ & 1681 & 1681 & GPS & 1,3 & 5 \\
\hline PKS 0743-006 & 074554.1 & -004417.5 & 0.994 & 712.3 & 278 & 1182 & GPS & 1,3 & \\
\hline $3 \mathrm{C} 186$ & $0744 \quad 17.5$ & +375317.2 & $1.0670(19)$ & $687.2(6)$ & 1790 & 1790 & CSS & $3,5,6$ & \\
\hline PKS $0745+241$ & 074836.1 & +240024.1 & $0.4092(10)$ & $1008.0(7)$ & 271 & 245 & FSRS & 2,8 & \\
\hline $0748+126$ & 075052.0 & +123104.8 & 0.889 & 750.9 & 283 & 297 & FSRS & 8,9 & \\
\hline COINS J0753+4231 & 075303.3 & +423130.8 & $3.5892(8)$ & $309.51(5)$ & 2687 & 2687 & $\mathrm{CSO}$ & 4 & \\
\hline HB89 0754+100 & 075706.6 & +09 5634.9 & $0.2660(10)$ & $1122.0(9)$ & 232 & 390 & FSRS & 2,8 & \\
\hline 3C190 & 080133.5 & +141442.4 & $1.1944(12)$ & $647.3(4)$ & 528 & $\ldots$ & CSS & $3,5,6$ & 4 \\
\hline SBS $0804+499$ & 080839.7 & +495036.5 & $1.430(2)$ & $584.5(5)$ & 596 & 592 & FSRS & 2 & \\
\hline PKS $0823+033$ & 082550.3 & +030924.5 & 0.506 & 942.2 & 262 & 285 & FSRS & 8,8 & \\
\hline PKS $0823-223$ & 082601.6 & -223027.2 & 0.910 & 742.7 & 298 & 283 & FSRS & 9 & \\
\hline $0828+493$ & 083223.2 & +491321.0 & 0.548 & 916.6 & 256 & 292 & FSRS & 2 & \\
\hline $0836+710$ & 084124.7 & +705342.2 & $2.1720(10)$ & $447.80(14)$ & 291 & 260 & FSRS & 2,8 & \\
\hline $\mathrm{OJ}+287$ & 085448.9 & +200630.6 & 0.306 & 1087.6 & 270 & $\ldots$ & FSRS & 2,8 & \\
\hline PKS $0858-279$ & 090040.0 & -280820.3 & 2.152 & 450.6 & 400 & $\ldots$ & GPS & 1 & \\
\hline TXS $0902+490$ & 090527.5 & +485050.0 & $2.6887(11)$ & $385.07(11)$ & 1433 & 1493 & GPS & 1 & \\
\hline $0906+015$ & $\begin{array}{lll}09 & 09 & 10.1\end{array}$ & +012135.6 & $1.020(3)$ & $701.8(1.1)^{\prime}$ & 290 & 285 & FSRS & 2,8 & \\
\hline $3 \mathrm{C} 216$ & 090933.5 & +425346.5 & $0.6703(12)$ & $850.4(6)$ & 1492 & 3581 & CSS & 5,6 & 2 \\
\hline $0917+449$ & 092058.5 & +444154.0 & $2.1899(2)^{\prime}$ & $445.28(3)$ & 577 & 369 & FSRS & 2,8 & \\
\hline $0919-260$ & 092129.4 & -261843.4 & $2.300^{\circ}$ & 429.4 & 597 & 592 & FSRS & 2 & \\
\hline $0923+392$ & 092703.0 & +390220.9 & $0.6952(3)$ & $837.90(15)$ & 298 & 274 & FSRS & 2 & \\
\hline $0925-203$ & 092751.8 & -203451.2 & $0.34741(15)$ & 1053.71(2) & 298 & 526 & FSRS & 2 & \\
\hline HB89 $0954+556$ & 095738.2 & +552257.8 & $0.896(8)$ & $749(3)$ & 268 & 284 & FSRS & 2 & \\
\hline HB89 $0954+658$ & $0958 \quad 47.2$ & +653354.8 & 0.368 & 1037 & 292 & & FSRS & 2 & \\
\hline $1004+141$ & 100741.5 & +135629.6 & $2.707(5)$ & $383.2(5)$ & 519 & 517 & FSRS & 2,8 & \\
\hline $3 \mathrm{C} 237$ & 100800.0 & +073016.4 & 0.87 & 759.6 & 1785 & 1771 & CSS & $3,5,6$ & \\
\hline PKS 1034-293 & $1037 \quad 16.1$ & -293402.8 & 0.312 & 1082 & 582 & 562 & FSRS & 2 & \\
\hline $1055+018$ & 105829.6 & +013358.8 & $0.888(5)$ & $752(2)$ & 243 & 266 & FSRS & 2,8 & \\
\hline $1116+128$ & 111857.3 & +123441.7 & $2.129(2)$ & $454.0(3)$ & 567 & 542 & FSRS & 2,8 & \\
\hline PKS 1124-186 & 112704.4 & $-1857 \quad 17.4$ & 1.050 & 691.9 & 249 & 289 & FSRS & 2,9 & \\
\hline B3 $1144+402$ & 114658.3 & +395834.3 & $1.0880(10)$ & $680.3(3)$ & 262 & 296 & FSRS & 2,8 & \\
\hline FBQS J1159+2914 & 115931.8 & +291443.8 & $0.7245(11)$ & $823.7(5)$ & 298 & 291 & FSRS & 2,8 & 2 \\
\hline $1202-262$ & 120533.2 & -263404.5 & 0.789 & 794.0 & 291 & 286 & FSRS & 2 & \\
\hline B2 $1219+28$ & 122131.7 & +281358.5 & 0.102 & 1288 & 596 & $\ldots$ & FSRS & 2 & \\
\hline $1222+037$ & 122452.4 & +033050.3 & $0.9556(11)$ & $726.3(4)$ & 261 & 290 & FSRS & 2,8 & \\
\hline PG $1222+216$ & 122454.5 & +212246.4 & $0.4320(10)$ & $991.9(7)$ & 299 & 273 & FSRS & 2,8 & \\
\hline B2 $1225+36$ & 122758.7 & +363511.8 & $1.973(2)$ & $477.8(3)$ & 300 & $\ldots$ & GPS,CSS & $1,3,5,6$ & \\
\hline $3 \mathrm{C} 273$ & 122906.7 & +020308.6 & $0.15834(7)$ & $1226.24(7)$ & 595 & 562 & FSRS & 2,8 & \\
\hline COINS J1244+4048 & 124449.2 & +404806.2 & $0.813(2)$ & $783.5(9)$ & 442 & 2239 & $\mathrm{CSO}$ & 4 & \\
\hline $3 \mathrm{C} 279$ & 125611.2 & -054721.5 & $0.5362(14)$ & $924.6(2)$ & 269 & 275 & FSRS & 2 & \\
\hline $1308+326$ & 131028.7 & +322043.8 & 0.996 & 711.6 & 228 & 298 & FSRS & 2,8 & \\
\hline $3 \mathrm{C} 287$ & 133037.7 & +250910.9 & 1.055 & 691.2 & 1790 & 1770 & CSS & $3,5,6$ & \\
\hline $3 \mathrm{C} 286$ & 133108.3 & +303033.0 & $0.8494(11)$ & $768.0(5)$ & 1194 & 1194 & CSS & $3,5,6$ & \\
\hline HB89 1333+459 & 133522.0 & +454238.2 & $2.4490(10)$ & $411.83(12)$ & 1750 & $\ldots$ & GPS & 1 & \\
\hline $1354+195$ & 135704.4 & +191907.4 & 0.720 & 824.8 & 274 & $\ldots$ & FSRS & 2,8 & \\
\hline B3 $1355+441$ & 135740.6 & +435359.8 & 0.646 & 862.9 & 844 & 1762 & GPS,CSO & 1,4 & 2 \\
\hline $1418+546$ & 141946.6 & +542314.8 & $0.1526(3)$ & $1232.4(3)$ & 272 & 275 & FSRS & 2,8 & \\
\hline 3C 305.1 & 144709.6 & +765621.8 & 1.132 & 666.2 & 895 & & CSS & $3,5,6$ & \\
\hline 3C309.1 & 145907.6 & +714019.9 & 0.905 & 745.6 & 896 & 865 & CSS & $3,5,6$ & \\
\hline $1504-166$ & 150704.8 & -165230.3 & $0.876(4)$ & $757.2(1.6)$ & 281 & & FSRS & 2 & \\
\hline
\end{tabular}


Table 1 - Continued

\begin{tabular}{|c|c|c|c|c|c|c|c|c|c|}
\hline \multirow[t]{2}{*}{ Source } & \multirow{2}{*}{$\begin{array}{c}\alpha \\
(\mathrm{J} 2000)\end{array}$} & \multirow{2}{*}{$\begin{array}{c}\delta \\
(\mathrm{J} 2000)\end{array}$} & \multirow[t]{2}{*}{$z$} & \multirow{2}{*}{$\begin{array}{c}\nu_{\mathrm{HI}} \\
(\mathrm{MHz})\end{array}$} & \multirow{2}{*}{$\begin{array}{c}\text { HI Time } \\
(\mathrm{sec})\end{array}$} & \multirow{2}{*}{$\begin{array}{l}\text { OH Time } \\
\quad(\mathrm{sec})\end{array}$} & \multirow{2}{*}{ Class. } & \multicolumn{2}{|c|}{ References } \\
\hline & & & & & & & & Class. & Spec. \\
\hline $3 \mathrm{C} 318$ & 152005.4 & +201605.8 & $1.5740(10)$ & $551.8(2)$ & 448 & 896 & CSS & $3,5,6$ & \\
\hline COINS J1546+0026 & 154609.5 & +002624.6 & 0.55 & 916.4 & 1782 & 1777 & GPS,CSO & 4 & 2 \\
\hline B2 $1600+33$ & 160207.3 & +332653.1 & 1.1 & 676.4 & 862 & $\ldots$ & GPS,CSS & $1,3,5,6$ & \\
\hline PKS $1622-253$ & 162546.9 & -252738.3 & 0.786 & 794.3 & 277 & 291 & FSRS & 2 & \\
\hline PKS 1622-29 & 162606.0 & -295127.0 & 0.815 & 781.6 & 266 & 298 & FSRS & 11 & \\
\hline 3C343 & 163433.8 & +624535.8 & 0.988 & 714.5 & 1194 & 1197 & CSS & $3,5,6,7$ & \\
\hline $1637+574$ & 163813.5 & +572024.0 & $0.7506(10)$ & $811.4(5)$ & 265 & 214 & FSRS & 2,8 & \\
\hline 3C343.1 & 163828.2 & +623444.3 & 0.75 & 811.7 & 1800 & 1787 & CSS & $3,5,6,7$ & \\
\hline $1642+690$ & 164407.9 & +685639.8 & 0.751 & 810.2 & 290 & 261 & FSRS & 2,8 & 2 \\
\hline $1656+053$ & 165833.5 & +051516.4 & 0.879 & 754.9 & 282 & 261 & FSRS & 2,8 & \\
\hline $1730-130$ & 173302.7 & -130449.5 & 0.902 & 745.8 & 284 & 280 & FSRS & 2 & \\
\hline $1741-038$ & 174358.9 & -035004.6 & 1.054 & 691.5 & 271 & 298 & FSRS & 2 & \\
\hline $1749+701$ & 174832.8 & +700550.8 & 0.770 & 801.5 & 278 & 278 & FSRS & 2,8 & \\
\hline $1803+784$ & 180045.7 & +782804.0 & 0.680 & 844.5 & 298 & $\ldots$ & FSRS & 2,8 & \\
\hline $1800+440$ & 180132.3 & +440421.9 & 0.663 & 845.1 & 288 & $\ldots$ & FSRS & 2,8 & \\
\hline COINS J1815+6127 & 181536.8 & +612711.6 & $0.601(3)$ & $887.2(1.7)$ & 1436 & 2090 & GPS,CSO & 4 & 2 \\
\hline $1823+568$ & 182407.1 & +565101.5 & $0.6640(10)$ & $853.6(5)$ & 288 & 260 & FSRS & 2 & \\
\hline $3 \mathrm{C} 380$ & 182931.8 & +484416.2 & 0.69 & 840.5 & 597 & 384 & CSS & 5,6 & 2 \\
\hline $4 C+29.56$ & $1831 \quad 14.9$ & +290710.3 & $0.842(3)$ & $771.1(1.3)$ & 1293 & 1493 & CSS & $3,5,6$ & 2 \\
\hline WMAP $1849+6705$ & 184916.1 & +670541.7 & $0.6570(10)$ & $857.2(5)$ & 283 & 296 & FSRS & 2,8 & \\
\hline TXS $1848+283$ & 185027.6 & +282513.2 & 2.56 & 398.99 & 1791 & 3583 & GPS & 1 & \\
\hline TXS $1851+488$ & 185228.5 & +485547.5 & $1.250(3)$ & $631.3(8)$ & 597 & $\ldots$ & GPS & 1 & \\
\hline $1921-293$ & 192451.1 & -291430.1 & $0.35263(18)$ & $1050.11(14)$ & 381 & $\ldots$ & FSRS & 2 & \\
\hline PKS 2000-330 & 200324.1 & -325145.1 & $3.7730(10)$ & $297.59(6)$ & 1720 & 1744 & GPS & 1 & \\
\hline $2007+777$ & 200530.9 & +775243.1 & 0.342 & 1058.4 & 394 & $\ldots$ & FSRS & 2,8 & \\
\hline PKS 2008-068 & 201112.2 & -064403.6 & $0.5470(10)$ & $918.2(6)$ & 1740 & 1791 & GPS & $1,3,7$ & \\
\hline COINS J2022+6136 & 202209.7 & +613658.8 & 0.227 & 1157 & 258 & 285 & GPS & 12 & 2 \\
\hline $2059+034$ & 210138.8 & +034131.3 & 1.013 & 705.6 & 242 & 290 & FSRS & 2 & \\
\hline PKS 2121-01 & 212339.1 & -011234.7 & $1.158(2)$ & $658.2(6)$ & 299 & & GPS,CSO & 1,10 & \\
\hline PKS 2126-15 & 212912.2 & -153841.0 & $3.2680(10)$ & $332.80(8)$ & 3583 & 3583 & GPS & 1,3 & \\
\hline PKS $2127+04$ & 213032.9 & +050217.5 & 0.99 & 713.8 & 893 & 896 & GPS & 1,3 & \\
\hline $2128-123$ & 213135.3 & -120704.8 & 0.501 & 945.3 & 384 & 366 & FSRS & 2 & \\
\hline PKS $2134+004$ & 213638.6 & +004154.2 & $1.9320(10)$ & $484.45(17)$ & 1791 & $\ldots$ & GPS & 3 & \\
\hline PKS 2135-209 & 213750.0 & -204231.7 & $0.63634(3)$ & $868.04(2)$ & 200 & 1682 & CSS & 6 & 2 \\
\hline $2145+067$ & 214805.5 & +065738.6 & 0.990 & 712.8 & 282 & $\ldots$ & FSRS & 2,8 & \\
\hline PKS $2149+056$ & 215137.9 & +055213.0 & $0.740(10)$ & $816.3(5)$ & 1790 & $\ldots$ & GPS & 1 & 1 \\
\hline $2155-152$ & 215806.8 & -150109.3 & 0.672 & 848.5 & 278 & 386 & FSRS & 2 & \\
\hline $2201+315$ & 220315.0 & +314538.3 & $0.2950(5)$ & $1096.8(4)$ & 398 & 280 & FSRS & 2,8 & \\
\hline $2216-038$ & 221852.0 & -033536.9 & 0.901 & 746.2 & 281 & 255 & FSRS & 2 & \\
\hline PKS $2230+11$ & 223236.4 & +114350.9 & 1.037 & 697.3 & 1752 & 1791 & CSS & 5,6 & \\
\hline $2234+282$ & 223622.5 & +282857.4 & 0.795 & 790.3 & 288 & 362 & FSRS & 2,8 & \\
\hline $3 \mathrm{C} 454.1$ & 225032.9 & +712919.2 & 1.841 & 499.97 & 896 & $\ldots$ & CSS & $3,5,6$ & \\
\hline $3 \mathrm{C} 454$ & 225134.7 & +184840.1 & 1.757 & 515.2 & 1316 & 1752 & CSS & $3,5,6$ & \\
\hline $3 \mathrm{C} 454.3$ & 225357.8 & +160853.6 & 0.859 & 763.1 & 252 & 266 & FSRS & 2,8 & \\
\hline $3 \mathrm{C} 455$ & 225503.9 & +131334.0 & 0.543 & 920.6 & 1790 & 1791 & CSS & $3,5,6$ & 2 \\
\hline $2255-282$ & 225806.0 & -275821.3 & $0.92584(15)$ & $737.69(6)$ & 273 & 296 & FSRS & 2 & \\
\hline 3C459 & 231635.2 & +040518.1 & $0.22012(3)$ & $1164.15(3)$ & 292 & 286 & CSS & 13 & \\
\hline HB89 2342+821 & 234403.8 & +822640.4 & 0.735 & 818.7 & 1791 & 1752 & GPS,CSS & $1,3,5,6$ & 2 \\
\hline HB89 $2344+092$ & 234636.8 & +093045.5 & 0.677 & 847.0 & 298 & 263 & FSRS & 2,8 & \\
\hline
\end{tabular}

Note. - Columns list the source name, Right Ascension and Declination, optical redshift, expected frequency of the corresponding HI $21 \mathrm{~cm}$ line, total integration times for each reduced spectra, radio source classification (GPS, CSS, CSO, FSRS), references for the classification, and references for any previous HI $21 \mathrm{~cm}$ observations. Numbers in parentheses indicate uncertainties in the final digit(s) of listed quantities, when available.

Classification references: 1 - de Vries, Barthel, \& O'Dea (1997); 2 - Healey et al. (2007); 3 - O'Dea \& Baum (1997); $4-$ Peck \& Taylor (2000); 5 - Fanti et al. (1990); 6 - Spencer et al. (1989); 7 - Morganti et al. (1997); 8-Augusto et al. (2006); 9 - Condon et al. (1977); 10 - Xiang et al. (2005); 11 - Jackson et al. (2002); 12 - Fomalont et al. (2000); 13 -Gelderman \& Whittle (1994).

Previous spectroscopy of the Hl $21 \mathrm{~cm}$ line references: 1 - Carilli et al. (1998); 2 - Vermeulen et al. (2003); 3 - Gupta et al. (2006); 4 Ishwara-Chandra et al. (2003); 5 - Curran et al. 2013.

Table 2

HI $21 \mathrm{~cm}$ Line Observations, Column Densities, and Source Sizes

\begin{tabular}{|c|c|c|c|c|c|c|c|c|c|c|}
\hline Source & $z$ & $\begin{array}{c}\nu \\
(\mathrm{MHz})\end{array}$ & $\begin{array}{c}\Delta v \\
\left(\mathrm{~km} \mathrm{~s}^{-1}\right)\end{array}$ & $\begin{array}{l}\mathrm{rms} \\
(\mathrm{mJy})\end{array}$ & $\begin{array}{c}\text { Ctnm } \\
(\mathrm{Jy})\end{array}$ & $\underset{\left(10^{20}\right.}{N_{\mathrm{HI}}}$ & $\begin{array}{l}\text { Prev. } N_{\mathrm{HI}} \\
\left.\mathrm{cm}^{-2}\right)\end{array}$ & $(\operatorname{arcsec})^{\mathrm{S}}$ & $(\mathrm{kpc})$ & Ref. \\
\hline $0018+729$ & $0.821(2)$ & $780.0(9)$ & $-5077,6080$ & 3.37 & $1.0033(6)$ & $<0.51$ & $\ldots$ & 0.018 & 0.14 & 1 \\
\hline LBQS $0106+0119$ & $2.009(5)$ & $472.1(8)$ & - & RFI & & & & 0.0106 & 0.09 & 2 \\
\hline COINS J0111+3906 & $0.66847(3)$ & $853.322(15)$ & $-1343,3015$ & 2.47 & $0.153(2)$ & $74(13)$ & $81(17)^{1}$ & 0.005 & 0.04 & 3 \\
\hline UM321 & $1.07481(15)$ & $684.60(5)$ & $-3813,26560$ & 8.3 & $1.306(18)$ & $<1.1$ & $\ldots$ & 0.0048 & 0.039 & 4 \\
\hline $3 \mathrm{C} 43$ & 1.459 & 577.6 & $-3047,2470$ & 12.8 & $5.97(4)$ & $<0.29$ & $\cdots$ & 2.60 & 22.2 & 5 \\
\hline $0133+476$ & 0.859 & 764.1 & $\cdots$ & RFI & & $\cdots$ & $\cdots$ & 0.0029 & 0.022 & 2 \\
\hline $3 \mathrm{C} 48$ & 0.367 & 1038 & $-432,568$ & 4.9 & $20.04(4)$ & $<0.037$ & . & 0.50 & 2.5 & 5 \\
\hline $3 \mathrm{C} 49$ & 0.62 & 876.8 & $\cdots$ & RFI & $\cdots$ & $\cdots$ & $1.1^{2}$ & 0.99 & 6.7 & 3 \\
\hline
\end{tabular}


Grasha et al.

Table 2 - Continued

\begin{tabular}{|c|c|c|c|c|c|c|c|c|c|c|}
\hline Source & $z$ & $\begin{array}{c}\nu \\
(\mathrm{MHz})\end{array}$ & $\begin{array}{c}\Delta v \\
\left(\mathrm{~km} \mathrm{~s}^{-1}\right)\end{array}$ & $\begin{array}{c}\mathrm{rms} \\
(\mathrm{mJy})\end{array}$ & $\begin{array}{c}\text { Ctnm } \\
\text { (Jy) }\end{array}$ & $\begin{array}{l}N_{\mathrm{HI}} \\
\quad\left(10^{20}\right.\end{array}$ & $\begin{array}{l}\text { Prev. } N_{\mathrm{HI}} \\
\left.\mathrm{cm}^{-2}\right)\end{array}$ & $\begin{array}{r}\mathrm{Si} \\
(\operatorname{arcsec})\end{array}$ & $(\mathrm{kpc})$ & Ref. \\
\hline $0138-097$ & 0.733 & 818.6 & $-2423,2711$ & 9.9 & $0.76(12)$ & $<1.9$ & $\ldots$ & 0.0019 & 0.014 & 4 \\
\hline PKS 0201+113 & $3.6390(10)$ & $306.19(7)$ & $-2976,10760$ & 7.13 & $0.331(8)$ & $<2.9$ & $\ldots$ & 0.002 & 0.015 & 1 \\
\hline $0212+735$ & 2.367 & 421.9 & $\ldots$ & RFI & $\ldots$ & $\ldots$ & $\ldots$ & 0.0131 & 0.108 & 2 \\
\hline B2 $0218+357$ & $0.944(2)$ & $730.7(8)$ & $-1559,4882$ & 6.1 & $1.559(4)$ & $<0.64$ & $\ldots$ & 0.0052 & 0.037 & 6 \\
\hline $3 \mathrm{C} 066 \mathrm{~A}$ & 0.444 & 982.7 & $-3179,2840$ & 8.2 & $2.83(16)$ & $<0.47$ & $\ldots$ & 0.0053 & 0.030 & 2 \\
\hline $0221+067$ & 0.511 & 940.0 & $-462,806$ & 5.9 & $0.961(10)$ & $<1.0$ & $\cdots$ & 0.0035 & 0.022 & 4 \\
\hline $4 C+34.07$ & $2.910(2)$ & $363.28(19)$ & & RFI & & $\ldots$ & $\ldots$ & 1.1 & 8.7 & 5 \\
\hline HB89 $0235+164$ & 0.940 & 732.2 & $-458,1628$ & 8.6 & $1.1(2)$ & $<1.3$ & $\ldots$ & 0.0021 & 0.017 & 2 \\
\hline PKS 0237-23 & 2.223 & 440.71 & $-327,1721$ & 4.10 & $3.42(2)$ & $<0.21$ & .. & 0.018 & 0.15 & 5 \\
\hline PKS $0239+108$ & 2.694 & 384.5 & $\ldots$ & RFI & $\ldots$ & $\ldots$ & $\mathrm{RFI}^{8}$ & 0.0024 & 0.019 & 4 \\
\hline B3 $0248+430$ & 1.31 & 614.9 & & RFI & & $\ldots$ & $<0.78^{3}$ & 0.06 & 0.5 & 5 \\
\hline $0306+102$ & 0.863 & 761.4 & $-12690,1262$ & 8.0 & $0.47960(2)$ & $<2.6$ & $\ldots$ & 0.0073 & 0.056 & 7 \\
\hline NGC 1275 & $0.01765(4)$ & $1395.77(5)$ & $-2242,2530$ & 4.8 & $23(3)$ & $<0.035$ & $\ldots$ & 0.102 & 0.00361 & 2 \\
\hline $\mathrm{OE}+131$ & 2.662 & 387.88 & $-8579,10542$ & 8.33 & $2.689(9)$ & $<0.63$ & $\mathrm{RFI}^{8}$ & 0.02 & 0.16 & 5 \\
\hline PKS 0327-241 & 0.895 & 748.6 & $-1668,979$ & 8.3 & $0.9197(4)$ & $<1.5$ & $\ldots$ & - & & $\ldots$ \\
\hline $0336-019$ & 0.852 & 767.0 & $-3379,3009$ & 6.5 & $2.26(8)$ & $<0.45$ & $\ldots$ & 0.0013 & 0.010 & 2 \\
\hline $0346-279$ & 0.991 & 712.4 & $-1366,418$ & 9.7 & $1.03(6)$ & $<1.6$ & $\ldots$ & 0.0095 & 0.076 & 4 \\
\hline $4 \mathrm{C}+76.03$ & 0.5985 & 888.6 & $-391,631$ & 6.75 & $6.618(7)$ & $3.7(1.1)$ & $2.5^{2}$ & 0.14 & 0.93 & 3 \\
\hline $0420-014$ & $0.91609(18)$ & $741.30(7)$ & $\ldots$ & RFI & $\ldots$ & $\ldots$ & $\ldots$ & 0.0017 & 0.013 & 2 \\
\hline $0422+004$ & 0.310 & 1084.3 & $\ldots$ & RFI & $\ldots$ & $\ldots$ & $\ldots$ & 0.0029 & 0.013 & 4 \\
\hline 3C119 & 1.023 & 702.1 & $-1221,809$ & 12.5 & $14.0(1.6)$ & $<0.15$ & $\ldots$ & 0.08 & 0.65 & 5 \\
\hline $0440-003$ & 0.844 & 769.3 & $-2391,1888$ & 8.1 & $2.37(4)$ & $<0.53$ & $\ldots$ & 0.0078 & 0.060 & 4 \\
\hline PKS 0454-234 & 1.003 & 708.1 & $\ldots$ & RFI & & $\ldots$ & $\ldots$ & 0.0024 & 0.019 & 2 \\
\hline PKS $0457+024$ & 2.384 & 419.7 & $-3714,6678$ & 5.23 & $0.390(19)$ & $<2.6$ & $\cdots$ & 0.0067 & 0.055 & 8 \\
\hline $0458-020$ & 2.286 & 432.3 & & RFI & & & $\ldots$ & 0.0046 & 0.038 & 2 \\
\hline PKS 0500+019 & $0.58457(2)$ & $896.398(11)$ & $-1191,314$ & 7.13 & $1.57(7)$ & $7.1(1.3)$ & $7.0(1.3)^{1}$ & 0.011 & 0.073 & 3 \\
\hline $3 \mathrm{C} 138$ & 0.76 & 807.1 & $-2775,2039$ & 7.76 & $11.42(4)$ & $<0.10$ & $<0.10^{2}$ & 0.68 & 5.0 & 3 \\
\hline PKS 0528-250 & 2.813 & 372.5 & $-5883,2197$ & 5.95 & $1.6(3.6)$ & $<0.81$ & $\ldots$ & 0.010 & 0.080 & 4 \\
\hline PKS 0539-057 & $0.839(3)$ & $772.4(1.3)$ & $-1497,2526$ & 8.7 & $0.91(9)$ & $<1.5$ & $\ldots$ & 0.0030 & 0.023 & 4 \\
\hline $3 \mathrm{C} 147$ & 0.55 & 916.4 & $-2522,717$ & 12 & $29.4(6)$ & $<0.070$ & $<0.085^{2}$ & 0.94 & 6.0 & 3 \\
\hline B3 $0552+398$ & $2.365(5)$ & $422.1(6)$ & $-5078,6761$ & 4.83 & $0.490(7)$ & $<1.9$ & $\ldots$ & 0.003 & 0.03 & 8 \\
\hline $0605-085$ & 0.872 & 757.8 & $-2297,1170$ & 7.5 & $2.820(14)$ & $<0.42$ & $\cdots$ & 0.0040 & 0.031 & 4 \\
\hline $0607-157$ & $0.3240(10)$ & $1072.8(8)$ & $\ldots$ & RFI & $\ldots$ & $\ldots$ & $\ldots$ & 0.0146 & 0.068 & 4 \\
\hline HB89 $0636+680$ & $3.1800(10)$ & $339.81(8)$ & $-10896,11619$ & 7.24 & $0.119(2)$ & $<7.9$ & $\cdots$ & 0.006 & 0.05 & 6 \\
\hline B2 $0711+35$ & 1.62 & 542.1 & $-375,4993$ & 8.52 & $0.87(2)$ & $<1.5$ & $\ldots$ & 0.006 & 0.05 & 8 \\
\hline $0735+178$ & 0.424 & 996.5 & $-1511,1569$ & 4.6 & $2.16(4)$ & $<0.34$ & $\ldots$ & 2.6 & 14 & 9 \\
\hline $0736+017$ & $0.189410(9)$ & $1194.21(2)$ & $-1854,2413$ & 5.8 & $2.54(3)$ & $<0.38$ & $\ldots$ & 0.0036 & 0.011 & 4 \\
\hline B2 $0738+31$ & $0.6314(16)$ & $870.7(9)$ & $-840,2672$ & 19.2 & $1.491(6)$ & $<2.4$ & $<0.45^{2}$ & 0.006 & 0.04 & 3 \\
\hline PKS $0742+10$ & $2.624(3)$ & $391.9(3)$ & $-5033,3071$ & 5.24 & $1.562(6)$ & $<0.58$ & $<1.4^{5}$ & 0.010 & 0.08 & 5 \\
\hline PKS 0743-006 & 0.994 & 712.3 & $\cdots$ & RFI & $\ldots$ & $\ldots$ & $\ldots$ & 0.005 & 0.04 & 5 \\
\hline $3 \mathrm{C} 186$ & $1.0670(19)$ & $687.2(6)$ & $-1719,2208$ & 4.34 & $2.99(2)$ & $<0.25$ & $\ldots$ & 2.2 & 18 & 5 \\
\hline PKS $0745+241$ & $0.4092(10)$ & $1008.0(7)$ & $-1890,2257$ & 4.8 & $1.038(9)$ & $<0.73$ & $\ldots$ & 0.0032 & 0.017 & 4 \\
\hline $0748+126$ & 0.889 & 750.9 & & RFI & & - & $\ldots$ & 0.0018 & 0.014 & 4 \\
\hline COINS J0753+4231 & $3.5892(8)$ & $309.51(5)$ & $-5318,13086$ & 8.32 & $0.7667(5)$ & $<1.4$ & $\cdots$ & 0.0086 & 0.06 & 6 \\
\hline HB89 $0754+100$ & $0.2660(10)$ & $1122.0(9)$ & $-491,939$ & 4.3 & $1.1(7)$ & $<0.73$ & $<0.83^{9}$ & 3.7 & 15 & 10 \\
\hline $3 \mathrm{C} 190$ & $1.1944(12)$ & $647.3(4)$ & $\ldots$ & RFI & $\ldots$ & $\ldots$ & $40^{4}$ & 4.1 & 34 & 5 \\
\hline SBS $0804+499$ & $1.430(2)$ & $584.5(5)$ & $\ldots$ & RFI & $\ldots$ & $\ldots$ & $<5.1^{10}$ & 0.0012 & 0.010 & 2 \\
\hline PKS $0823+033$ & 0.506 & 942.2 & $-1880,1825$ & 5.7 & $1.3(6)$ & $<0.76$ & $\cdots$ & 0.0038 & 0.023 & 4 \\
\hline PKS $0823-223$ & 0.910 & 742.7 & $-2907,1540$ & 8.6 & $0.64(6)$ & $<2.2$ & $\ldots$ & 0.0025 & 0.020 & 11 \\
\hline $0828+493$ & 0.548 & 916.6 & $-1150,803$ & 7.0 & $1.01(14)$ & $<1.2$ & $\ldots$ & 0.0064 & 0.041 & 6 \\
\hline $0836+710$ & $2.1720(10)$ & $447.80(14)$ & ... & RFI & $\ldots$ & $\ldots$ & $\ldots$ & 0.0087 & 0.075 & 2 \\
\hline $\mathrm{OJ}+287$ & 0.306 & 1087.6 & $\cdots$ & RFI & $\ldots$ & $\ldots$ & $\ldots$ & 0.0030 & 0.014 & 2 \\
\hline PKS 0858-279 & 2.152 & 450.6 & $-1924,3340$ & 13.9 & $0.86(1)$ & $<2.9$ & $\ldots$ & $\ldots$ & $\ldots$ & $\ldots$ \\
\hline TXS $0902+490$ & $2.6887(11)$ & $385.07(11)$ & $-3636,3916$ & 6.94 & $0.954(4)$ & $<1.5$ & $<7.2^{8}$ & 0.013 & 0.11 & 1 \\
\hline $0906+015$ & $1.020(3)$ & $701.8(1.1)$ & & RFI & $\ldots$ & $\ldots$ & .. & 0.0076 & 0.062 & 3 \\
\hline $3 \mathrm{C} 216$ & $0.6703(12)$ & $850.4(6)$ & $-353,3589$ & 8.06 & $6.41(4)$ & $1.8(4)$ & $1.2^{2}$ & 9.2 & 64.5 & 3 \\
\hline $0917+449$ & $2.1899(2)$ & $445.28(3)$ & & RFI & & $\ldots$ & $\ldots$ & 0.0028 & 0.024 & 2 \\
\hline $0919-260$ & 2.300 & 429.4 & $-4715,737$ & 4.2 & $0.86(11)$ & $<0.90$ & $\ldots$ & 0.0012 & 0.010 & 4 \\
\hline $0923+392$ & $0.6952(3)$ & $837.90(15)$ & $-1669,879$ & 8.6 & $3.1(2)$ & $<0.39$ & $\ldots$ & 0.0020 & 0.014 & 2 \\
\hline $0925-203$ & $0.34741(15)$ & $1053.71(2)$ & $-1790,1656$ & 7.9 & $0.89(9)$ & $<1.3$ & $\ldots$ & & & \\
\hline HB89 0954+556 & $0.896(8)$ & $749(3)$ & $-1837,862$ & 8.6 & $4.345(17)$ & $<0.32$ & $\ldots$ & 0.0089 & 0.071 & 4 \\
\hline HB89 $0954+658$ & 0.368 & 1037 & $-6182,1058$ & 6.8 & $0.7635(2)$ & $<1.4$ & $<11^{6}$ & 0.0023 & 0.012 & 4 \\
\hline $1004+141$ & $2.707(5)$ & $383.2(5)$ & $-4512,266$ & 9.4 & $1.305(15)$ & $<1.5$ & $\mathrm{RFI}^{8}$ & 0.0074 & 0.061 & 13 \\
\hline $3 \mathrm{C} 237$ & 0.87 & 759.6 & $-6394,4732$ & 6.29 & $9.47(6)$ & $<0.10$ & 20 & 1.3 & 10.1 & 5 \\
\hline PKS 1034-293 & 0.312 & 1082 & $-1129,1975$ & 4.4 & $1.06(11)$ & $<0.62$ & $\cdots$ & 0.013 & 0.006 & 2 \\
\hline $1055+018$ & $0.888(5)$ & $752(2)$ & $-3869,2270$ & 13 & $3.759(13)$ & $<0.54$ & $\ldots$ & 0.0032 & 0.025 & 4 \\
\hline $1116+128$ & $2.129(2)$ & $454.0(3)$ & $\ldots$ & RFI & $\ldots$ & $\ldots$ & $\ldots$ & 0.0094 & 0.081 & 2 \\
\hline PKS 1124-186 & 1.050 & 691.9 & $-2521,1682$ & 6.8 & $0.47(5)$ & $<2.6$ & $\ldots$ & 0.0004 & 0.003 & 4 \\
\hline B3 $1144+402$ & $1.0880(10)$ & $680.3(3)$ & $-1316,2171$ & 9.4 & $0.6038(4)$ & $<2.8$ & $\ldots$ & 0.0059 & 0.049 & 7 \\
\hline FBQS J1159+2914 & $0.7245(11)$ & $823.7(5)$ & $-7388,8262$ & 5.1 & $2.25(3)$ & $<0.33$ & $<1.8^{2}$ & 0.036 & 0.026 & 2 \\
\hline $1202-262$ & 0.789 & 794.0 & $-640,1326$ & 5.3 & $2.2090(10)$ & $<0.36$ & $\ldots$ & 0.003 & 0.03 & 4 \\
\hline B2 $1219+28$ & 0.102 & 1288 & $-807,1637$ & 6.8 & $0.9(1.7)$ & $<1.2$ & $\cdots$ & 0.0072 & 0.013 & 7 \\
\hline $1222+037$ & $0.9556(11)$ & $726.3(4)$ & $-3436,2880$ & 8.5 & $0.85(9)$ & $<1.7$ & $\ldots$ & 0.0015 & 0.012 & 4 \\
\hline
\end{tabular}


Table 2 - Continued

\begin{tabular}{|c|c|c|c|c|c|c|c|c|c|c|}
\hline Source & $z$ & $\begin{array}{c}\nu \\
(\mathrm{MHz})\end{array}$ & $\begin{array}{c}\Delta v \\
\left(\mathrm{~km} \mathrm{~s}^{-1}\right)\end{array}$ & $\begin{array}{c}\mathrm{rms} \\
(\mathrm{mJy})\end{array}$ & $\begin{array}{c}\text { Ctnm } \\
(\mathrm{Jy})\end{array}$ & $\begin{array}{l}N_{\mathrm{HI}} \\
\quad\left(10^{20}\right.\end{array}$ & $\begin{array}{l}\text { Prev. } N_{\mathrm{HI}} \\
\left.\mathrm{cm}^{-2}\right)\end{array}$ & $\begin{array}{r}\mathrm{S} \\
(\operatorname{arcsec})\end{array}$ & (kpc) & Ref. \\
\hline PG $1222+216$ & $0.4320(10)$ & $991.9(7)$ & $-1988,2095$ & 8.2 & $2.06(9)$ & $<0.64$ & $\cdots$ & 0.0034 & 0.019 & 2 \\
\hline B2 $1225+36$ & $1.973(2)$ & $477.8(3)$ & $\cdots$ & RFI & $\cdots$ & $\cdots$ & $\cdots$ & 0.060 & 0.51 & 5 \\
\hline $3 \mathrm{C} 273$ & $0.15834(7)$ & $1226.24(7)$ & $-9459,2793$ & 4.2 & $46.69(7)$ & $<0.014$ & $\cdots$ & 0.261 & 0.0706 & 2 \\
\hline COINS J1244+4048 & $0.813(2)$ & $783.5(9)$ & $-2334,2138$ & 7.5 & $1.62(3)$ & $<0.70$ & $\cdots$ & 0.04 & 0.3 & 12 \\
\hline $3 \mathrm{C} 279$ & $0.5362(4)$ & $924.6(2)$ & $-1768,3010$ & 4.4 & $11.1(2)$ & $<0.068$ & $\cdots$ & 0.0032 & 0.020 & 2 \\
\hline $1308+326$ & 0.996 & 711.6 & $\ldots$ & RFI & $\ldots$ & $\ldots$ & $\cdots$ & 0.0038 & 0.031 & 2 \\
\hline $3 \mathrm{C} 287$ & 1.055 & 691.2 & $-4133,4190$ & 4.03 & $10.5(3)$ & $<0.066$ & $\cdots$ & 0.048 & 0.39 & 5 \\
\hline $3 \mathrm{C} 286$ & $0.8494(11)$ & $768.0(5)$ & $-3306,3466$ & 6.50 & $19.39(9)$ & $<0.052$ & $\ldots$ & 3.2 & 25 & 5 \\
\hline HB89 $1333+459$ & $2.4490(10)$ & $411.83(12)$ & $-8575,6741$ & 4.53 & $0.102(2)$ & $<9.2$ & $\cdots$ & 1.8 & 15 & 6 \\
\hline $1354+195$ & 0.720 & 824.8 & $-3694,2424$ & 8.2 & $3.13(7)$ & $<0.38$ & $\cdots$ & 0.0123 & 0.089 & 2 \\
\hline B3 $1355+441$ & 0.646 & 862.9 & $\ldots$ & RFI & $\ldots$ & $\ldots$ & $33^{2}$ & 0.017 & 0.12 & 3 \\
\hline $1418+546$ & $0.1526(3)$ & $1232.4(3)$ & $-3353,4972$ & 6.5 & $0.91(7)$ & $<1.2$ & $<2.7^{6}$ & 0.0038 & 0.010 & 2 \\
\hline 3C305.1 & 1.132 & 666.2 & $\ldots$ & RFI & $\ldots$ & $\ldots$ & $\ldots$ & 2.34 & 19 & 5 \\
\hline 3C309.1 & 0.905 & 745.6 & $-2810,4021$ & 7.89 & $11.457(5)$ & $<0.11$ & $\cdots$ & 2.11 & 16.5 & 5 \\
\hline $1504-166$ & $0.876(4)$ & $757.2(1.6)$ & $-5553,3412$ & 9.7 & $2.8(2)$ & $<0.54$ & $\cdots$ & 0.0017 & 0.0013 & 2 \\
\hline $3 \mathrm{C} 318$ & $1.5740(10)$ & $551.8(2)$ & $-962,869$ & 5.89 & $6.3(2)$ & $<0.13$ & $\cdots$ & 1.05 & 8.98 & 5 \\
\hline COINS J1546+0026 & 0.55 & 916.4 & $-2895,2676$ & 2.99 & $2.027(11)$ & $<0.25$ & $<0.25^{2}$ & 0.010 & 0.06 & 3 \\
\hline B2 $1600+33$ & 1.1 & 676.4 & $\cdots$ & RFI & $\ldots$ & $\cdots$ & $\ldots$ & 0.06 & 0.5 & 5 \\
\hline PKS $1622-253$ & 0.786 & 794.3 & $-3555,1566$ & 9.0 & $2.44701(15)$ & $<0.55$ & $\cdots$ & 0.004 & 0.03 & 4 \\
\hline PKS 1622-29 & 0.815 & 781.6 & $-2354,1570$ & 9.3 & $2.830(9)$ & $<0.50$ & $\cdots$ & 0.0160 & 0.121 & 2 \\
\hline $3 \mathrm{C} 343$ & 0.988 & 714.5 & $-3491,2250$ & 5.68 & $8.56(7)$ & $<0.11$ & $\ldots$ & 0.20 & 1.6 & 5 \\
\hline $1637+574$ & $0.7506(10)$ & $811.4(5)$ & $-8992,3641$ & 6.2 & $0.9(3)$ & $<0.99$ & $\cdots$ & 5.3 & 39 & 9 \\
\hline 3C343.1 & 0.75 & 811.7 & $-3494,3306$ & 5.52 & $7.28(6)$ & $<0.11$ & $\ldots$ & 0.24 & 1.8 & 5 \\
\hline $1642+690$ & 0.751 & 810.2 & $-7376,2826$ & 8.2 & $1.87(5)$ & $<0.64$ & $<1.3^{2}$ & 0.0041 & 0.030 & 2 \\
\hline $1656+053$ & 0.879 & 754.9 & $-2892,2922$ & 9.4 & $1.93(5)$ & $<0.77$ & $\ldots$ & 2.4 & 19 & 9 \\
\hline $1730-130$ & 0.902 & 745.8 & $-7145,6534$ & 11 & $6.7(2)$ & $<0.27$ & $\ldots$ & 0.0045 & 0.035 & 2 \\
\hline $1741-038$ & 1.054 & 567.3 & $\ldots$ & RFI & $\ldots$ & $\ldots$ & $\cdots$ & 0.0016 & 0.013 & 2 \\
\hline $1749+701$ & 0.770 & 801.5 & $-5764,2998$ & 6.9 & $1.3(8)$ & $<0.78$ & $\ldots$ & 0.0047 & 0.035 & 2 \\
\hline $1803+784$ & 0.680 & 844.5 & $-2230,2474$ & 6.0 & $2.1(2)$ & $<0.41$ & $\cdots$ & 0.0016 & 0.011 & 2 \\
\hline $1800+440$ & 0.663 & 845.1 & $\ldots$ & RFI & $\ldots$ & $\ldots$ & $\cdots$ & 0.0009 & 0.006 & 2 \\
\hline COINS J1815+6127 & $0.601(3)$ & $887.2(1.7)$ & $-1693,2244$ & 2.64 & $0.53(6)$ & $7(3)$ & $4.4^{2}$ & 0.010 & 0.068 & 3 \\
\hline $1823+568$ & $0.6640(10)$ & $853.6(5)$ & $\ldots$ & RFI & $\ldots$ & $\cdots$ & $\cdots$ & 0.0061 & 0.043 & 2 \\
\hline $3 \mathrm{C} 380$ & 0.69 & 840.5 & $-3688,4958$ & 12.4 & $21.7(2)$ & $<0.081$ & $<0.19^{2}$ & 1.3 & 9.0 & 3 \\
\hline $4 C+29.56$ & $0.842(3)$ & $771.1(1.3)$ & $-1656,1998$ & 1.86 & $3.90(1)$ & $<0.19$ & $<1.6^{2}$ & 2.9 & 20 & 3 \\
\hline WMAP $1849+6705$ & $0.6570(10)$ & $857.2(5)$ & $\ldots$ & RFI & $\ldots$ & $\cdots$ & $\cdots$ & 0.0026 & 0.018 & 2 \\
\hline TXS $1848+283$ & 2.56 & 398.99 & $-445,6101$ & 6.55 & $0.181(6)$ & $<7.6$ & $\cdots$ & $\ldots$ & $\ldots$ & $\cdots$ \\
\hline TXS $1851+488$ & $1.250(3)$ & $631.3(8)$ & $\ldots$ & RFI & $\ldots$ & $\cdots$ & $\cdots$ & 0.005 & 0.04 & 6 \\
\hline $1921-293$ & $0.35263(18)$ & $1050.11(14)$ & $\cdots$ & RFI & $\cdots$ & $\cdots$ & $\cdots$ & 0.0067 & 0.033 & 2 \\
\hline PKS 2000-330 & $3.7730(10)$ & $297.59(6)$ & $-4352,3697$ & 10.2 & $0.16(3)$ & $<9.5$ & $\cdots$ & $\cdots$ & $\cdots$ & $\cdots$ \\
\hline $2007+777$ & 0.342 & 1058.4 & $-846,970$ & 4.2 & $1.01(10)$ & $<0.64$ & $<1.8^{6}$ & 0.0048 & 0.023 & 13 \\
\hline PKS 2008-068 & $0.5470(10)$ & $918.2(6)$ & $-1858,340$ & 3.70 & $2.135(9)$ & $<0.30$ & $\ldots$ & 0.30 & 0.19 & 5 \\
\hline COINS J2022+6136 & 0.227 & 1157 & $-9869,1621$ & 7.2 & $1.99(15)$ & $<0.61$ & $<0.36^{2}$ & 0.010 & 0.036 & 5 \\
\hline $2059+034$ & 1.013 & 705.6 & $\cdots$ & RFI & $\ldots$ & $\cdots$ & $\cdots$ & 0.0016 & 0.013 & 4 \\
\hline PKS 2121-01 & $1.158(2)$ & $658.2(6)$ & $-944,20$ & 49 & $1.548(7)$ & $<6.0$ & $<6.9^{7}$ & 0.088 & 0.73 & 14 \\
\hline PKS 2126-15 & $3.2680(10)$ & $332.80(8)$ & $-4982,4118$ & 5.68 & $0.101(9)$ & $<7.2$ & $\ldots$ & 0.008 & 0.06 & 5 \\
\hline PKS $2127+04$ & 0.99 & 713.8 & $-972,1365$ & 6.03 & $5.04(8)$ & $<0.20$ & $\cdots$ & 0.030 & 0.24 & 5 \\
\hline $2128-123$ & 0.501 & 945.3 & $-1527,9545$ & 6.3 & $1.84(3)$ & $<0.59$ & $\cdots$ & 0.0105 & 0.0633 & 4 \\
\hline PKS 2134+004 & $1.9320(10)$ & $484.45(17)$ & $\cdots$ & RFI & $\ldots$ & $\ldots$ & $\cdots$ & 0.002 & 0.017 & 5 \\
\hline $2145+067$ & 0.990 & 712.8 & $-1432,993$ & 14 & $3.355(2)$ & $<0.68$ & $\cdots$ & 0.0071 & 0.057 & 2 \\
\hline PKS $2149+056$ & $0.740(10)$ & $816.3(5)$ & $-3595,1873$ & 3.39 & $0.507(3)$ & $<0.98$ & $<5.8^{1}$ & 0.004 & 0.03 & 3 \\
\hline $2155-152$ & 0.672 & 848.5 & $-2189,1779$ & 8.3 & $2.68(16)$ & $<0.43$ & $\ldots$ & 0.0072 & 0.051 & 2 \\
\hline $2201+315$ & $0.2950(5)$ & $1096.8(4)$ & $-1521,1395$ & 9.5 & $1.9(2.2)$ & $<0.48$ & $\cdots$ & 0.0054 & 0.024 & 2 \\
\hline $2216-038$ & 0.901 & 746.2 & $-2745,1660$ & 9.9 & $1.372(2)$ & $<1.2$ & $\ldots$ & 0.0005 & 0.004 & 15 \\
\hline PKS $2230+11$ & 1.037 & 697.3 & $-3036,2899$ & 4.87 & $7.50(9)$ & $<0.11$ & $\cdots$ & 2.6 & 21 & 16 \\
\hline $2234+282$ & 0.795 & 790.3 & $-5071,4060$ & 8.2 & $0.597(5)$ & $<2.1$ & $\ldots$ & 0.0011 & 0.083 & 4 \\
\hline $3 \mathrm{C} 454.1$ & 1.841 & 499.97 & $\ldots$ & RFI & $\ldots$ & $\cdots$ & $\ldots$ & 1.6 & 14 & 5 \\
\hline $3 \mathrm{C} 454$ & 1.757 & 515.2 & $-1670,897$ & 7.49 & $4.87(3)$ & $<0.24$ & $\cdots$ & 0.66 & 5.6 & 5 \\
\hline $3 \mathrm{C} 454.3$ & 0.859 & 763.1 & $-8290,1838$ & 9.2 & $13.87(11)$ & $<0.10$ & $\ldots$ & 0.0078 & 0.060 & 2 \\
\hline 3C455 & 0.543 & 920.6 & $-892,3709$ & 2.88 & $4.45(5)$ & $1.8(6)$ & $0.42^{2}$ & 2.6 & 17 & 3 \\
\hline $2255-282$ & $0.92584(15)$ & $737.69(6)$ & $\ldots$ & RFI & $\ldots$ & $\ldots$ & $\ldots$ & 0.0050 & 0.039 & 4 \\
\hline $3 \mathrm{C} 459$ & $0.22012(3)$ & $1164.15(3)$ & $-9191,3515$ & 4.9 & $5.23(3)$ & $<0.16$ & $\ldots$ & 1.86 & 6.55 & 17 \\
\hline HB89 2342+821 & 0.735 & 818.7 & $-3211,3413$ & 5.14 & $4.53(4)$ & $<0.16$ & $<0.16^{2}$ & 0.267 & 1.94 & 3 \\
\hline HB89 2344+092 & 0.677 & 847.0 & $-5233,2063$ & 6.4 & $2.233(2)$ & $<0.40$ & $\ldots$ & 0.0092 & 0.065 & 4 \\
\hline
\end{tabular}


Table 2 - Continued

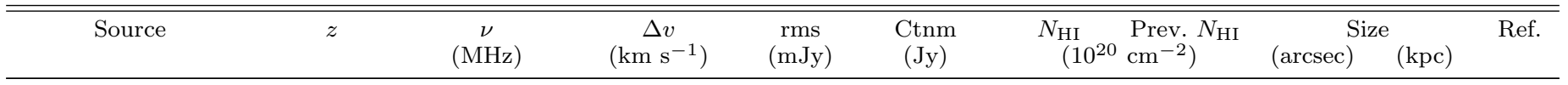

Note. - Columns list the source name, optical redshift, centroid or expected frequency for the HI 21 cm absorption line, velocity range searched (blue, red limits), spectral rms noise in the search region, the continuum flux density at the expected redshifted line frequency, the measured column density or $3 \sigma$ upper limit, previously measured column densities (or upper limits), largest projected angular size and linear size, respectively, and references for the continuum source characteristics. Numbers in parentheses show uncertainties in the final digit(s). Detections and non-detections assume $f=1$, and $T_{s}=100 \mathrm{~K}$. Non-detections also assume a line width of $30 \mathrm{~km} \mathrm{~s}{ }^{-1}$ (Sec. 4.3 .

References for previous HI $21 \mathrm{~cm}$ observations (non-detections are converted to our standard linewidth and spin temperature to aid in comparison): 1 - Carilli et al. (1998); 2 - Vermeulen et al. (2003); 3 - Gupta et al. (2006); 4 - Ishwara-Chandra et al. (2003); 5 - Curran et al. (2013); 6 Aditya \& Kanekar (2018b); 7 -Aditya \& Kanekar (2018a); 8-Curran et al. (2017); 9-Curran et al. (2019); 10 - Aditya et al. (2016). References for sizes: 1 - Stanghellini et al. (1990); 2 - Gurvits et al. (1999); 3 - Gupta et al. (2006); 4 - Fomalont et al. (2000); 5 - O'Dea \& Baum (1997); 6 - Xu et al. (1995); 7 - Fey \& Charlot (2000); 8- Menon (1983); 9 - Murphy et al. (1993); 10 - Antonucci \& Ulvestad (1985); 11 - Shen et al. (1998); 12 - Fanti et al. (2001); 13 - Fey \& Charlot (1997); 14-Xiang et al. (2005); 15 - Shen et al. (1997); $16-$ Fanti et al. (1990); 17 - Morganti, Killeen, \& Tadhunter (1993). 
Table 3

$\mathrm{OH} 18 \mathrm{~cm}$ Observations and Column Density Limits

\begin{tabular}{|c|c|c|c|c|c|c|c|c|c|c|c|c|}
\hline \multirow[b]{2}{*}{ Source } & \multirow[b]{2}{*}{$z$} & \multicolumn{3}{|c|}{$1612 \mathrm{MHz}$} & \multicolumn{3}{|c|}{$1667 \mathrm{MHz}$} & \multicolumn{3}{|c|}{$1720 \mathrm{MHz}$} & \multirow[b]{2}{*}{$\begin{array}{c}\text { Cntm } \\
(\mathrm{Jy})\end{array}$} & \multirow[b]{2}{*}{$\begin{array}{c}N_{\mathrm{OH}} / T_{x} \\
\left(10^{13} \mathrm{~cm}^{-2} \mathrm{~K}^{-1}\right)\end{array}$} \\
\hline & & $\begin{array}{c}\nu \\
(\mathrm{MHz})\end{array}$ & $\begin{array}{c}\Delta v \\
\left(\mathrm{~km} \mathrm{~s}^{-1}\right)\end{array}$ & $\begin{array}{c}\mathrm{rms} \\
(\mathrm{mJy})\end{array}$ & $\begin{array}{c}\nu \\
(\mathrm{MHz})\end{array}$ & $\begin{array}{c}\Delta v \\
\left(\mathrm{~km} \mathrm{~s}^{-1}\right)\end{array}$ & $\begin{array}{c}\mathrm{rms} \\
(\mathrm{mJy})\end{array}$ & $\begin{array}{c}\nu \\
(\mathrm{MHz})\end{array}$ & $\begin{array}{c}\Delta v \\
\left(\mathrm{~km} \mathrm{~s}^{-1}\right)\end{array}$ & $\begin{array}{c}\mathrm{rms} \\
(\mathrm{mJy})\end{array}$ & & \\
\hline $0018+729$ & 0.821 & 885.35 & & RFI & 915.63 & $-8945,9866$ & 2.41 & 944.83 & & & $0.9244(5)$ & $<6.0$ \\
\hline LBQS 0106+0119 & 2.009 & 535.80 & $-10741,7163$ & 5.5 & 554.12 & $-3179,2231$ & 5.5 & 571.79 & $-1681,3562$ & 5.1 & $2.7(2)$ & $<3.8$ \\
\hline UM321 & 1.07481 & 777.05 & $-4688,3492$ & 9.1 & 803.62 & $-4357,4894$ & 6.1 & 829.25 & & RFI & $1.355(18)$ & $<8.9$ \\
\hline $3 \mathrm{C} 43$ & 1.459 & 655.64 & $-153,1868$ & 12.3 & 678.06 & $-3188,491$ & 19.1 & 699.69 & $-1257,2514$ & 18.2 & $5.25(3)$ & $<8.5$ \\
\hline $0133+476$ & 0.859 & 867.26 & $\ldots$ & RFI & 896.91 & $-1834,572$ & 3.9 & 925.51 & $-1000,5,900$ & 6.1 & $1.983(11)$ & $<4.6$ \\
\hline $3 \mathrm{C} 48$ & 0.367 & 1179.4 & $-3967,3659$ & 8.1 & 1219.7 & $-3755,3618$ & 7.1 & 1258.6 & $-5093,623$ & 11.1 & $17.93(4)$ & $<0.85$ \\
\hline PKS $0201+113$ & 3.6390 & 347.54 & $-3762,7969$ & 9.78 & 359.42 & $-4905,3186$ & 10.1 & 370.88 & $-10440,5645$ & 11.9 & $0.365(8)$ & $<41$ \\
\hline $0212+735$ & 2.367 & 478.83 & . . & RFI & 495.21 & $\ldots$ & RFI & 511.00 & & RFI & $\ldots$ & 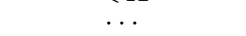 \\
\hline $3 \mathrm{C} 066 \mathrm{~A}$ & 0.444 & 1116.5 & $\ldots$ & RFI & 1154.7 & $-6314,1215$ & 8.1 & 1191.5 & $-5660,2894$ & 5.1 & $2.54(14)$ & $<7.2$ \\
\hline B2 $0218+357$ & 0.944 & 829.34 & $-4722,3917$ & 6.8 & 857.69 & ... & RFI & 885.05 & & RFI & $1.492(4)$ & $<78^{\mathrm{a}}$ \\
\hline $0221+067$ & 0.511 & 1067.0 & $\ldots$ & RFI & 1103.5 & $\ldots$ & RFI & 1138.7 & $\ldots$ & RFI & & $\ldots$ \\
\hline $4 \mathrm{C}+34.07$ & 2.91 & 412.34 & $-10626,7180$ & 12.2 & 426.43 & $\ldots$ & RFI & 440.03 & $\ldots$ & Nor & $3.61(9)^{\mathrm{a}}$ & $<77^{\mathrm{a}}$ \\
\hline HB89 0235+164 & 0.94 & 831.05 & $-5142,4598$ & 5.6 & 859.46 & $-3222,6719$ & 5.4 & 886.87 & $-11266,903$ & 5.6 & $1.0(2)$ & $<13$ \\
\hline PKS $0237-23$ & 2.223 & 500.23 & & RFI & 517.33 & ... & RFI & 533.83 & & & & \\
\hline PKS $0239+108$ & 2.694 & 436.45 & $-2064,2710$ & 18 & 451.37 & $\ldots$ & RFI & 465.76 & $\ldots$ & RFI & $1.41(14)^{\mathrm{a}}$ & $<281^{\mathrm{a}}$ \\
\hline B3 $0248+430$ & 1.31 & 697.94 & $-114,2034$ & 7.8 & 721.80 & $-1121,4444$ & 7.8 & 744.82 & & RFI & $0.782(3)$ & $<22$ \\
\hline $0306+102$ & 0.863 & 865.40 & $\ldots$ & RFI & 894.99 & $-9786,297$ & 4.1 & 923.53 & $-413,2378$ & 5.4 & $0.48210(2)$ & $<20$ \\
\hline $\mathrm{OE}+131$ & 2.662 & 440.26 & $-2956,2015$ & 13.9 & 455.31 & & RFI & 469.83 & & $\ldots$ & $2.586(8)^{\mathrm{a}}$ & $<112^{\mathrm{a}}$ \\
\hline PKS 0327-241 & 0.895 & 850.78 & $-3706,3729$ & 5.6 & 879.87 & & & 907.93 & . . & & $0.8660(4)^{\mathrm{a}}$ & $<108^{a}$ \\
\hline $0336-019$ & 0.852 & 870.54 & ... & RFI & 900.30 & $-7791,2032$ & 4.4 & 929.01 & . & RFI & $2.22(8)$ & $<46$ \\
\hline $0346-279$ & 0.991 & 809.76 & & RFI & 837.45 & $-3133,2595$ & 4.5 & 864.15 & $-4422,4,182$ & 4.1 & $0.98(6)$ & $<8.6$ \\
\hline $4 \mathrm{C}+76.03$ & 0.5985 & 1008.6 & $-751,3081$ & 6.32 & 1043.0 & $-1713,2109$ & 4.37 & 1076.3 & & RFI & $6.243(7)$ & $<1.4$ \\
\hline $0420-014$ & 0.914 & 841.42 & $-3048,2077$ & 5.9 & 870.91 & . . & RFI & 897.94 & $-7164,1240$ & 7.9 & $2.02(13)^{\mathrm{a}}$ & $<49^{\mathrm{a}}$ \\
\hline $0422+004$ & 0.31 & 1230.7 & $-5819,2609$ & 4.9 & 1272.8 & $-3087,1788$ & 5.8 & 1313.4 & $-3337,270$ & 3.4 & $0.51(5)$ & $<24$ \\
\hline 3C119 & 1.023 & 796.95 & $-4292,2897$ & 8.56 & 824.20 & $\ldots$ & RFI & 850.48 & $\ldots$ & $\ldots$ & $13.7(1.7)^{\mathrm{a}}$ & $<11^{\mathrm{a}}$ \\
\hline $0440-003$ & 0.844 & 874.31 & & RFI & 904.21 & $-6562,3285$ & 3.8 & 933.04 & $-3392,142$ & 5.7 & $2.60(4)$ & $<3.4$ \\
\hline PKS 0454-234 & 1.003 & 804.91 & $-10165,2834$ & 8.9 & 832.43 & $-4239,6170$ & 8.4 & 858.98 & $\ldots$ & RFI & $1.58(11)$ & $<10$ \\
\hline PKS $0457+024$ & 2.384 & 476.43 & 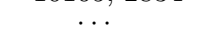 & RFI & 492.72 & & RFI & 508.43 & $\ldots$ & & & $\ldots$ \\
\hline $0458-020$ & 2.286 & 490.64 & $\ldots$ & RFI & 507.41 & $\cdots$ & RFI & 523.59 & .. & RFI & $\ldots$ & $\ldots$ \\
\hline $3 \mathrm{C} 138$ & 0.76 & 916.04 & $-1522,2154$ & 6.61 & 947.36 & $-3922,2256$ & 6.84 & 977.57 & $-471,2862$ & 7.91 & $10.5(4)$ & $<1.4$ \\
\hline PKS $0528-250$ & 2.813 & 422.82 & $-3173,3329$ & 2.84 & 437.28 & $-1442,12882$ & 11.8 & 451.23 & & $\ldots$ & $1.5(2.9)$ & $<19$ \\
\hline PKS $0539-057$ & 0.839 & 876.69 & $\ldots$ & RFI & 906.67 & $-6194,4,023$ & 4.5 & 935.58 & $-4909,3326$ & 6.7 & $0.91(9)$ & $<12$ \\
\hline $3 \mathrm{C} 147$ & 0.55 & 1040.2 & $-4914,2349$ & 9.1 & 1075.7 & $-1361,2652$ & 9.2 & 1110.0 & $-4208,788$ & 11.2 & $26.2(6)$ & $<0.68$ \\
\hline $0605-085$ & 0.872 & 861.23 & & RFI & 890.68 & $-7276,1846$ & 6.5 & 919.09 & $-6724,3551$ & 5.6 & $2.853(14)$ & $<5.4$ \\
\hline $0607-157$ & 0.324 & 1217.7 & $-3768,5588$ & 10 & 1259.3 & $-4729,270$ & 5.7 & 1299.5 & $-837,737$ & 10.2 & $2.30(3)$ & $<5.2$ \\
\hline HB89 $0636+680$ & 3.1800 & 385.70 & $-9194,13121$ & 4.73 & 398.89 & $-10496,9695$ & 7.69 & 411.61 & $-12972,6621$ & 7.99 & $0.128(2)$ & $<173$ \\
\hline B2 $0711+35$ & 1.62 & 615.36 & $\ldots$ & RFI & 636.40 & $\ldots$ & RFI & 656.69 & & $\ldots$ & $\ldots$ & $\cdots$ \\
\hline $0735+178$ & 0.424 & 1132.2 & $\ldots$ & RFI & 1170.9 & $-6427,3814$ & 3.8 & 1208.2 & $-4904,5021$ & 4.6 & $2.15(5)$ & $<4.0$ \\
\hline $0736+017$ & 0.191 & 1355.5 & $-4279,5908$ & 3.3 & 1401.8 & $-1935,7488$ & 3.6 & 1446.5 & $\ldots$ & RFI & $2.53(3)$ & $<3.2$ \\
\hline B2 $0738+31$ & 0.63139 & 988.26 & $-2886,869$ & 2.71 & 1022.1 & $-1865,3447$ & 2.22 & 1054.6 & .. & & $1.68(7)$ & $<2.7$ \\
\hline PKS $0742+10$ & 2.624 & 444.88 & $\cdots$ & RFI & 490.09 & $\ldots$ & RFI & 474.76 & $\cdots$ & RFI & $\ldots$ & $\cdots$ \\
\hline PKS 0743-006 & 0.994 & 808.54 & $-2083,2225$ & 4.79 & 836.19 & $-3203,3073$ & 5.6 & 862.85 & $-2149,981$ & 15.9 & $0.672(6)$ & $<16$ \\
\hline $3 \mathrm{C} 186$ & 1.067 & 779.99 & $-5121,5583$ & 2.89 & 806.66 & $-2110,1973$ & 6.71 & 832.38 & $-6641,2892$ & 3.01 & $2.465(19)$ & $<5.3$ \\
\hline PKS $0745+241$ & 0.4092 & 1144.1 & $-2679,2352$ & 4.5 & 1183.2 & & RFI & 1220.9 & & $\ldots$ & $1.007(10)^{\mathrm{a}}$ & $<92^{\mathrm{a}}$ \\
\hline $0748+126$ & 0.889 & 853.48 & & RFI & 882.67 & $\cdots$ & RFI & 910.82 & $-4208,762$ & 8.6 & $1.49(4)^{\mathrm{b}}$ & $<120^{\mathrm{b}}$ \\
\hline COINS J0753+4231 & 3.58925 & 351.31 & $-5317,13086$ & 6.39 & 363.32 & $-3452,6552$ & 6.59 & 374.90 & $-3395,1195$ & 6.81 & $0.7602(2)$ & $<13$ \\
\hline HB89 $0754+100$ & 0.266 & 1273.5 & $-3888,1997$ & 3.8 & 1317.0 & $-4774,4559$ & 3.7 & 1359.0 & $-3082,5301$ & 3.5 & $1.1(8)$ & $<6.7$ \\
\hline SBS $0804+499$ & 1.43 & 663.47 & & RFI & 686.16 & & RFI & 708.04 & & RFI & & \\
\hline PKS $0823+033$ & 0.506 & 1070.5 & $-2930,4071$ & 4.4 & 1107.1 & $-3752,3288$ & 4.6 & 1142.5 & $-506,3005$ & 4.2 & $1.3(6)$ & $<8.3$ \\
\hline PKS 0823-223 & 0.91 & 844.10 & $-2593,2451$ & 6.4 & 872.96 & & RFI & 900.80 & $-6656,1531$ & 5.8 & $0.61(6)^{\mathrm{a}}$ & $<177^{\mathrm{a}}$ \\
\hline $0828+493$ & 0.548 & 1041.5 & $-2737,2733$ & 5.1 & 1077.1 & $-2476,4761$ & 4.8 & 1111.5 & $-4733,1471$ & 3.6 & $0.92(14)$ & $<10$ \\
\hline $0836+710$ & 2.172 & 508.27 & $\ldots$ & RFI & 525.65 & $\ldots$ & RFI & 542.41 & & RFI & & 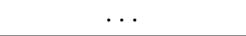 \\
\hline
\end{tabular}


Table 3 - Continued

\begin{tabular}{|c|c|c|c|c|c|c|c|c|c|c|c|c|}
\hline \multirow[b]{2}{*}{ Source } & \multirow[b]{2}{*}{$z$} & \multicolumn{3}{|c|}{$1612 \mathrm{MHz}$} & \multicolumn{3}{|c|}{$1667 \mathrm{MHz}$} & \multicolumn{3}{|c|}{$1720 \mathrm{MHz}$} & \multirow[b]{2}{*}{$\begin{array}{l}\text { Cntm } \\
(\mathrm{Jy})\end{array}$} & \multirow[b]{2}{*}{$\begin{array}{c}N_{\mathrm{OH}} / T_{x} \\
\left(10^{13} \mathrm{~cm}^{-2} \mathrm{~K}^{-1}\right)\end{array}$} \\
\hline & & $\begin{array}{c}\nu \\
(\mathrm{MHz})\end{array}$ & $\begin{array}{c}\Delta v \\
\left(\mathrm{~km} \mathrm{~s}^{-1}\right)\end{array}$ & $\begin{array}{l}\mathrm{rms} \\
(\mathrm{mJy})\end{array}$ & $\begin{array}{c}\nu \\
(\mathrm{MHz})\end{array}$ & $\begin{array}{c}\Delta v \\
\left(\mathrm{~km} \mathrm{~s}^{-1}\right)\end{array}$ & $\begin{array}{l}\mathrm{rms} \\
(\mathrm{mJy})\end{array}$ & $\begin{array}{c}\nu \\
(\mathrm{MHz})\end{array}$ & $\begin{array}{c}\Delta v \\
\left(\mathrm{~km} \mathrm{~s}^{-1}\right)\end{array}$ & $\begin{array}{l}\mathrm{rms} \\
(\mathrm{mJy})\end{array}$ & & \\
\hline TXS $0902+490$ & 2.6887 & 437.07 & $-6130,1099$ & 5.38 & 452.02 & & RFI & 466.43 & $\ldots$ & RFI & $0.907(3)^{\mathrm{a}}$ & $<124^{\mathrm{a}}$ \\
\hline $0906+015$ & 1.024 & 796.56 & $-2989,9129$ & 7.8 & 823.79 & $-6298,1890$ & 7.9 & 850.06 & $-42,4008$ & 6.8 & 1.33(19) & $<11$ \\
\hline $3 \mathrm{C} 216$ & 0.6703 & 965.23 & $\cdots$ & $\ldots$ & 998.24 & $-8938,2114$ & 1.5 & 1030.01 & $-3762,3223$ & 7.1 & $5.53(4)$ & $<0.57$ \\
\hline $0917+449$ & 2.1899 & 505.42 & $\ldots$ & RFI & 522.70 & $\ldots$ & RFI & 539.37 & $\cdots$ & RFI & $\ldots$ & $\cdots$ \\
\hline $0919-260$ & 2.3 & 488.55 & $\ldots$ & RFI & 505.26 & $-83,1934$ & 18 & 521.37 & $\ldots$ & RFI & $0.91(11)$ & $<42$ \\
\hline $0923+392$ & 0.6952 & 951.06 & $-1590,1090$ & 6.7 & 983.58 & $-9578,694$ & 5.7 & 1014.9 & $-5334,5004$ & 5.5 & $3.0(2)$ & $<4.1$ \\
\hline $0925-203$ & 0.34741 & 1196.5 & $-4257,3764$ & 4.5 & 1237.5 & $-3899,3857$ & 4.9 & 1276.9 & $-4849,4077$ & 3.1 & $0.83(8)$ & $<13$ \\
\hline HB89 0954+556 & 0.896 & 850.33 & $\ldots$ & 6.5 & 879.41 & & RFI & 907.45 & $\ldots$ & 6.1 & $4.20(16)^{\mathrm{a}}$ & $<26^{\mathrm{a}}$ \\
\hline $1004+141$ & 2.707 & 434.92 & $\ldots$ & RFI & 449.79 & & RFI & 464.13 & $-2629,2668$ & 8.9 & $1.243(13)^{\mathrm{b}}$ & $<147^{\mathrm{b}}$ \\
\hline $3 \mathrm{C} 237$ & 0.87 & 862.16 & & RFI & 891.64 & $-579,2310$ & 3.41 & 920.07 & $-1792,2049$ & 3.56 & $8.38(5)$ & $<0.95$ \\
\hline PKS 1034-293 & 0.312 & 1228.8 & $-4432,9474$ & 3.7 & 1270.9 & $-4753,3032$ & 5.6 & 1311.4 & $-5857,3287$ & 2.9 & $1.09(11)$ & $<11$ \\
\hline $1055+018$ & 0.888 & 853.94 & $-93,5524$ & 10.2 & 883.14 & & RFI & 911.30 & $-3883,4111$ & 8.3 & $3.668(13)^{\mathrm{a}}$ & $<61^{\mathrm{a}}$ \\
\hline $1116+128$ & 2.118 & 515.25 & & RFI & 532.87 & & RFI & 549.87 & & RFI & & \\
\hline PKS 1124-186 & 1.05 & 786.45 & $-6650,10351$ & 6 & 813.35 & $-7318,4845$ & 3.6 & 839.28 & $-7364,565$ & 4.2 & $0.48(4)$ & $<15$ \\
\hline B3 $1144+402$ & 1.088 & 772.14 & $-8060,5374$ & 5.1 & 798.54 & $-5277,6023$ & 4.5 & 824.01 & $-7855,4042$ & 6.8 & $0.5286(4)$ & $<17$ \\
\hline FBQS J1159+2914 & 0.7245 & 934.9 & $-5702,760$ & 6.4 & 966.87 & $-4430,5238$ & 5.6 & 997.70 & $-5694,7502$ & 7 & $2.12(3)$ & $<5.8$ \\
\hline $1202-262$ & 0.789 & 901.19 & $-8552,3324$ & 10.1 & 932.01 & $-7042,2189$ & 8.4 & 961.73 & $-4231,4217$ & 6.7 & $1.9640(9)$ & $<9.7$ \\
\hline $1222+037$ & 0.9556 & 824.42 & $-5048,3716$ & 5.6 & 852.61 & $-595,4925$ & 4.3 & 879.80 & & RFI & $0.94(11)^{\prime}$ & $<8.5$ \\
\hline PG $1222+216$ & 0.432 & 1125.9 & $\ldots$ & RFI & 1164.4 & $-6088,3182$ & 3.9 & 1201.45 & $-5118,3615$ & 3.3 & $1.90(9)$ & $<4.6$ \\
\hline $3 \mathrm{C} 273$ & 0.15834 & 1391.9 & $-3910,5567$ & 9.1 & 1439.4 & $-1158,3840$ & 7.1 & 1485.3 & $\cdots$ & $\cdots$ & $44.90(8)$ & $<0.34$ \\
\hline COINS J1244+4048 & 0.813 & 889.26 & $\ldots$ & RFI & 919.67 & $-1708,1731$ & 2.77 & 949.00 & $-2579,2558$ & 2.78 & $1.54(3)$ & $<4.1$ \\
\hline $3 \mathrm{C} 279$ & 0.5362 & 1049.5 & $-1859,5568$ & 4.7 & 1085.4 & & RFI & 1120.0 & $-538,1604$ & 4.6 & $10.6(2)^{\mathrm{a}}$ & $<7.9^{\mathrm{a}}$ \\
\hline $1308+326$ & 0.996 & 807.73 & $\ldots$ & RFI & 835.35 & $-4217,3894$ & 6.5 & 861.99 & $\ldots$ & RFI & $1.17(2)$ & $<11$ \\
\hline $3 \mathrm{C} 287$ & 1.055 & 784.54 & $-1719,1873$ & 3.98 & 811.37 & $-1315,2110$ & 4.33 & 837.24 & $-4930,419$ & 7.41 & $9.6(3)$ & $<0.87$ \\
\hline $3 \mathrm{C} 286$ & 0.8494 & 871.76 & & RFI & 901.57 & $-4224,3876$ & 6.55 & 930.32 & $\ldots$ & $\ldots$ & $18.04(9)$ & $<2.0$ \\
\hline B3 $1355+441$ & 0.646 & 979.48 & $-3635,2832$ & 5.61 & 1013.0 & $-3173,5706$ & 1.97 & 1045.3 & $-4136,3952$ & 1.92 & $0.58460(7)$ & $<7.0$ \\
\hline $1418+546$ & 0.1526 & 1398.8 & $-4120,6168$ & 3.2 & 1446.6 & & & 1492.7 & & & $0.92(7)$ & $<71^{\mathrm{a}}$ \\
\hline 3C309.1 & 0.905 & 846.32 & $-1603,3229$ & 9.63 & 875.25 & ... & RFI & 903.17 & $-3188,2305$ & 10.1 & $10.35(5)^{\mathrm{a}}$ & $<20^{\mathrm{a}}$ \\
\hline $1504-166$ & 0.876 & 859.40 & $-3070,6697$ & 7.7 & 888.78 & .. & RFI & 917.13 & $\ldots$ & $\ldots$ & $2.9(2)^{a}$ & $<58^{\mathrm{a}}$ \\
\hline 3C318 & 1.574 & 626.35 & & RFI & 647.77 & & RFI & 668.43 & & 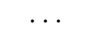 & & \\
\hline COINS J1546+0026 & 0.55 & 1040.2 & $-3473,4136$ & 3.3 & 1075.7 & $-2280,3517$ & 4.09 & 1110.0 & $-2290,707$ & 2.16 & $1.99(1)$ & $<4.0$ \\
\hline PKS $1622-253$ & 0.786 & 902.70 & $\cdots$ & RFI & 933.57 & & RFI & 963.34 & $\cdots$ & RFI & $\ldots$ & $\cdots$ \\
\hline PKS $1622-29$ & 0.815 & 888.28 & $\cdots$ & RFI & 918.66 & ... & RFI & 947.95 & $\ldots$ & RFI & $\ldots$ & $\ldots$ \\
\hline $3 \mathrm{C} 343$ & 0.988 & 810.98 & $-646,3490$ & 5.47 & 838.71 & $-4429,2920$ & 6.68 & 865.46 & $\ldots$ & $\ldots$ & $7.67(7)$ & $<1.6$ \\
\hline $1637+574$ & 0.7506 & 920.96 & $-567,4804$ & 3.6 & 952.45 & & & 982.82 & 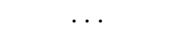 & & $0.9(3)^{a}$ & $<83^{\mathrm{a}}$ \\
\hline 3C 343.1 & 0.75 & 921.27 & $-744,1362$ & 5.87 & 952.78 & $-1425,1633$ & 8.71 & 983.16 & $-2476,3278$ & 9.95 & $6.39(5)$ & $<3.0$ \\
\hline $1642+690$ & 0.751 & 920.75 & $-505,6691$ & 4.8 & 952.23 & & & 982.60 & $\ldots$ & & $1.62(3)^{\mathrm{a}}$ & $<61^{\mathrm{a}}$ \\
\hline $1656+053$ & 0.879 & 858.03 & $-340,6438$ & 6.3 & 887.37 & $\cdots$ & RFI & 915.66 & $\ldots$ & RFI & $1.84(5)^{\mathrm{a}}$ & $<76^{\mathrm{a}}$ \\
\hline $1730-130$ & 0.902 & 847.65 & $-2458,2989$ & 9.5 & 876.63 & & RFI & 904.59 & $\ldots$ & RFI & $6.6(2)^{\mathrm{a}}$ & $<24^{\mathrm{a}}$ \\
\hline $1741-038$ & 1.054 & 784.92 & $-4078,7915$ & 10.6 & 811.76 & $-8139,5747$ & 5.6 & 837.65 & $-5995,662$ & 7.8 & $1.402(8)$ & $<7.8$ \\
\hline $1749+701$ & 0.77 & 910.86 & $-3895,5485$ & 4.8 & 942.01 & & $\ldots$ & 972.05 & $\ldots$ & $\ldots$ & $1.3(7)^{\mathrm{a}}$ & $<76^{\mathrm{a}}$ \\
\hline COINS J1815+6127 & 0.601 & 1007.0 & $-957,1428$ & 2.09 & 1041.5 & $-2672,1721$ & 2.07 & 1074.7 & $-800,234$ & 3.19 & $0.50(5)$ & $<8.4$ \\
\hline $1823+568$ & 0.664 & 968.89 & $-1643,6711$ & 4.6 & 1002.0 & $-4542,3027$ & 5.3 & 1034.0 & & RFI & $1.62(5)$ & $<6.9$ \\
\hline 3C380 & 0.69 & 953.98 & $-197,2084$ & 3.52 & 985.44 & $-2666,1776$ & 4.6 & 1018.1 & $-1954,1874$ & 5.83 & $18.89(17)$ & $<0.52$ \\
\hline $4 \mathrm{C}+29.56$ & 0.842 & 875.26 & $\ldots$ & RFI & 905.19 & & RFI & 934.06 & & $\ldots$ & $\ldots$ & $\ldots$ \\
\hline WMAP J1849+6705 & 0.657 & 972.98 & $-6507,7328$ & 4.7 & 1006.3 & $-3798,5736$ & 5.8 & 1038.3 & $-479,676$ & 4.9 & $0.6(1.3)$ & $<20$ \\
\hline TXS $1848+283$ & 2.56 & 452.87 & $-461,5279$ & 13.6 & 468.36 & & RFI & 483.29 & & $\ldots$ & $0.188(6)^{\mathrm{a}}$ & $<1640^{\mathrm{a}}$ \\
\hline PKS $2000-330$ & 3.7730 & 337.78 & $-7046,3614$ & 11.5 & 349.33 & $-3587,4514$ & 11.6 & 360.47 & $-4066,4234$ & 9.19 & $0.18(2)$ & $<109$ \\
\hline PKS 2008-068 & 0.5470 & 1042.2 & $-240,2435$ & 6.67 & 1077.8 & & RFI & 1112.2 & & $\ldots$ & $2.307(10)^{\mathrm{a}}$ & $<51^{\mathrm{a}}$ \\
\hline COINS J2022+6136 & 0.227 & 1314.0 & $-5713,4098$ & 4.8 & 1358.9 & $-2892,7256$ & 4.3 & 1402.2 & $-3800,7959$ & 4.3 & $2.18(17)$ & $<4.6$ \\
\hline $2059+034$ & 1.013 & 800.91 & $-5387,6704$ & 6.4 & 828.30 & $-3730,6550$ & 4.8 & 854.71 & $-698,6352$ & 5.6 & $0.342(3)$ & $<27$ \\
\hline PKS 2126-15 & 3.2680 & 377.75 & $-4366,3110$ & 5.62 & 390.67 & $-4405,2563$ & 8.47 & 403.12 & $\ldots$ & ... & $0.124(8)$ & $<204$ \\
\hline PKS $2127+04$ & 0.99 & 810.17 & $-1633,2659$ & 6.08 & 837.87 & $-4812,5707$ & 8.27 & 864.59 & $\ldots$ & RFI & $4.80(6)$ & $<3.2$ \\
\hline
\end{tabular}


Table 3 - Continued

\begin{tabular}{|c|c|c|c|c|c|c|c|c|c|c|c|c|}
\hline \multirow[b]{2}{*}{ Source } & \multirow[b]{2}{*}{$z$} & \multicolumn{3}{|c|}{$1612 \mathrm{MHz}$} & \multicolumn{3}{|c|}{$1667 \mathrm{MHz}$} & \multicolumn{3}{|c|}{$1720 \mathrm{MHz}$} & \multirow[b]{2}{*}{$\begin{array}{l}\text { Cntm } \\
(\mathrm{Jy})\end{array}$} & \multirow[b]{2}{*}{$\begin{array}{c}N_{\mathrm{OH}} / T_{x} \\
\left(10^{13} \mathrm{~cm}^{-2} \mathrm{~K}^{-1}\right)\end{array}$} \\
\hline & & $\begin{array}{c}\nu \\
(\mathrm{MHz})\end{array}$ & $\begin{array}{c}\Delta v \\
\left(\mathrm{~km} \mathrm{~s}^{-1}\right)\end{array}$ & $\begin{array}{l}\mathrm{rms} \\
(\mathrm{mJy})\end{array}$ & $\begin{array}{c}\nu \\
(\mathrm{MHz})\end{array}$ & $\begin{array}{c}\Delta v \\
\left(\mathrm{~km} \mathrm{~s}^{-1}\right)\end{array}$ & $\begin{array}{l}\mathrm{rms} \\
(\mathrm{mJy})\end{array}$ & $\begin{array}{c}\nu \\
(\mathrm{MHz})\end{array}$ & $\begin{array}{c}\Delta v \\
\left(\mathrm{~km} \mathrm{~s}^{-1}\right)\end{array}$ & $\begin{array}{l}\mathrm{rms} \\
(\mathrm{mJy})\end{array}$ & & \\
\hline $2128-123$ & 0.501 & 1074.1 & $\ldots$ & RFI & 1110.8 & & RFI & 1146.2 & $\ldots$ & RFI & 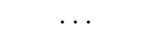 & $\ldots$ \\
\hline PKS 2135-209 & 0.63634 & 985.27 & $-2832,2795$ & 4.34 & 1019.0 & $-1620,4348$ & 8.22 & 1051.5 & $\cdots$ & RFI & $4.9(6)$ & $<3.4$ \\
\hline $2145+067$ & 0.990 & 810.17 & $-4601,4,317$ & 5.7 & 837.87 & $-4663,597$ & 5.2 & 864.59 & $\cdots$ & RFI & $3.304(2)$ & $<3.0$ \\
\hline $2155-152$ & 0.672 & 964.25 & $-2564,5364$ & 6.1 & 997.22 & $-9252,5599$ & 5.1 & 1029.0 & $-6693,9330$ & 3.9 & $2.79(18)$ & $<3.8$ \\
\hline $2201+315$ & 0.295 & 1245.0 & $-2175,3604$ & 5.3 & 1287.5 & $-3368,6877$ & 5.2 & 1328.6 & $-3025,6452$ & 3.6 & $1(3)$ & $<5.6$ \\
\hline $2216-038$ & 0.901 & 848.10 & $-956,3852$ & 6.3 & 877.10 & & RFI & 905.07 & & RFI & $1.3460(3)^{\mathrm{a}}$ & $<78^{\mathrm{a}}$ \\
\hline PKS $2230+11$ & 1.037 & 791.47 & $-1321,1342$ & 5.06 & 818.54 & $-3388,3392$ & 12.2 & 844.64 & $-2591,1671$ & 4.44 & $7.34(7)$ & $<2.1$ \\
\hline $2234+282$ & 0.795 & 898.18 & & RFI & 928.89 & $-3618,6097$ & 5.6 & 958.51 & $-4469,3851$ & 4.5 & $0.626(6)$ & $<20$ \\
\hline $3 \mathrm{C} 454$ & 1.757 & 584.78 & $\cdots$ & RFI & 604.77 & - & RFI & 624.06 & $\ldots$ & $\cdots$ & $\ldots$ & $\cdots$ \\
\hline $3 \mathrm{C} 454.3$ & 0.859 & 867.26 & $\cdots$ & RFI & 896.91 & .. & RFI & 925.51 & $\cdots$ & $\cdots$ & $\cdots$ & $\cdots$ \\
\hline $3 \mathrm{C} 455$ & 0.543 & 1044.9 & $\cdots$ & RFI & 1080.6 & .. & RFI & 1115.1 & $\cdots$ & $\cdots$ & $\ldots$ & $\ldots$ \\
\hline $2255-282$ & 0.92548 & 837.31 & 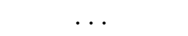 & RFI & 865.94 & 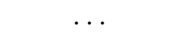 & RFI & 893.56 & $-9961,1785$ & 4.4 & $1.1440(5)^{\mathrm{b}}$ & $<81^{\mathrm{b}}$ \\
\hline $3 \mathrm{C} 459$ & 0.22012 & 1321.4 & $-4113,5643$ & 4.4 & 1366.6 & $-1546,8940$ & 4.2 & 1410.1 & $-2055,3132$ & 2.9 & $4.50(3)$ & $<2.1$ \\
\hline HB89 $2342+821$ & 0.735 & 929.24 & $-3036,3539$ & 4.3 & 961.01 & $-4314,3017$ & 6.12 & 991.66 & $-3579,2343$ & 4.7 & $4.32(4)$ & $<3.0$ \\
\hline HB89 2344+092 & 0.677 & 961.38 & $-3281,4421$ & 4.6 & 994.25 & $-5352,4267$ & 4.4 & 1026.0 & $-2350,7585$ & 4.2 & $2.198(2)$ & $<4.2$ \\
\hline
\end{tabular}

Note. - Columns list the source name, the optical redshift, and the expeted frequency, the veloctity range searched (blue, red limits), and the spectral rms noise for each of the 1612,1667 , and $1720 \mathrm{MHz} \mathrm{OH}$ lines. The final two columns list the continuum flux density expected at the redshifted $1667 \mathrm{MHz} \mathrm{OH}$ line, and the $3 \sigma$ column density limit for that line, unless otherwise noted (Sec. 4.3. Numbers in parentheses show uncertainties in the final digit(s).

a Since the 1667 (and 1665) MHz line was not observable due to RFI, we use the $1612 \mathrm{MHz}$ line to obtain an upper limit on the OH column density. The interpolated continuum corresponds to the redshifted frequency of this line.

bhe interpolated continuum and $\mathrm{OH}$ column density limit are based on the $1720 \mathrm{MHz} \mathrm{OH}$ line. 
Table 4

Intrinsic HI $21 \mathrm{~cm}$ Absorption Line Measurements and Derived Quantities

\begin{tabular}{|c|c|c|c|c|c|c|c|c|c|}
\hline Source & Comp. & $\begin{array}{c}\nu \\
(\mathrm{MHz})\end{array}$ & $z$ & $\begin{array}{c}\text { Ctnm } \\
\text { (Jy) }\end{array}$ & $\begin{array}{c}\text { FWHM } \\
(\mathrm{kHz})\end{array}$ & $\begin{array}{c}\text { FWHM } \\
\left(\mathrm{km} \mathrm{s}^{-1}\right)\end{array}$ & $\begin{array}{l}\text { Depth } \\
\text { (mJy) }\end{array}$ & $\begin{array}{c}\tau \\
\left(10^{-2}\right)\end{array}$ & $\begin{array}{c}N_{\mathrm{HI}} \\
\left(10^{20} \mathrm{~cm}^{-2}\right)\end{array}$ \\
\hline \multirow[t]{3}{*}{ COINS J0111+3906 } & 1 & $851.373(5)$ & $0.668371(10)$ & $0.153(2)$ & 190(10) & $67(4)$ & $85(2)$ & $81_{-4}^{+2}$ & $104_{-8}^{+7}$ \\
\hline & 2 & $851.173(9)$ & $0.668763(18)$ & $0.153(2)$ & $142(17)$ & $50(6)$ & $34(3)$ & $25_{-3}^{+2}$ & $24_{-4}^{+3}$ \\
\hline & Tot & 851.3278 & 0.6685 & $0.153(2)$ & $180(20)$ & $63(7)$ & $70(8)$ & $61(12)$ & $74(13)$ \\
\hline \multirow[t]{3}{*}{$4 \mathrm{C}+76.03$} & 1 & $887.643(3)$ & $0.600199(5)$ & $6.618(7)$ & $132(10)$ & $45(3)$ & $114(8)$ & $1.74_{-0.12}^{+0.12}$ & $1.48_{-0.15}^{+0.14}$ \\
\hline & 2 & $887.69(3)$ & $0.60011(5)$ & $6.618(7)$ & $559(90)$ & $189(29)$ & $36(6)$ & $0.53_{-0.10}^{+0.09}$ & $1.9_{-0.5}^{+0.4}$ \\
\hline & Tot & 887.648 & 0.60019 & $6.618(7)$ & $199(79)$ & $67.2(27)$ & $165(6)$ & $2.9(1.1)$ & $3.7(1.1)$ \\
\hline \multirow[t]{3}{*}{ PKS 0500+019 } & 1 & $896.324(9)$ & $0.58470(2)$ & $1.57(7)$ & $284(20)$ & $95(7)$ & $49(3)$ & $3.2_{-0.3}^{+0.1}$ & $5.9_{-0.7}^{+0.5}$ \\
\hline & 2 & $895.878(9)$ & $0.58549(2)$ & $1.57(7)$ & $77(21)$ & $26(7)$ & $25(6)$ & $1.6_{-0.4}^{+0.3}$ & $0.79_{-0.3}^{+0.2}$ \\
\hline & Tot & 896.299 & 0.5847 & $1.57(7)$ & $372(47)$ & $125(16)$ & $58(7)$ & $3.0(4)^{-0}$ & $7.1(1.3)$ \\
\hline \multirow[t]{3}{*}{$3 \mathrm{C} 216$} & 1 & $850.16(2)$ & $0.67075(4)$ & $6.39(4)$ & $214(47)$ & $75(20)$ & $12(3)$ & $0.19_{-0.05}^{+0.04}$ & $0.28_{-0.10}^{+0.09}$ \\
\hline & 2 & $850.37(3)$ & $0.67034(6)$ & $6.39(4)$ & $701(43)$ & $247(15)$ & $23.4(1.9)$ & $0.37_{-0.03}^{+0.02}$ & $1.74_{-0.19}^{+0.17}$ \\
\hline & Tot & 850.336 & 0.6704 & $6.39(4)$ & $613(95)$ & $216(33)$ & $37(4)$ & $0.44(7)$ & $1.8(4)$ \\
\hline \multirow[t]{3}{*}{ COINS J1815+6127 } & 1 & $885.945(8)$ & $0.603266(14)$ & $0.53(6)$ & $219(19)$ & $71(6)$ & $21.9(1.5)$ & $4.21_{-0.08}^{+0.03}$ & $5.7_{-1.2}^{+0.7}$ \\
\hline & 2 & $886.24(2)$ & $0.60273(4)$ & $0.53(6)$ & $167(56)$ & $56(19)$ & $6.6(6)$ & $1.250_{-0.02}^{+0.011}$ & $1.4_{-0.5}^{+0.5}$ \\
\hline & Tot & 885.978 & 0.6032 & 0.53 & $296(88)$ & $100(30)$ & $25(3)$ & $3.7(1.1)$ & $7(3)$ \\
\hline \multirow[t]{4}{*}{$3 \mathrm{C} 455$} & 1 & $920.51(1)$ & $0.54313(3)$ & $4.45(5)$ & $309(47)$ & $101(15)$ & $9.2(1.4)$ & $0.21_{-0.04}^{+0.03}$ & $0.40_{-0.09}^{+0.08}$ \\
\hline & 2 & $920.34(3)$ & $0.54335(5)$ & $4.45(5)$ & $829(60)$ & $270(20)$ & $10.3(1.4)$ & $0.23_{-0.03}^{+0.02}$ & $1.20_{-0.19}^{+0.16}$ \\
\hline & 3 & $919.55(1)$ & $0.54467(2)$ & $4.45(5)$ & $153(28)$ & $50(9)$ & $6.5(9)$ & $0.15_{-0.02}^{+0.01}$ & $0.14_{-0.03}^{+0.03}$ \\
\hline & Tot & 920.376 & 0.5433 & $4.45(5)$ & $510(150)$ & $170(50)$ & $20(2)$ & $0.57(17)$ & $1.8(6)$ \\
\hline
\end{tabular}

Note. - Sources for which $21 \mathrm{~cm}$ absorption is detected. Columns list the source name, Gaussian component, central frequency, redshift, continuum flux density, FWHM width of the line (rest-frame), line depth, peak optical depth, and derived column density corresponding to each individual Gaussian component. For rows labeled with Tot, these measurements correspond to the direct integration for each absorption profile. Numbers in parentheses show statistical uncertainties in the final digit(s).

Table 5

UV, Optical, Near-IR, and Radio Properties

\begin{tabular}{|c|c|c|c|c|c|c|c|c|c|}
\hline Source & $\begin{array}{c}B \\
(\mathrm{mag})\end{array}$ & $\begin{array}{c}V \\
(\mathrm{mag})\end{array}$ & $\begin{array}{c}R \\
(\mathrm{mag})\end{array}$ & $\begin{array}{c}K \\
(\mathrm{mag})\end{array}$ & $z$ & $\begin{array}{c}D_{\mathrm{L}} \\
(\mathrm{Mpc})\end{array}$ & $\begin{array}{c}\log L_{\mathrm{UV}} \\
\left(\mathrm{W} \mathrm{Hz}{ }^{-1}\right)\end{array}$ & $\begin{array}{l}\log L_{\text {Radio }} \\
\left(\mathrm{W} \mathrm{Hz}^{-1}\right)\end{array}$ & References \\
\hline $0018+729$ & $\ldots$ & $\ldots$ & 20.9 (i-band) & . & 0.821 & 5188.7 & . & 27.25 & 1 \\
\hline COINS J0111+3906 & $\ldots$ & $\cdots$ & 22.00 & 16.69 & 0.66847 & 4023.5 & 20.40 & 26.21 & 2,3 \\
\hline UM321 & 16.72 & 16.44 & 16.30 & 14.41 & 1.07481 & 7303.7 & 24.39 & 27.60 & $4,5,6$ \\
\hline $3 \mathrm{C} 43$ & 21.38 & $\ldots$ & 19.70 & 20.0 & 1.459 & 10635 & 22.27 & 28.52 & 7,8 \\
\hline $3 \mathrm{C} 48$ & 16.62 & 16.2 & & 12.72 & 0.367 & 1950.7 & 23.07 & 27.83 & 7,6 \\
\hline $0138-097$ & 17.1 & $\cdots$ & 17.0 & 14.40 & 0.733 & 4508.2 & 24.13 & 27.03 & 10,6 \\
\hline PKS $0201+113$ & $\ldots$ & $\ldots$ & 19.5 & 16.8 & 3.6390 & 32616 & 24.08 & 27.96 & 11 \\
\hline B2 $0218+357$ & $\ldots$ & 19.60 & & 14.84 & 0.944 & 6173.8 & 22.38 & 27.56 & 5,6 \\
\hline $3 \mathrm{C} 066 \mathrm{~A}$ & $\ldots$ & $\ldots$ & 12.85 (H-band) & 12.02 & 0.444 & 2446.5 & 23.75 & 27.15 & 12 \\
\hline $0221+067$ & 19.65 & 18.97 & 17.78 & 14.41 & 0.511 & 2897.7 & 21.18 & 26.81 & $8,5,13$ \\
\hline HB89 $0235+164$ & $\ldots$ & 19.0 & 16.89 & 10.49 & 0.940 & 6141.2 & 19.02 & 27.39 & $14,15,16$ \\
\hline PKS 0237-23 & $\ldots$ & 16.6 & $\ldots$ & 13.6 & 2.223 & 17922 & 24.88 & 28.61 & 17,18 \\
\hline $0306+102$ & $\cdots$ & 21.2 & 19.91 & 15.19 & 0.863 & 5520.8 & 19.78 & 26.98 & $19,5,6$ \\
\hline NGC 1275 & 14.80 & 13.03 & 12.62 & 11.30 & 0.01765 & 75.6 & 19.06 & 25.19 & $20,21,22,6$ \\
\hline $\mathrm{OE}+131$ & $\ldots$ & 19.0 & 19.0 & 15.38 & 2.662 & 22347 & 24.27 & 28.64 & $7,18,6$ \\
\hline PKS 0327-241 & $\ldots$ & $\ldots$ & 19.31 & $\ldots$ & 0.895 & 5776.9 & $\ldots$ & 27.30 & 5 \\
\hline $0336-019$ & 18.32 & 17.77 & 17.33 & 14.49 & 0.852 & 5433.4 & 23.13 & 27.63 & $4,5,6$ \\
\hline $0346-279$ & $\ldots$ & $\ldots$ & 17.72 & 14.68 & 0.991 & 6559.7 & 23.38 & 27.43 & 5,23 \\
\hline $4 C+76.03$ & $\ldots$ & $\ldots$ & 21.2 & $\ldots$ & 0.5985 & 3512.8 & $\ldots$ & 27.79 & 24 \\
\hline 3C119 & $\ldots$ & $\ldots$ & 20.0 & 15.02 & 1.023 & 6825.3 & 21.91 & 28.59 & 7,6 \\
\hline $0440-003$ & 18.35 & 17.98 & 17.85 & $\ldots$ & 0.844 & 5370.0 & 23.46 & 27.65 & 4,5 \\
\hline PKS $0457+024$ & 18.78 & 18.5 & $\ldots$ & $\ldots$ & 2.384 & 19528 & 24.23 & 27.72 & 4 \\
\hline PKS $0500+019$ & 22.5 & 21.35 & 20.68 & 15.43 & 0.58457 & 3413.1 & 20.28 & 27.14 & $25,26,27$ \\
\hline $3 \mathrm{C} 138$ & 19.37 & 18.84 & 18.48 & 15.43 & 0.76 & 4714.7 & 22.65 & 28.24 & $28,29,6$ \\
\hline PKS 0528-250 & 18.16 & $\cdots$ & $\cdots$ & 15.37 & 2.813 & 23900 & 24.63 & 28.44 & 30 \\
\hline PKS $0539-057$ & & 19.9 & & 14.41 & 0.839 & 5330.5 & 21.93 & 27.23 & 31,6 \\
\hline $3 \mathrm{C} 147$ & 18.45 & 17.8 & 17.21 & 14.56 & 0.55 & 3168.8 & 22.33 & 28.36 & $28,49,6$ \\
\hline B3 $0552+398$ & 18.62 & 18.0 & 18.03 & 14.68 & 2.365 & 19338 & 24.29 & 27.81 & $8,18,6$ \\
\hline $0605-085$ & & 18.5 & 17.70 & 12.41 & 0.872 & 5592.6 & 22.02 & 27.74 & $14,5,6$ \\
\hline HB89 $0636+680$ & 17.59 & $\ldots$ & 16.58 & 13.75 & 3.1800 & 27729 & 25.04 & 27.41 & 8,6 \\
\hline B2 $0711+35$ & $\ldots$ & 17.0 & $\ldots$ & $\ldots$ & 1.62 & 12116 & $\ldots$ & 27.77 & 18 \\
\hline $0735+178$ & 16.28 & 15.65 & 15.29 & 10.34 & 0.424 & 2315.3 & 23.14 & 26.99 & 32,33 \\
\hline
\end{tabular}


Intrinsic $\mathrm{HI}$ and $\mathrm{OH}$ Absorption in Compact Radio Sources

Table 5 - Continued

\begin{tabular}{|c|c|c|c|c|c|c|c|c|c|}
\hline Source & $\begin{array}{c}B \\
(\mathrm{mag})\end{array}$ & $\begin{array}{c}V \\
(\mathrm{mag})\end{array}$ & $\begin{array}{c}R \\
(\mathrm{mag})\end{array}$ & $\begin{array}{c}K \\
(\mathrm{mag})\end{array}$ & $z$ & $\begin{array}{c}D_{\mathrm{L}} \\
(\mathrm{Mpc})\end{array}$ & $\begin{array}{c}\log L_{\mathrm{UV}} \\
\left(\mathrm{W} \mathrm{Hz} z^{-1}\right)\end{array}$ & $\begin{array}{l}\log L_{\text {Radio }} \\
\left(\mathrm{W} \mathrm{Hz}^{-1}\right)\end{array}$ & References \\
\hline $0736+017$ & 14.90 & 14.34 & 13.39 & 12.96 & 0.189410 & 921.8 & 22.23 & 26.33 & 34,35 \\
\hline B2 $0738+31$ & 16.52 & 16.1 & 16.32 & 16.1 & 0.6314 & 3750.9 & 24.16 & 27.19 & $8,36,37$ \\
\hline PKS $0742+10$ & 24 & 23.8 & 23.1 & $\ldots$ & 2.624 & 21857 & 22.23 & 28.39 & 38 \\
\hline $3 \mathrm{C} 186$ & 18.50 & 17.97 & 17.67 & 15.68 & 1.0670 & 7194 & 23.42 & 27.95 & 32,41 \\
\hline PKS $0745+241$ & 19.65 & 18.80 & 18.31 & 13.90 & 0.4092 & 2219.3 & 21.4 & 26.64 & 32,6 \\
\hline COINS J0753+4231 & 19.44 & 18.26 & 17.59 & 15.47 & 3.5892 & 32081 & 24.66 & 28.31 & 32,42 \\
\hline HB89 $0754+100$ & 15.76 & 15.22 & 14.91 & 11.71 & 0.2660 & 1341.3 & 23.01 & 26.25 & 32,33 \\
\hline PKS $0823+033$ & 17.95 & 17.32 & 16.96 & 14.42 & 0.506 & 2863.6 & 22.67 & 26.92 & 32,6 \\
\hline PKS $0823-223$ & $\cdots$ & 16.2 & 15.4 & 12.19 & 0.910 & 5897.8 & 23.01 & 27.15 & $36,39,40$ \\
\hline $0828+493$ & 20.46 & 19.61 & 19.12 & 14.83 & 0.548 & 3154.5 & 21.44 & 26.89 & 32,6 \\
\hline PKS $0858-279$ & $\ldots$ & 17.0 & & 13.59 & 2.152 & 17220 & 24.65 & 27.99 & 18,6 \\
\hline TXS $0902+490$ & 17.74 & 17.49 & 17.35 & 15.08 & 2.6887 & 22621 & 24.71 & 28.20 & 32,6 \\
\hline $3 \mathrm{C} 216$ & 18.96 & 19.05 & 18.22 & 14.86 & 0.6703 & 4037.1 & 22.79 & 27.87 & $43,32,41$ \\
\hline $0919-260$ & 18.05 & $\ldots$ & 18.01 & 15.04 & 2.300 & 18688 & 24.54 & 28.04 & 30,5 \\
\hline $0923+392$ & 17.07 & 17.02 & 16.79 & 14.16 & 0.6952 & 4222.7 & 23.96 & 27.60 & 12 \\
\hline $0925-203$ & $\ldots$ & $\cdots$ & 16.00 & 13.78 & 0.34741 & 1832.4 & 23.32 & 26.42 & 5,35 \\
\hline HB89 0954+556 & 18.30 & 17.71 & 17.38 & 14.19 & 0.896 & 5784.9 & 23.24 & 27.96 & 32,6 \\
\hline HB89 0954+658 & $\ldots$ & $\ldots$ & 15.75 & 12.43 & 0.368 & 1957.0 & 22.99 & 26.41 & 46,40 \\
\hline $1004+141$ & 18.97 & 18.53 & 18.28 & $\ldots$ & 2.707 & 22808 & 24.28 & 28.34 & 32 \\
\hline $3 \mathrm{C} 237$ & 21.49 & 21.14 & 20.95 & $\ldots$ & 0.87 & 5576.6 & 20.89 & 28.27 & 32 \\
\hline PKS 1034-293 & 18.83 & 18.26 & 17.54 & 12.71 & 0.312 & 1612.8 & 21.43 & 26.4 & $13,5,33$ \\
\hline $1055+018$ & 18.19 & 17.73 & 16.68 & 14.12 & 0.888 & 5720.7 & 22.87 & 27.89 & $4,5,6$ \\
\hline PKS 1124-186 & & & 19.23 & 12.28 & 1.050 & 7051.1 & 21.65 & 27.13 & 5,6 \\
\hline B3 $1144+402$ & 18.97 & 18.25 & 18.12 & 13.53 & 1.0880 & 7371.3 & 23.24 & 27.27 & 32,6 \\
\hline FBQS J1159+2914 & 18.67 & 18.12 & 17.93 & 11.47 & 0.7245 & 4450.9 & 23.00 & 27.49 & 32,6 \\
\hline $1202-262$ & & & $15.97(\mathrm{H}-$ band $)$ & 15.04 & 0.789 & 4938.8 & 22.85 & 27.56 & 6 \\
\hline B2 $1219+28$ & 15.72 & 15.31 & 14.72 & 11.85 & 0.102 & 464.6 & 21.89 & 25.33 & 32,15 \\
\hline $1222+037$ & 19.23 & 18.79 & 18.5 & $\ldots$ & 0.9556 & 6268.6 & 23.07 & 27.31 & 4,10 \\
\hline PG $1222+216$ & 15.976 & 15.98 & 15.99 & 13.07 & 0.4320 & 2367.6 & 24.27 & 26.98 & 32,6 \\
\hline $3 \mathrm{C} 273$ & 12.99 & 12.81 & 12.71 & 9.81 & 0.15834 & 748.7 & 24.28 & 27.44 & $12,15,35$ \\
\hline COINS J1244+4048 & 20.68 & 20.22 & 19.97 & & 0.813 & 5126 & 21.16 & 27.45 & 32 \\
\hline $3 \mathrm{C} 279$ & 18.01 & 17.75 & 17.11 & 12.58 & 0.5362 & 3072.1 & 22.79 & 27.91 & 28,16 \\
\hline $3 \mathrm{C} 287$ & 18.67 & 18.12 & 17.80 & 15.50 & 1.055 & 7093 & 23.32 & 28.49 & 32,6 \\
\hline $3 \mathrm{C} 286$ & 17.51 & 17.25 & 16.5 & 14.88 & 0.8494 & 5412.8 & 25.67 & 28.57 & $44,45,10,6$ \\
\hline HB89 1333+459 & 18.00 & 17.91 & 17.89 & 15.04 & 2.4490 & 20183 & 24.55 & 27.16 & 32,6 \\
\hline $1354+195$ & 16.34 & 16.09 & 15.92 & 13.89 & 0.720 & 4409.6 & 24.17 & 27.63 & 32,6 \\
\hline $1418+546$ & 16.27 & 15.63 & 15.27 & 11.43 & 0.1526 & 718.9 & 22.06 & 25.68 & 32,6 \\
\hline 3С 309.1 & 16.4 & & & 14.72 & 0.905 & 5857.4 & 24.45 & 28.39 & 10,6 \\
\hline $1504-166$ & 20.28 & 19.75 & 17.00 & 14.01 & 0.876 & 5624.6 & 20.77 & 27.72 & $13,31,8$ \\
\hline 3C318 & 21.14 & 20.18 & 19.64 & $\cdots$ & 1.5740 & 11689 & 22.38 & 28.6 & 32 \\
\hline COINS J1546+0026 & 19.73 & 18.9 & 20.1 & 15.43 & 0.55 & 3168.5 & 23.28 & 27.2 & 4,6 \\
\hline PKS $1622-253$ & 20.95 & $\ldots$ & 19.60 & 14.86 & 0.786 & 4915.5 & 21.27 & 27.6 & $8,5,6$ \\
\hline PKS $1622-29$ & $\ldots$ & $\ldots$ & $15.06($ H-band $)$ & 14.15 & 0.815 & 5141.6 & 23.30 & 27.69 & 6 \\
\hline 3C 343 & 21.26 & $\ldots$ & 20.6 & & 0.988 & 6534.9 & 22.34 & 28.34 & 8,18 \\
\hline $1637+574$ & 17.11 & 16.9 & 16.94 & 13.54 & 0.7506 & 4642.6 & 24.09 & 27.14 & $8,31,5,6$ \\
\hline 3C343.1 & $\cdots$ & $\cdots$ & 20.8 & $\cdots$ & 0.75 & 4638.0 & $\cdots$ & 28.03 & 18 \\
\hline $1642+690$ & $\ldots$ & 19.2 & 19.38 & & 0.751 & 4642.6 & 22.54 & 27.39 & 14,5 \\
\hline $1656+053$ & 17.33 & 16.55 & 16.21 & 14.06 & 0.879 & 5648.5 & 23.88 & 27.59 & $13,47,5,35$ \\
\hline $1730-130$ & $\ldots$ & 19.5 & 18.78 & 14.29 & 0.902 & 5833.2 & 23.05 & 28.16 & $31,5,6$ \\
\hline $1749+701$ & $\ldots$ & 17.0 & 15.48 & 13.03 & 0.770 & 4791.7 & 24.21 & 27.31 & $14,5,40$ \\
\hline $1803+784$ & $\cdots$ & 15.9 & 15.46 & 14.15 & 0.680 & 4109.2 & 24.51 & 27.4 & $31,5,48$ \\
\hline COINS J1815+6127 & 21.27 & $\cdots$ & 19.12 & $\cdots$ & 0.601 & 3530.8 & 20.53 & 26.69 & 8 \\
\hline 3C380 & 16.26 & $\ldots$ & 16.86 & 14.25 & 0.69 & 4183.8 & 24.99 & 28.43 & $8,29,41$ \\
\hline $4 C+29.56$ & 21.92 & $\ldots$ & 20.2 & & 0.842 & 5354.2 & 21.15 & 27.86 & 8 \\
\hline TXS $1848+283$ & 18.19 & 18.09 & 17.12 & 14.62 & 2.56 & 21307 & 24.52 & 26.42 & $49,8,6$ \\
\hline PKS 2000-330 & 18.34 & 17.05 & 16.92 & 15.10 & 3.7730 & 34060 & 25.31 & 27.66 & 30,13 \\
\hline $2007+777$ & $\ldots$ & $\ldots$ & 17.36 & 13.83 & 0.342 & 1795.4 & 22.19 & 26.46 & 40,5 \\
\hline PKS 2008-068 & $\cdots$ & $\cdots$ & 21.3 & $\cdots$ & 0.5470 & 3147.5 & $\cdots$ & 27.21 & 7 \\
\hline COINS J2022+6136 & 19.83 & $\cdots$ & 17.58 & 14.28 & 0.227 & 1119.4 & 19.59 & 26.39 & $8,5,6$ \\
\hline PKS 2121-01 & $\cdots$ & $\ldots$ & 23.3 & 18.18 & 1.158 & 7968.8 & 20.72 & 27.74 & 50 \\
\hline PKS 2126-15 & 17.63 & 16.92 & 16.63 & 14.25 & 3.2680 & 28658 & 25.18 & 27.37 & 30,13 \\
\hline PKS 2127+04 & & & 22.21 & & 0.99 & 6551.5 & & 28.11 & 50 \\
\hline $2128-123$ & 16.15 & 15.97 & 15.79 & 13.33 & 0.501 & 2829.4 & 23.97 & 27.06 & 12 \\
\hline $2145+067$ & 16.63 & 16.25 & 15.06 & 13.48 & 0.990 & 6551.5 & 23.61 & 27.94 & $4,5,13$ \\
\hline PKS 2149+056 & 23.7 & 22.05 & 20.85 & 17.17 & 0.740 & 4561.5 & 19.35 & 26.86 & 13 \\
\hline $2155-152$ & 18.42 & 18.3 & 17.79 & 14.46 & 0.672 & 4049.7 & 23.20 & 27.5 & $15,31,5,33$ \\
\hline $2201+315$ & 15.52 & 15.46 & 14.33 & 12.34 & 0.2950 & 1511.2 & 22.83 & 26.61 & $8,31,5,6$ \\
\hline $2216-038$ & 17.36 & 16.81 & 16.15 & 14.07 & 0.901 & 5825.2 & 23.43 & 27.47 & $4,5,35$ \\
\hline PKS $2230+11$ & 17.75 & 17.33 & & 13.77 & 1.037 & 6942.2 & 23.72 & 28.33 & 45,6 \\
\hline $2234+282$ & 18.94 & $\ldots$ & 17.60 & 14.87 & 0.795 & 4985.4 & 22.53 & 27.0 & $8,5,6$ \\
\hline $3 \mathrm{C} 454$ & 18.64 & 18.52 & $\cdots$ & $\cdots$ & 1.757 & 13402 & 24.09 & 28.58 & 4 \\
\hline 3C454.3 & 16.17 & 15.70 & 15.22 & 12.95 & 0.859 & 5489.0 & 24.02 & 28.43 & $4,5,33$ \\
\hline $3 \mathrm{C} 455$ & 19.54 & 19.59 & 19.19 & 13.00 & 0.543 & 3119.5 & 22.69 & 27.53 & $8,32,29,6$ \\
\hline $3 \mathrm{C} 459$ & 18.31 & 17.44 & 16.78 & 13.99 & 0.22012 & 1081.1 & 21.00 & 26.78 & $51,52,6$ \\
\hline HB89 $2342+821$ & 21.77 & & 20.22 & 15.85 & 0.735 & 4523.4 & 21.13 & 27.81 & $8,24,53$ \\
\hline
\end{tabular}


Table 5 - Continued

\begin{tabular}{|c|c|c|c|c|c|c|c|c|c|}
\hline Source & $\begin{array}{c}B \\
(\mathrm{mag})\end{array}$ & $\begin{array}{c}V \\
(\mathrm{mag})\end{array}$ & $\begin{array}{c}R \\
(\mathrm{mag})\end{array}$ & $\begin{array}{c}K \\
(\mathrm{mag})\end{array}$ & $z$ & $\begin{array}{c}D_{\mathrm{L}} \\
(\mathrm{Mpc})\end{array}$ & $\begin{array}{c}\log L_{\mathrm{UV}} \\
\left(\mathrm{W} \mathrm{Hz}^{-1}\right)\end{array}$ & $\begin{array}{l}\log L_{\text {Radio }} \\
\left(\mathrm{W} \mathrm{Hz}^{-1}\right)\end{array}$ & References \\
\hline HB89 2344+092 & 15.96 & $\cdots$ & 14.99 & 13.55 & 0.677 & 4086.8 & 24.57 & 27.43 & $8,5,6$ \\
\hline
\end{tabular}

Note. - Columns list the source name, optical and near-IR magnitudes, luminosity distance calculated according to Wright (2006), specific UV and $21 \mathrm{~cm}$ radio luminosity in the host galaxy reference frame, and references for the magnitudes. For sources with only one magnitude listed, we do not calculate a UV luminosity.

Optical band magnitudes references: 1 - Stanghellini et al. (1993); 2 - Healey et al. (2008); 3- Stickel \& Kuehr (1996); $4-$ Wills \& Lynds (1978); 5 - Healey et al. (2008); 6 - 2MASS; Skrutskie et al. (2006); 7 - Snellen et al. (2002); 8 - SuperCOSMOS Sky Survey; Hambly et al. (2001); 9 Sandage (1965); 10 - Atlee \& Gould (2007); 11 - Kuhn (2004); 12 -Elvis et al. (1994); 13 - Francis et al. (2000); 14 - Kellermann et al. (1998);

15-Odell et al. (1978a); 16 - O'Dell et al. (1978); 17-Glass (1981); 18 - O'Dea et al. (1991); 19 - Sbarufatti et al. (2005); 20 - McAlary et al. (1983); 21 - Godwin et al. (1977); 22 - Gardner \& Whiteoak (1978); 23 - Wright, Ables \& Allen (1983); 24-Schmitt \& Kinney (1996); 25Drinkwater et al. (1997); 26 - Cody \& Braun (2003); 27 - Curran \& Whiting (2010); 28 - Sandage et al. (1965a); 29 - de Vries et al. (1997b); 30 Ellison, Hall, \& Lira (2005); 31 - Lister \& Homan (2005); 32 - SDSS DR6; Adelman-McCarthy et al. (2008); 33 - Allen, Ward \& Hyland (1982);

34 - Ramírez et al. (2004); 35 - Hyland \& Allen (1982); 36 - Rao et al. (2006); 37 - Chun et al. (2006); 38 - Labiano et al. (2007); 39 - Sbarufatti et al. (2009); 40 - Chen et al. (2005); 41 -Simpson \& Rawlings (2000); 42 - Jorgenson et al. (2006); 43 - Carballo et al. (1999); 44 - Matthews \& Sandage (1963); 45 - Sandage \& Wyndham (1965b); 46 -Fiorucci, Ciprini, \& Tosti (2004); 47 - Tapia et al. (1976); 48 - Kotilainen et al. (2005); 49 - Ojha et al. (2009); 50 - de Vries et al. (2000); 51 - Smith \& Heckman (1989); 52 - Drake et al. (2004); 53 - de Vries et al. (1998).

\section{APPENDIX}

We show the normalized spectra for the 102 RFI-free sources searched for intrinsic HI $21 \mathrm{~cm}$ (Figure 14) and intrinsic $\mathrm{OH} 18 \mathrm{~cm}$ absorption in sources with known $\mathrm{OH} 21 \mathrm{~cm}$ absorption (Figure 15). The observable velocity span for each source is a few thousand $\mathrm{km} \mathrm{s}^{-1}$ above/below with respect to the systemic velocity of the host galaxy and is typically determined by the RFI conditions rather than the spectral bandwidth. Table 2 lists the measured upper limits to the column densities for the $21 \mathrm{~cm}$ absorption line search. Table 3 lists the upper limits for the OH absorption line search. 

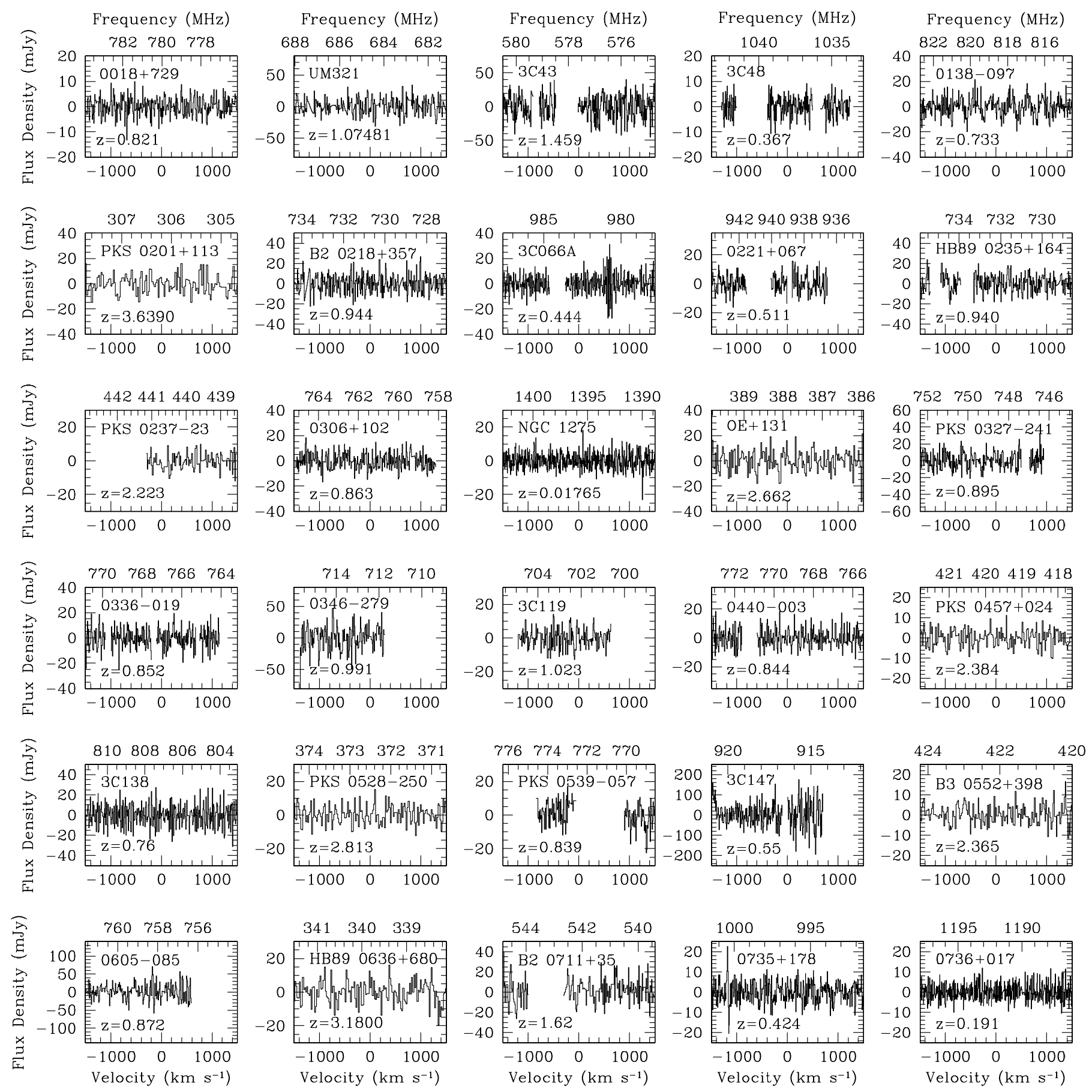

Figure 14. RFI-free sources not detected in HI $21 \mathrm{~cm}$ absorption. The velocity scale is in the rest frame of each object, defined by the heliocentric optical redshift of each radio source (Table 1). Spectral regions lost to radio frequency interference are not plotted. Each spectrum spans $\pm 1500 \mathrm{~km} \mathrm{~s}^{-1}$ except for PKS $0742+10$, where the x-axis has been shifted to show the feature that arises at $z=2.64$. The upper spectrum in PKS 0742+10 shows the Curran et al. (2013) data, supporting our non-detection interpretation of the possible absorption feature. 

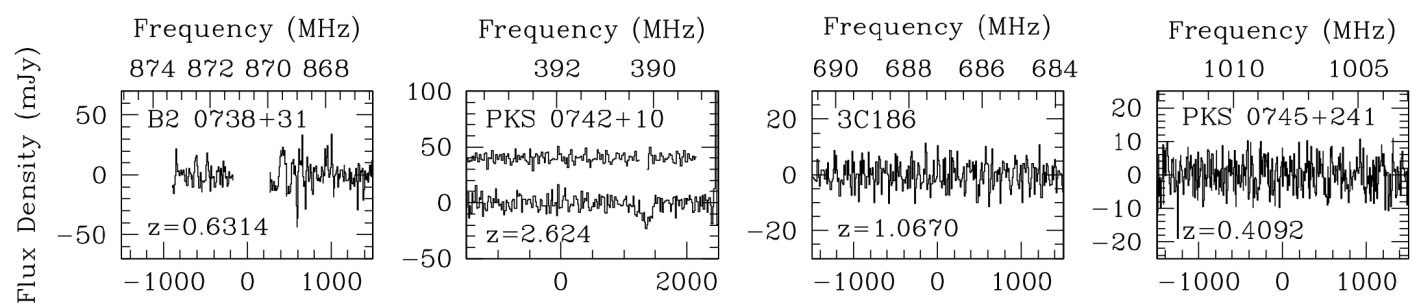

Frequency (MHz)
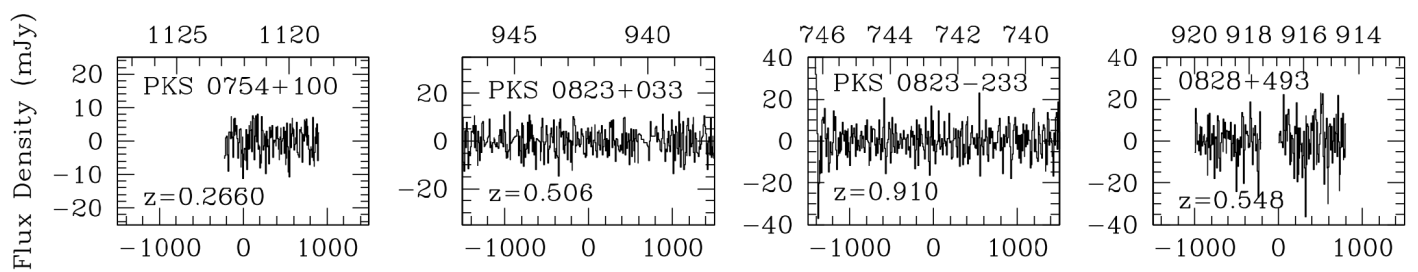

$\begin{array}{llll}311 & 310 & 309 & 308\end{array}$

20 E CoINS $\mathrm{F} 0753+4231]$

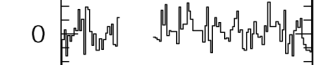

$-20 E-z=3.5892$

$\begin{array}{lll}-1000 & 0 & 1000\end{array}$
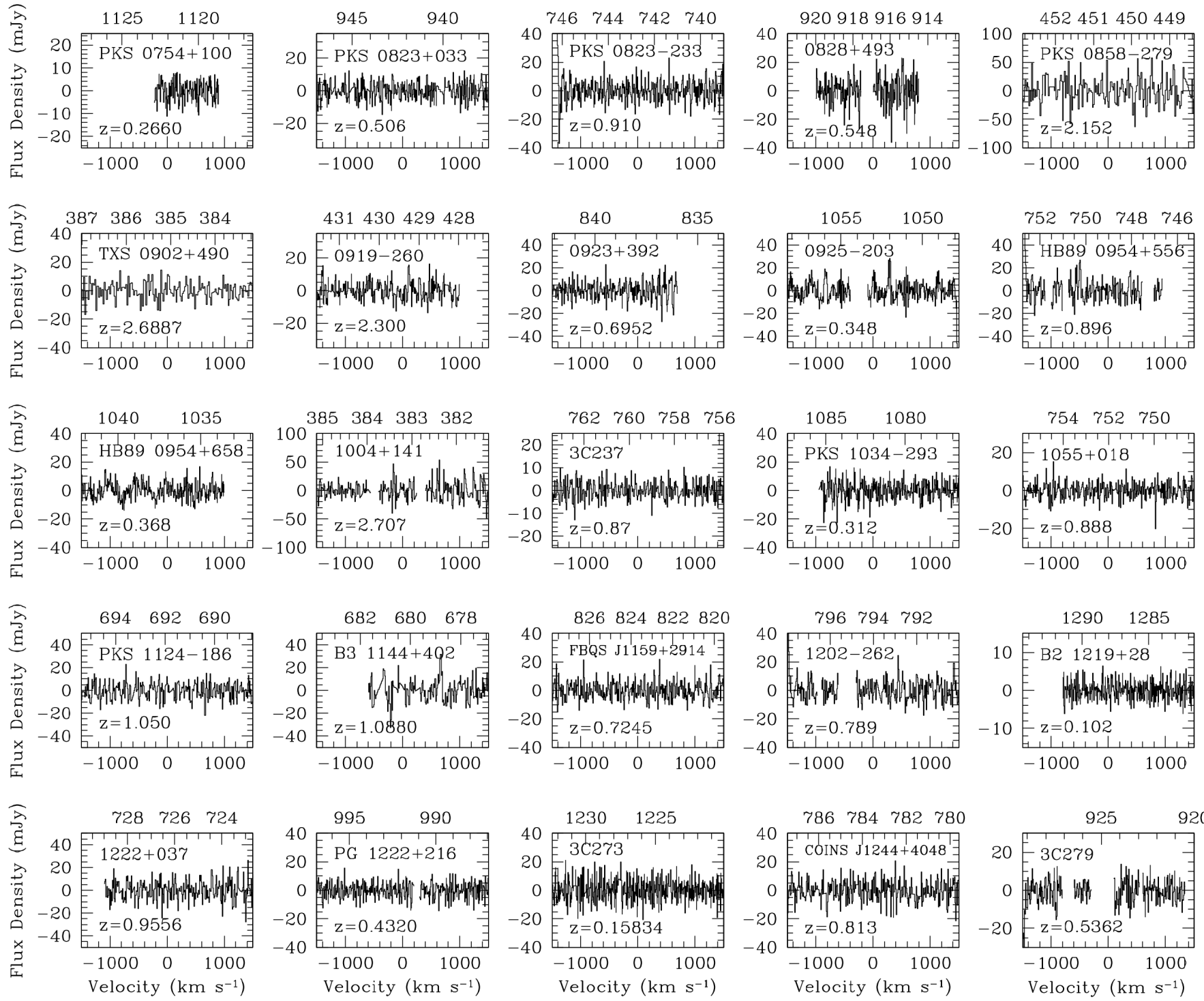

Figure 14. con't. 

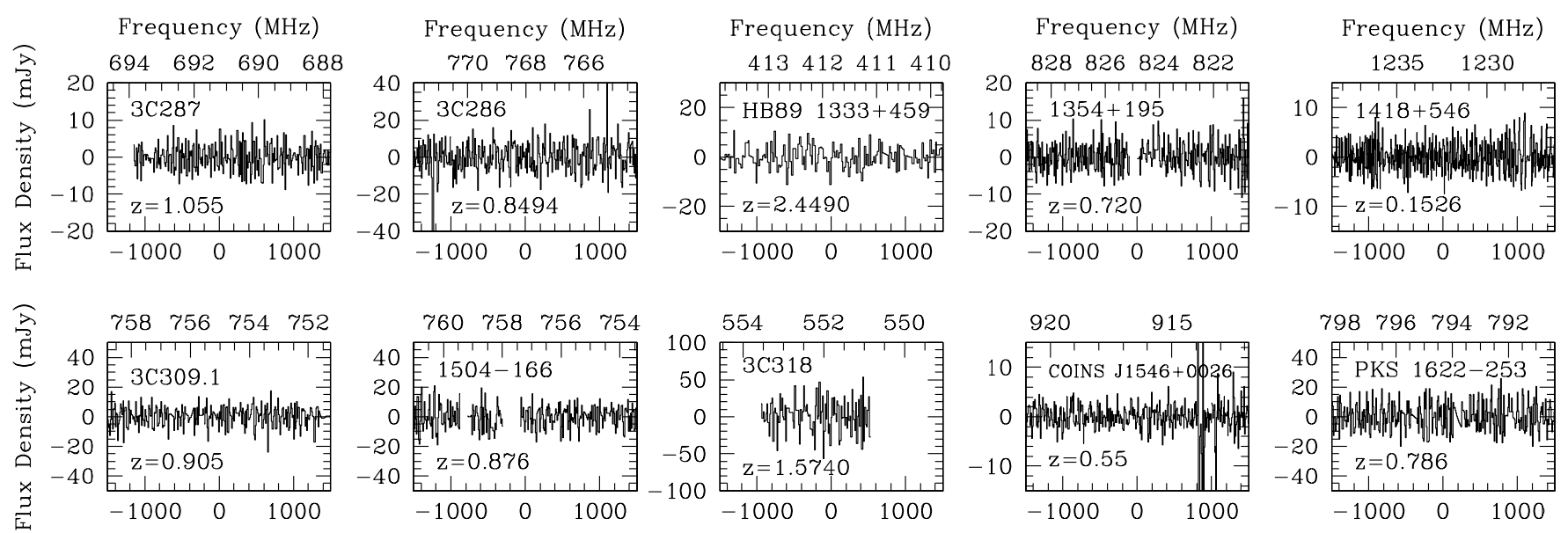

798796794792
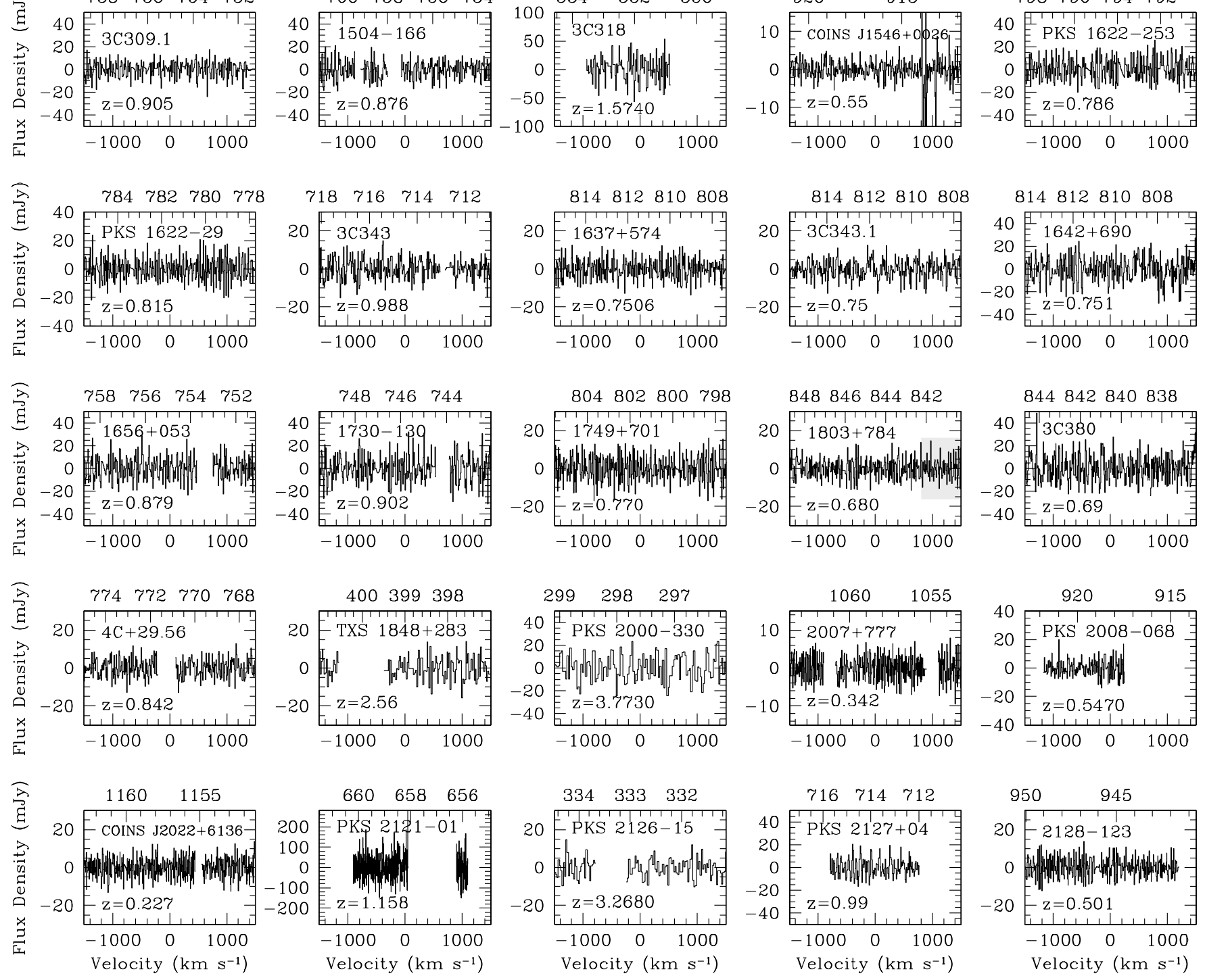

Figure 14. con't. 

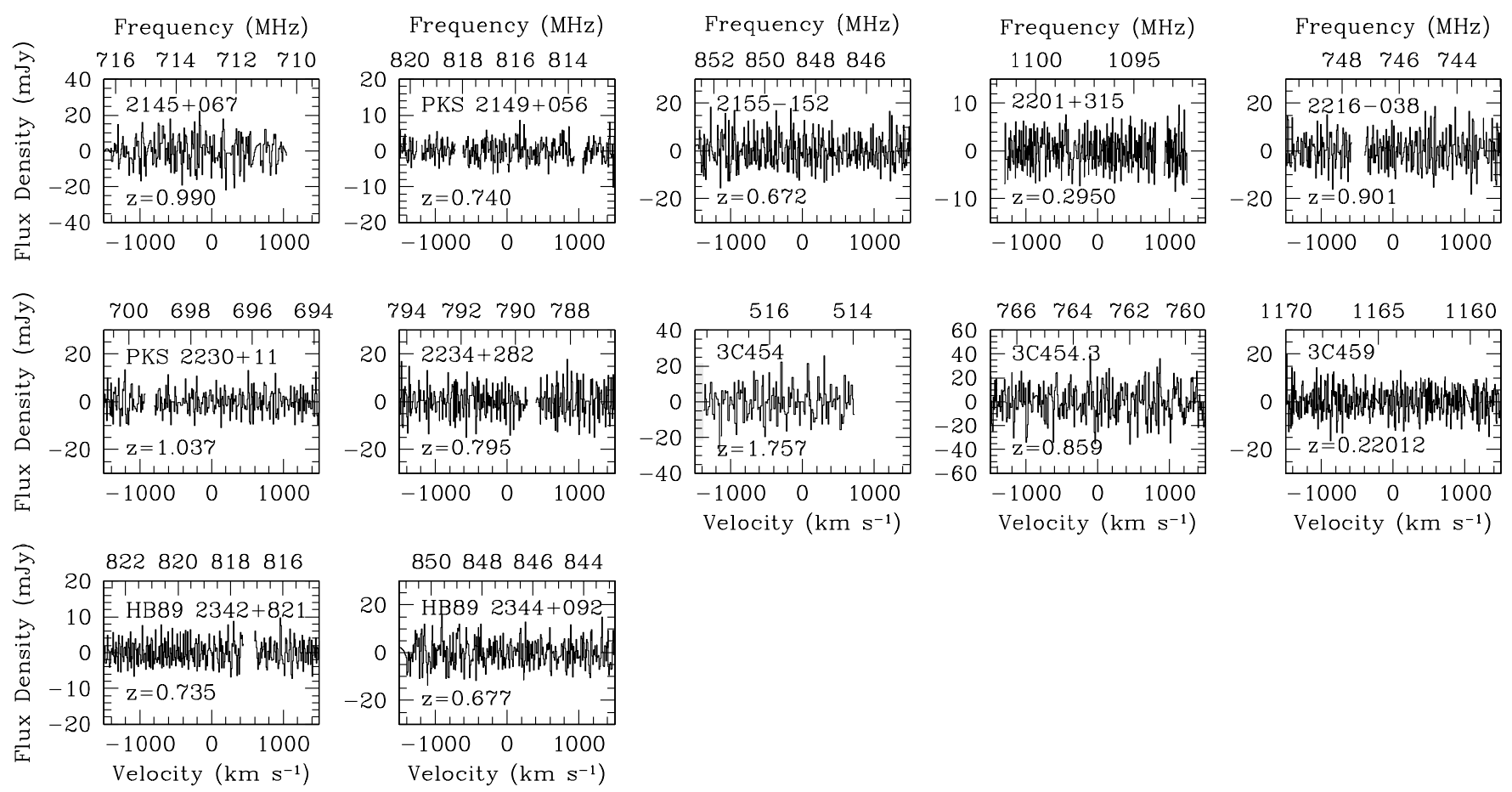

Figure 14. con't.
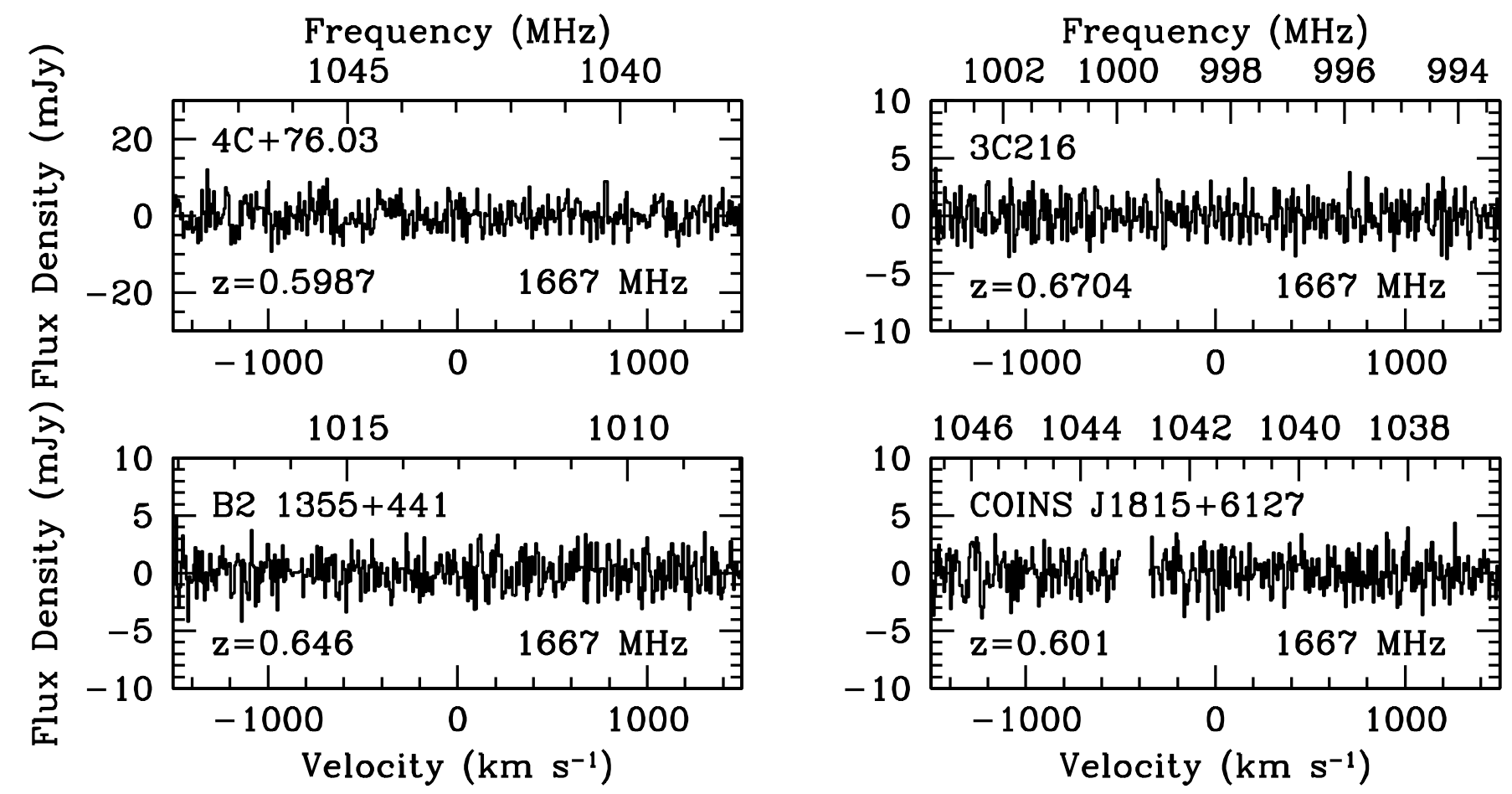

Figure 15. OH $1667 \mathrm{MHz}$ spectra of the four RFI-free intrinsic HI $21 \mathrm{~cm}$ absorption line systems searched for OH. The velocity scale is in the rest-frame of each source, defined by the redshift of the $21 \mathrm{~cm}$ absorber. Spectral regions lost to radio frequency interference are omitted. 